JOURNAL OF THE

AMERICAN MATHEMATICAL SOCIETY

Volume 19, Number 2, Pages 385-446

S 0894-0347(05)00513-8

Article electronically published on December 7, 2005

\title{
SHRINKWRAPPING AND THE TAMING OF HYPERBOLIC 3-MANIFOLDS
}

\author{
DANNY CALEGARI AND DAVID GABAI
}

\section{INTRODUCTION}

During the period 1960-1980, Ahlfors, Bers, Kra, Marden, Maskit, Sullivan, Thurston and many others developed the theory of geometrically finite ends of hyperbolic 3-manifolds. It remained to understand those ends which are not geometrically finite; such ends are called geometrically infinite.

Around 1978 William Thurston gave a conjectural description of geometrically infinite ends of complete hyperbolic 3-manifolds. An example of a geometrically infinite end is given by an infinite cyclic covering space of a closed hyperbolic 3manifold which fibers over the circle. Such an end has cross sections of uniformly bounded area. By contrast, the area of sections of geometrically finite ends grows exponentially in the distance from the convex core.

For the sake of clarity we will assume throughout this introduction that $N=$ $\mathbb{H}^{3} / \Gamma$ where $\Gamma$ is parabolic free. Precise statements of the parabolic case will be given in $\S 7$.

Thurston's idea was formalized by Bonahon $\mathrm{BO}$ and Canary $\mathrm{Ca}$ with the following.

Definition 0.1. An end $\mathcal{E}$ of a hyperbolic 3-manifold $N$ is simply degenerate if it has a closed neighborhood of the form $S \times[0, \infty)$ where $S$ is a closed surface, and there exists a sequence $\left\{S_{i}\right\}$ of $\mathrm{CAT}(-1)$ surfaces exiting $\mathcal{E}$ which are homotopic to $S \times 0$ in $\mathcal{E}$. This means that there exists a sequence of maps $f_{i}: S \rightarrow N$ such that the induced path metrics induce CAT(-1) structures on the $S_{i}$ 's, $f\left(S_{i}\right) \subset S \times[i, \infty)$ and $f$ is homotopic to a homeomorphism onto $S \times 0$ via a homotopy supported in $S \times[0, \infty)$.

Here by CAT(-1), we mean as usual a geodesic metric space for which geodesic triangles are "thinner" than comparison triangles in hyperbolic space. If the metrics pulled back by the $f_{i}$ are smooth, this is equivalent to the condition that the Riemannian curvature is bounded above by -1 . See $[\mathrm{BH}]$ for a reference. Note that by Gauss-Bonnet, the area of a $\operatorname{CAT}(-1)$ surface can be estimated from its Euler characteristic; it follows that a simply degenerate end has cross sections of uniformly bounded area, just like the end of a cyclic cover of a manifold fibering over the circle.

Received by the editors June 22, 2004.

2000 Mathematics Subject Classification. Primary 57M50, 57N10; Secondary 30F40, 49F10.

The first author was partially supported by Therese Calegari and NSF grant DMS-0405491.

The second author was partially supported by NSF grant DMS-0071852.

(C) 2005 by Danny Calegari and David Gabai 
Francis Bonahon $[\mathrm{Bo}$ observed that geometrically infinite ends are exactly those ends possessing an exiting sequence of closed geodesics. This will be our working definition of such ends throughout this paper.

The following is our main result.

Theorem 0.2. An end $\mathcal{E}$ of a complete hyperbolic 3-manifold $N$ with finitely generated fundamental group is simply degenerate if there exists a sequence of closed geodesics exiting $\mathcal{E}$.

Consequently we have:

Theorem 0.3. Let $N$ be a complete hyperbolic 3-manifold with finitely generated fundamental group. Then every end of $N$ is geometrically tame; i.e., it is either geometrically finite or simply degenerate.

In 1974 Marden $\mathrm{Ma}$ showed that a geometrically finite hyperbolic 3-manifold is topologically tame, i.e., is the interior of a compact 3-manifold. He asked whether all complete hyperbolic 3-manifolds with finitely generated fundamental group are topologically tame. This question is now known as the Tame Ends Conjecture or Marden Conjecture.

Theorem 0.4. If $N$ is a complete hyperbolic 3-manifold with finitely generated fundamental group, then $N$ is topologically tame.

Ian Agol $\mathrm{Ag}$ has independently proven Theorem 0.4 .

There have been many important steps towards Theorem 0.2 The seminal result was obtained by Thurston ([T], Theorem 9.2) who proved Theorems 0.3 and 0.4 for certain algebraic limits of quasi-Fuchsian groups. Bonahon [Bo] established Theorems 0.2 and 0.4 when $\pi_{1}(N)$ is freely indecomposable, and Canary $\mathrm{Ca}$. proved that topological tameness implies geometrical tameness. Results in the direction of 0.4 were also obtained by Canary-Minsky CaM, Kleineidam-Souto [KS, Evans Ev], Brock-Bromberg-Evans-Souto BBES, Ohshika Oh], Brock-Souto [BS] and Souto [So].

Thurston first discovered how to obtain analytic conclusions from the existence of exiting sequences of CAT $(-1)$ surfaces. Thurston's work as generalized by Bonahon Bo and Canary $\mathrm{Ca}$ combined with Theorem 0.2 yields a positive proof of the Ahlfors' Measure Conjecture A2.

Theorem 0.5. If $\Gamma$ is a finitely generated Kleinian group, then the limit set $L_{\Gamma}$ is either $S_{\infty}^{2}$ or has Lebesgue measure zero. If $L_{\Gamma}=S_{\infty}^{2}$, then $\Gamma$ acts ergodically on $S_{\infty}^{2}$.

Theorem 0.5 is one of the many analytical consequences of our main result. Indeed, Theorem 0.2 implies that a complete hyperbolic 3-manifold $N$ with finitely generated fundamental group is analytically tame as defined by Canary [Ca]. It follows from Canary that the various results of [Ca, §9] hold for $N$.

Our main result is the last step needed to prove the following monumental result, the other parts being established by Alhfors, Bers, Kra, Marden, Maskit, Mostow, Prasad, Sullivan, Thurston, Minsky, Masur-Minsky, Brock-Canary-Minsky, Ohshika, Kleineidam-Souto, Lecuire, Kim-Lecuire-Ohshika, Hossein-Souto and Rees. See [Mi] and $\mathrm{BCM}$.

Theorem 0.6 (Classification Theorem). If $N$ is a complete hyperbolic 3-manifold with finitely generated fundamental group, then $N$ is determined up to isometry by 
its topological type, the conformal boundary of its geometrically finite ends and the ending laminations of its geometrically infinite ends.

The following result was conjectured by Bers, Sullivan and Thurston. Theorem 0.4 is one of many results, many of them recent, needed to build a proof. Major contributions were made by Alhfors, Bers, Kra, Marden, Maskit, Mostow, Prasad, Sullivan, Thurston, Minsky, Masur-Minsky, Brock-Canary-Minsky, Ohshika, Kleineidam-Souto, Lecuire, Kim-Lecuire-Ohshika, Hossein-Souto, Rees, Bromberg and Brock-Bromberg.

Theorem 0.7 (Density Theorem). If $N=\mathbb{H}^{3} / \Gamma$ is a complete hyperbolic 3manifold with finitely generated fundamental group, then $\Gamma$ is the algebraic limit of geometrically finite Kleinian groups.

The main technical innovation of this paper is a new technique called shrinkwrapping for producing CAT(-1) surfaces in hyperbolic 3-manifolds. Historically, such surfaces have been immensely important in the study of hyperbolic 3-manifolds; e.g., see [T], Bo, $\mathrm{Ca}$ and $\mathrm{CaM}]$.

Given a locally finite set $\Delta$ of pairwise disjoint simple closed curves in the 3manifold $N$, we say that the embedded surface $S \subset N$ is 2-incompressible rel. $\Delta$ if every compressing disc for $S$ meets $\Delta$ at least twice. Here is a sample theorem.

Theorem 0.8 (Existence of shrinkwrapped surface). Let $M$ be a complete, orientable, parabolic free hyperbolic 3-manifold, and let $\Gamma$ be a finite collection of pairwise disjoint simple closed geodesics in $M$. Furthermore, let $S \subset M \backslash \Gamma$ be a closed embedded 2-incompressible surface rel. $\Gamma$ which is either nonseparating in $M$ or separates some component of $\Gamma$ from another. Then $S$ is homotopic to a $C A T(-1)$ surface $T$ via a homotopy

$$
F: S \times[0,1] \rightarrow M
$$

such that

(1) $F(S \times 0)=S$,

(2) $F(S \times t)=S_{t}$ is an embedding disjoint from $\Gamma$ for $0 \leq t<1$,

(3) $F(S \times 1)=T$,

(4) If $T^{\prime}$ is any other surface with these properties, then area $(T) \leq \operatorname{area}\left(T^{\prime}\right)$.

We say that $T$ is obtained from $S$ by shrinkwrapping rel. $\Gamma$, or if $\Gamma$ is understood, $T$ is obtained from $S$ by shrinkwrapping.

In fact, we prove the stronger result that $T$ is $\Gamma$-minimal (to be defined in $\S 1$ ), which implies in particular that it is intrinsically $\mathrm{CAT}(-1)$.

Here is the main technical result of this paper.

Theorem 0.9. Let $\mathcal{E}$ be an end of the complete orientable hyperbolic 3-manifold $N$ with finitely generated fundamental group. Let $C$ be a 3-dimensional compact core of $N, \partial_{\mathcal{E}} C$ the component of $\partial C$ facing $\mathcal{E}$ and $g=\operatorname{genus}\left(\partial_{\mathcal{E}} C\right)$. If there exists a sequence of closed geodesics exiting $\mathcal{E}$, then there exists a sequence $\left\{S_{i}\right\}$ of $C A T(-1)$ surfaces of genus $g$ exiting $\mathcal{E}$ such that each $S_{i}$ is homologically separating in $\mathcal{E}$. That is, each $S_{i}$ homologically separates $\partial_{\mathcal{E}} C$ from $\mathcal{E}$.

Theorem 0.4 can now be deduced from Theorem 0.9 and Souto $[\mathrm{So}$; however, we prove that Theorem 0.9 implies Theorem 0.4 using only 3-manifold topology and elementary hyperbolic geometry. 
The proof of Theorem 0.9 blends elementary aspects of minimal surface theory, hyperbolic geometry, and 3-manifold topology. The method will be demonstrated in $\S 4$ where we give a proof of Canary's theorem. The first-time reader is urged to begin with that section.

This paper is organized as follows. In $\S 1$ and $\S 2$ we establish the shrinkwrapping technique for finding $\mathrm{CAT}(-1)$ surfaces in hyperbolic 3-manifolds. In $\S 3$ we prove the existence of $\epsilon$-separated simple geodesics exiting the end of parabolic free manifolds. In $\S 4$ we prove Canary's theorem. This proof will model the proof of the general case. The general strategy will be outlined at the end of that section. In $\S 5$ we develop the topological theory of end reductions in 3-manifolds. In $\S 6$ we give the proofs of our main results. In $\S 7$ we give the necessary embellishments of our methods to state and prove our results in the case of manifolds with parabolic cusps.

Notation 0.10. If $X \subset Y$, then $N(X)$ denotes a regular neighborhood of $X$ in $Y$ and $\operatorname{int}(X)$ denotes the interior of $X$. If $X$ is a topological space, then $|X|$ denotes the number of components of $X$. If $A, B$ are topological subspaces of a third space, then $A \backslash B$ denotes the intersection of $A$ with the complement of $B$.

\section{SHRINKWRAPPING}

In this section, we introduce a new technical tool for finding CAT $(-1)$ surfaces in hyperbolic 3-manifolds, called shrinkwrapping. Roughly speaking, given a collection of simple closed geodesics $\Gamma$ in a hyperbolic 3-manifold $M$ and an embedded surface $S \subset M \backslash \Gamma$, a surface $T \subset M$ is obtained from $S$ by shrinkwrapping $S$ rel. $\Gamma$ if it is homotopic to $S$, can be approximated by an isotopy from $S$ supported in $M \backslash \Gamma$, and is the least area subject to these constraints.

Given mild topological conditions on $M, \Gamma, S$ (namely 2-incompressibility, to be defined below) the shrinkwrapped surface exists, and is CAT $(-1)$ with respect to the path metric induced by the Riemannian metric on $M$.

We use some basic analytical tools throughout this section, including the GaussBonnet formula, the coarea formula, and the Arzela-Ascoli theorem. At a number of points we must invoke results from the literature to establish existence of minimal surfaces ( $\underline{\mathrm{MSY}}]$ ), existence of limits with area and curvature control ([iSc $)$, and regularity of the shrinkwrapped surfaces along $\Gamma$ ([Ri], Fre $)$. General references are $\mathrm{CM}$, Js], Fed and $\mathrm{B}$.

1.1. Geometry of surfaces. For convenience, we state some elementary but fundamental lemmas concerning curvature of (smooth) surfaces in Riemannian 3manifolds.

We use the following standard terms to refer to different kinds of minimal surfaces:

Definition 1.1. A smooth surface $\Sigma$ in a Riemannian 3-manifold is minimal if it is a critical point for area with respect to all smooth compactly supported variations. It is locally least area (also called stable) if it is a local minimum for area with respect to all smooth, compactly supported variations. A closed, embedded surface is globally least area if it is an absolute minimum for area amongst all smooth surfaces in its isotopy class.

Note that we do not require that our minimal or locally least area surfaces are complete. 
Any subsurface of a globally least area surface is locally least area, and a locally least area surface is minimal. A smooth surface is minimal iff its mean curvature vector field vanishes identically. For more details, consult [CM], especially chapter 5.

The intrinsic curvature of a minimal surface is controlled by the geometry of the ambient manifold. The following lemma is formula 5.6 on page 100 of [CM].

Lemma 1.2 (Monotonicity of curvature). Let $\Sigma$ be a minimal surface in a Riemannian manifold $M$. Let $K_{\Sigma}$ denote the curvature of $\Sigma$, and $K_{M}$ the sectional curvature of $M$. Then restricted to the tangent space $T \Sigma$,

$$
K_{\Sigma}=K_{M}-\frac{1}{2}|A|^{2}
$$

where $A$ denotes the second fundamental form of $\Sigma$.

In particular, if the Riemannian curvature on $M$ is bounded from above by some constant $K$, then the curvature of a minimal surface $\Sigma$ in $M$ is also bounded above by $K$.

The following lemma is just the usual Gauss-Bonnet formula:

Lemma 1.3 (Gauss-Bonnet formula). Let $\Sigma$ be a $C^{3}$ Riemannian surface with (possibly empty) $C^{3}$ boundary $\partial \Sigma$. Let $K_{\Sigma}$ denote the Gauss curvature of $\Sigma$, and $\kappa$ the geodesic curvature along $\partial \Sigma$. Then

$$
\int_{\Sigma} K_{\Sigma}=2 \pi \chi(\Sigma)-\int_{\partial \Sigma} \kappa d l
$$

Many simple proofs exist in the literature. For example, see [Js.

If $\partial \Sigma$ is merely piecewise $C^{3}$, with finitely many corners $p_{i}$ and external angles $\alpha_{i}$, the Gauss-Bonnet formula must be modified as follows:

Lemma 1.4 (Gauss-Bonnet with corners). Let $\Sigma$ be a $C^{3}$ Riemannian surface with boundary $\partial \Sigma$ which is piecewise $C^{3}$ and has external angles $\alpha_{i}$ at finitely many points $p_{i}$. Let $K_{\Sigma}$ and $\kappa$ be as above. Then

$$
\int_{\Sigma} K_{\Sigma}=2 \pi \chi(\Sigma)-\int_{\partial \Sigma} \kappa d l-\sum_{i} \alpha_{i}
$$

Observe for $a b c$ a geodesic triangle with external angles $\alpha_{1}, \alpha_{2}, \alpha_{3}$ that Lemma 1.4 implies

$$
\int_{a b c} K=2 \pi-\sum_{i} \alpha_{i}
$$

Notice that the geodesic curvature $\kappa$ vanishes precisely when $\partial \Sigma$ is a geodesic, that is, a critical point for the length functional. More generally, let $\nu$ be the normal bundle of $\partial \Sigma$ in $\Sigma$, oriented so that the inward unit normal is a positive section. The exponential map restricted to $\nu$ defines a map

$$
\phi: \partial \Sigma \times[0, \epsilon] \rightarrow \Sigma
$$

for small $\epsilon$, where $\phi(\cdot, 0)=\left.\mathrm{Id}\right|_{\partial \Sigma}$, and $\phi(\partial \Sigma, t)$ for small $t$ is the boundary in $\Sigma$ of the tubular $t$ neighborhood of $\partial \Sigma$. Then

$$
\int_{\partial \Sigma} \kappa d l=-\left.\frac{d}{d t}\right|_{t=0} \operatorname{length}\left(\phi_{t}(\partial \Sigma)\right)
$$


Note that if $\Sigma$ is a surface with sectional curvature bounded above by -1 , then by integrating this formula we see that the ball $B_{t}(p)$ of radius $t$ in $\Sigma$ about a point $p \in \Sigma$ satisfies

$$
\operatorname{area}\left(B_{t}(p)\right) \geq 2 \pi(\cosh (t)-1)>\pi t^{2}
$$

for small $t>0$.

1.2. Comparison geometry. For basic elements of the theory of comparison geometry, see $[\mathrm{BH}]$.

Definition 1.5 (Comparison triangle). Let $a_{1} a_{2} a_{3}$ be a geodesic triangle in a geodesic metric space $X$. Let $\kappa \in \mathbb{R}$ be given. A $\kappa$-comparison triangle is a geodesic triangle $\overline{a_{1} a_{2} a_{3}}$ in the complete simply-connected Riemannian 2-manifold of constant sectional curvature $\kappa$, where the edges $a_{i} a_{j}$ and $\overline{a_{i} a_{j}}$ satisfy

$$
\text { length }\left(a_{i} a_{j}\right)=\operatorname{length}\left(\overline{a_{i} a_{j}}\right) \text {. }
$$

Given a point $x \in a_{1} a_{2}$ on one of the edges of $a_{1} a_{2} a_{3}$, there is a corresponding point $\bar{x} \in \overline{a_{1} a_{2}}$ on one of the edges of the comparison triangle, satisfying

$$
\operatorname{length}\left(a_{1} x\right)=\operatorname{length}\left(\overline{a_{1} x}\right)
$$

and

$$
\text { length }\left(x a_{2}\right)=\operatorname{length}\left(\overline{x a_{2}}\right) .
$$

Remark 1.6. Note that if $\kappa>0$, the comparison triangle might not exist if the edge lengths are too big, but if $\kappa \leq 0$ the comparison triangle always exists and is unique up to isometry.

There is a slight issue of terminology to be aware of here. In a surface, a triangle is a polygonal disk with 3 geodesic edges. In a path metric space, a triangle is just a union of 3 geodesic segments with common endpoints.

Definition $1.7(\operatorname{CAT}(\kappa))$. Let $S$ be a closed surface with a path metric $g$. Let $\tilde{S}$ denote the universal cover of $S$, with path metric induced by the pullback of the path metric $g$. Let $\kappa \in \mathbb{R}$ be given. $S$ is said to be $C A T(\kappa)$ if for every geodesic triangle $a b c$ in $\tilde{S}$, and every point $z$ on the edge $b c$, the distance in $\tilde{S}$ from $a$ to $z$ is no more than the distance from $\bar{a}$ to $\bar{z}$ in a $\kappa$-comparison triangle.

By Lemma 1.4 applied to geodesic triangles, one can show that a $C^{3}$ surface $\Sigma$ with sectional curvature $K_{\Sigma}$ satisfying $K_{\Sigma} \leq \kappa$ everywhere is $\operatorname{CAT}(\kappa)$ with respect to the Riemannian path metric. This fact is essentially due to Alexandrov; see B] for a proof.

More generally, suppose $\Sigma$ is a surface which is $C^{3}$ outside a closed, nowhere dense subset $X \subset \Sigma$. Furthermore, suppose that $K_{\Sigma} \leq \kappa$ holds in $\Sigma \backslash X$, and suppose that the formula from Lemma 1.4 holds for every geodesic triangle with vertices in $\Sigma \backslash X$ (which is a dense set of geodesic triangles). Then the same argument shows that $\Sigma$ is $\operatorname{CAT}(\kappa)$. See, e.g., [Re, $\S 8$, pp. 135-140] for more details and a general discussion of metric surfaces with (integral) curvature bounds.

Definition 1.8 ( $\Gamma$-minimal surfaces). Let $\kappa \in \mathbb{R}$ be given. Let $M$ be a complete Riemannian 3-manifold with sectional curvature bounded above by $\kappa$, and let $\Gamma$ be an embedded collection of simple closed geodesics in $M$. An immersion

$$
\psi: S \rightarrow M
$$


is $\Gamma$-minimal if it is smooth with mean curvature 0 in $M \backslash \Gamma$ and is metrically $\mathrm{CAT}(\kappa)$ with respect to the path metric induced by $\psi$ from the Riemannian metric on $M$.

Notice by Lemma 1.2 that a smooth surface $S$ with mean curvature 0 in $M$ is $\operatorname{CAT}(\kappa)$, so a minimal surface (in the usual sense) is an example of a $\Gamma$-minimal surface.

\subsection{Statement of shrinkwrapping theorem.}

Definition 1.9 (2-incompressibility). An embedded surface $S$ in a 3-manifold $M$ disjoint from a collection $\Gamma$ of simple closed curves is said to be 2-incompressible rel. $\Gamma$ if any essential compressing disk for $S$ must intersect $\Gamma$ in at least two points. If $\Gamma$ is understood, we say $S$ is 2-incompressible.

Theorem 1.10 (Existence of shrinkwrapped surface). Let $M$ be a complete, orientable, parabolic free hyperbolic 3-manifold, and let $\Gamma$ be a finite collection of pairwise disjoint simple closed geodesics in $M$. Furthermore, let $S \subset M \backslash \Gamma$ be a closed embedded 2-incompressible surface rel. $\Gamma$ which is either nonseparating in $M$ or separates some component of $\Gamma$ from another. Then $S$ is homotopic to a $\Gamma$-minimal surface $T$ via a homotopy

$$
F: S \times[0,1] \rightarrow M
$$

such that

(1) $F(S \times 0)=S$,

(2) $F(S \times t)=S_{t}$ is an embedding disjoint from $\Gamma$ for $0 \leq t<1$,

(3) $F(S \times 1)=T$,

(4) if $T^{\prime}$ is any other surface with these properties, then area $(T) \leq \operatorname{area}\left(T^{\prime}\right)$.

We say that $T$ is obtained from $S$ by shrinkwrapping rel. $\Gamma$, or if $\Gamma$ is understood, $T$ is obtained from $S$ by shrinkwrapping.

The remainder of this section will be taken up with the proof of Theorem 1.10.

Remark 1.11. In fact, for our applications, the property we want to use of our surface $T$ is that we can estimate its diameter (rel. the thin part of $M$ ) from its Euler characteristic. This follows from a Gauss-Bonnet estimate and the bounded diameter lemma (Lemma 1.15, to be proved below). In fact, our argument will show directly that the surface $T$ satisfies Gauss-Bonnet; the fact that it is CAT $(-1)$ is logically superfluous for the purposes of this paper.

\subsection{Deforming metrics along geodesics.}

Definition 1.12 ( $\delta$-separation). Let $\Gamma$ be a collection of disjoint simple geodesics in a Riemannian manifold $M$. The collection $\Gamma$ is $\delta$-separated if any path $\alpha: I \rightarrow M$ with endpoints on $\Gamma$ and satisfying

$$
\text { length }(\alpha(I)) \leq \delta
$$

is homotopic rel. endpoints into $\Gamma$. The supremum of such $\delta$ is called the separation constant of $\Gamma$. The collection $\Gamma$ is weakly $\delta$-separated if

$$
\operatorname{dist}\left(\gamma, \gamma^{\prime}\right)>\delta
$$

whenever $\gamma, \gamma^{\prime}$ are distinct components of $\Gamma$. The supremum of such $\delta$ is called the weak separation constant of $\Gamma$. 
Definition 1.13 (Neighborhood and tube neighborhood). Let $r>0$ be given. For a point $x \in M$, we let $N_{r}(x)$ denote the closed ball of radius $r$ about $x$, and let $N_{<r}(x), \partial N_{r}(x)$ denote, respectively, the interior and the boundary of $N_{r}(x)$. For a closed geodesic $\gamma$ in $M$, we let $N_{r}(\gamma)$ denote the closed tube of radius $r$ about $\gamma$, and let $N_{<r}(\gamma), \partial N_{r}(\gamma)$ denote, respectively, the interior and the boundary of $N_{r}(\gamma)$. If $\Gamma$ denotes a union of geodesics $\gamma_{i}$, then we use the shorthand notation

$$
N_{r}(\Gamma)=\bigcup_{\gamma_{i}} N_{r}\left(\gamma_{i}\right)
$$

Remark 1.14. Topologically, $\partial N_{r}(x)$ is a sphere and $\partial N_{r}(\gamma)$ is a torus, for sufficiently small $r$. Similarly, $N_{r}(x)$ is a closed ball, and $N_{r}(\gamma)$ is a closed solid torus. If $\Gamma$ is $\delta$-separated, then $N_{\delta / 2}(\Gamma)$ is a union of solid tori.

Lemma 1.15 (Bounded Diameter Lemma). Let $M$ be a complete hyperbolic 3manifold. Let $\Gamma$ be a disjoint collection of $\delta$-separated embedded geodesics. Let $\epsilon>0$ be a Margulis constant for dimension 3 , and let $M_{\leq \epsilon}$ denote the subset of $M$ where the injectivity radius is at most $\epsilon$. If $S \subset M \backslash \Gamma$ is a 2-incompressible $\Gamma$-minimal surface, then there is a constant $C=C(\chi(S), \epsilon, \delta) \in \mathbb{R}$ and $n=n(\chi(S), \epsilon, \delta) \in \mathbb{Z}$ such that for each component $S_{i}$ of $S \cap\left(M \backslash M_{\leq \epsilon}\right)$, we have

$$
\operatorname{diam}\left(S_{i}\right) \leq C .
$$

Furthermore, $S$ can only intersect at most $n$ components of $M_{\leq \epsilon}$.

Proof. Since $S$ is 2-incompressible, any point $x \in S$ either lies in $M_{\leq \epsilon}$ or is the center of an embedded $m$-disk in $S$, where

$$
m=\min (\epsilon / 2, \delta / 2) \text {. }
$$

Since $S$ is CAT $(-1)$, Gauss-Bonnet implies that the area of an embedded $m$-disk in $S$ has area at least $2 \pi(\cosh (m)-1)>\pi m^{2}$.

This implies that if $x \in S \cap M \backslash M_{\leq \epsilon}$, then

$$
\operatorname{area}\left(S \cap N_{m}(x)\right) \geq \pi m^{2} .
$$

The proof now follows by a standard covering argument.

A surface $S$ satisfying the conclusion of the Bounded Diameter Lemma is sometimes said to have diameter bounded by $C$ modulo $M_{\leq \epsilon}$.

Remark 1.16. Note that if $\epsilon$ is a Margulis constant, then $M_{\leq \epsilon}$ consists of Margulis tubes and cusps. Note that the same argument shows that, away from the thin part of $M$ and an $\epsilon$-neighborhood of $\Gamma$, the diameter of $S$ can be bounded by a constant depending only on $\chi(S)$ and $\epsilon$.

The basic idea in the proof of Theorem 1.10 is to search for a least area representative of the isotopy class of the surface $S$, subject to the constraint that the track of this isotopy does not cross $\Gamma$. Unfortunately, $M \backslash \Gamma$ is not complete, so the prospects for doing minimal surface theory in this manifold are remote. To remedy this, we deform the metric on $M$ in a neighborhood of $\Gamma$ in such a way that we can guarantee the existence of a least area surface representative with respect to the deformed metric and then take a limit of such surfaces under a sequence of smaller and smaller such metric deformations. We describe the deformations of interest below. 
In fact, for technical reasons which will become apparent in $\$ 1.8$, the deformations described below are not quite adequate for our purposes, and we must consider metrics which are deformed twice - firstly, a mild deformation which satisfies curvature pinching $-1 \leq K \leq 0$, and which is totally Euclidean in a neighborhood of $\Gamma$, and secondly a deformation analogous to the kind described below in Definition 1.17, which is supported in this totally Euclidean neighborhood. Since the reason for this "double perturbation" will not be apparent until 1.8. we postpone discussion of such deformations until that time.

Definition 1.17 (Deforming metrics). Let $\delta>0$ be such that $\Gamma$ is $\delta$-separated. Choose some small $r$ with $r<\delta / 2$. For $t \in[0,1)$ we define a family of Riemannian metrics $g_{t}$ on $M$ in the following manner. The metrics $g_{t}$ agree with the hyperbolic metric away from some fixed tubular neighborhood $N_{r}(\Gamma)$.

Let

$$
h: N_{r(1-t)}(\Gamma) \rightarrow[0, r(1-t)]
$$

be the function whose value at a point $p$ is the hyperbolic distance from $p$ to $\Gamma$. We define a metric $g_{t}$ on $M$ which agrees with the hyperbolic metric outside $N_{r(1-t)}(\Gamma)$, and on $N_{r(1-t)}(\Gamma)$ is conformally equivalent to the hyperbolic metric, as follows. Let $\phi:[0,1] \rightarrow[0,1]$ be a $C^{\infty}$ bump function, which is equal to 1 on the interval $[1 / 3,2 / 3]$, which is equal to 0 on the intervals $[0,1 / 4]$ and $[3 / 4,1]$, and which is strictly increasing on $[1 / 4,1 / 3]$ and strictly decreasing on $[2 / 3,3 / 4]$. Then define the ratio

$$
\frac{g_{t} \text { length element }}{\text { hyperbolic length element }}=1+2 \phi\left(\frac{h(p)}{r(1-t)}\right) \text {. }
$$

We are really only interested in the behaviour of the metrics $g_{t}$ as $t \rightarrow 1$. As such, the choice of $r$ is irrelevant. However, for convenience, we will fix some small $r$ throughout the remainder of $\S 1$.

The deformed metrics $g_{t}$ have the following properties:

Lemma 1.18 (Metric properties). The $g_{t}$ metric satisfies the following properties:

(1) For each $t$ there is an $f(t)$ satisfying $r(1-t) / 4<f(t)<3 r(1-t) / 4$ such that the union of the tori $\partial N_{f(t)}(\Gamma)$ is totally geodesic for the $g_{t}$ metric.

(2) For each component $\gamma_{i}$ and each $t$, the metric $g_{t}$ restricted to $N_{r}\left(\gamma_{i}\right)$ admits a family of isometries which preserve $\gamma_{i}$ and acts transitively on the unit normal bundle (in $M$ ) to $\gamma_{i}$.

(3) The area of a disk cross section on $N_{r(1-t)}$ is $O\left((1-t)^{2}\right)$.

(4) The metric $g_{t}$ dominates the hyperbolic metric on 2-planes. That is, for all 2-vectors $\nu$, the $g_{t}$ area of $\nu$ is at least as large as the hyperbolic area of $\nu$.

Proof. Statement (2) follows from the fact that the definition of $g_{t}$ has the desired symmetries. Statements (3) and (4) follow from the fact that the ratio of the $g_{t}$ metric to the hyperbolic metric is pinched between 1 and 3. Now, a radially symmetric circle linking $\Gamma$ of radius $s$ has length $2 \pi \cosh (s)$ in the hyperbolic metric, and therefore has length

$$
2 \pi \cosh (s)\left(1+2 \phi\left(\frac{s}{r(1-t)}\right)\right)
$$

in the $g_{t}$ metric. For sufficiently small (but fixed) $r$, this function of $s$ has a local minimum on the interval $[r(1-t) / 4,3 r(1-t) / 4]$. It follows that the family of 
radially symmetric tori linking a component of $\Gamma$ has a local minimum for area in the interval $[r(1-t) / 4,3 r(1-t) / 4]$. By property $(2)$, such a torus must be totally geodesic for the $g_{t}$ metric.

Notation 1.19. We denote length of an arc $\alpha: I \rightarrow M$ with respect to the $g_{t}$ metric as length $(\alpha(I))$, and area of a surface $\psi: R \rightarrow M$ with respect to the $g_{t}$ metric as $\operatorname{area}_{t}(\psi(R))$.

1.5. Constructing the homotopy. As a first approximation, we wish to construct surfaces in $M \backslash \Gamma$ which are globally least area with respect to the $g_{t}$ metric. There are various tools for constructing least area surfaces in Riemannian 3 -manifolds under various conditions, and subject to various constraints. Typically one works in closed 3-manifolds, but if one wants to work in 3-manifolds with boundary, the "correct" boundary condition to impose is mean convexity. A cooriented surface in a Riemannian 3-manifold is said to be mean convex if the mean curvature vector of the surface always points to the negative side of the surface, where it does not vanish. Totally geodesic surfaces and other minimal surfaces are examples of mean convex surfaces, with respect to any co-orientation. Such surfaces act as barriers for minimal surfaces, in the following sense: suppose that $S_{1}$ is a mean convex surface and $S_{2}$ is a minimal surface. Suppose furthermore that $S_{2}$ is on the negative side of $S_{1}$. Then if $S_{2}$ and $S_{1}$ are tangent, they are equal. One should stress that this barrier property is local. See MSY for a more thorough discussion of barrier surfaces.

Lemma 1.20 (Minimal surface exists). Let $M, \Gamma, S$ be as in the statement of Theorem 1.10. Let $f(t)$ be as in Lemma 1.18, so that $\partial N_{f(t)}(\Gamma)$ is totally geodesic with respect to the $g_{t}$ metric. Then for each $t$, there exists an embedded surface $S_{t}$ isotopic in $M \backslash N_{f(t)}(\Gamma)$ to $S$, and which is globally $g_{t}$-least area among all such surfaces.

Proof. Note that with respect to the $g_{t}$ metrics, the surfaces $\partial N_{f(t)}(\Gamma)$ described in Lemma 1.18 are totally geodesic and therefore act as barrier surfaces. We remove the tubular neighborhoods of $\Gamma$ bounded by these totally geodesic surfaces and denote the result $M \backslash N_{f(t)}(\Gamma)$ by $M^{\prime}$ throughout the remainder of this proof. We assume, after a small isotopy if necessary, that $S$ does not intersect $N_{f(t)}$ for any $t$, and therefore we can (and do) think of $S$ as a surface in $M^{\prime}$. Notice that $M^{\prime}$ is a complete Riemannian manifold with totally geodesic boundary. We will construct the surface $S_{t}$ in $M^{\prime}$, in the same isotopy class as $S$ (also in $M^{\prime}$ ).

If there exists a lower bound on the injectivity radius in $M^{\prime}$ with respect to the $g_{t}$ metric, then the main theorem of [MSY] implies that either such a globally least area surface $S_{t}$ can be found, or $S$ is the boundary of a twisted $I$-bundle over a closed surface in $M^{\prime}$, or else $S$ can be homotoped off every compact set in $M^{\prime}$.

First we show that these last two possibilities cannot occur. If $S$ is nonseparating in $M$, then it intersects some essential loop $\beta$ with algebraic intersection number 1. It follows that $S$ cannot be homotoped off $\beta$ and does not bound an $I$-bundle. Similarly, if $\gamma_{1}, \gamma_{2}$ are distinct geodesics of $\Gamma$ separated from each other by $S$, then the $\gamma_{i}$ 's can be joined by an arc $\alpha$ which has algebraic intersection number 1 with the surface $S$. The same is true of any $S^{\prime}$ homotopic to $S$; it follows that $S$ cannot be homotoped off the arc $\alpha$, nor does it bound an $I$-bundle disjoint from $\Gamma$, and therefore does not bound an $I$-bundle in $M^{\prime}$. 
Now suppose that the injectivity radius on $M^{\prime}$ is not bounded below. We use the following trick. Let $g_{t}^{\prime}$ be obtained from the metric $g_{t}$ by perturbing it on the complement of some enormous compact region $E$ so that it has a flaring end there, and such that there is a barrier $g_{t}^{\prime}$-minimal surface close to $\partial E$, separating the complement of $E$ in $M^{\prime}$ from $S$. Then by MSY there is a globally $g_{t}^{\prime}$ least area surface $S_{t}^{\prime}$, contained in the compact subset of $M^{\prime}$ bounded by this barrier surface. Since $S_{t}^{\prime}$ must either intersect $\beta$ or $\alpha$, by the Bounded Diameter Lemma 1.15, unless the hyperbolic area of $S_{t}^{\prime} \cap E$ is very large, the diameter of $S_{t}^{\prime}$ in $E$ is much smaller than the distance from $\alpha$ or $\beta$ to $\partial E$. Since by hypothesis, $S_{t}^{\prime}$ is the least area for the $g_{t}^{\prime}$ metric, its restriction to $E$ has hyperbolic area less than the hyperbolic area of $S$, and therefore there is an a priori upper bound on its diameter in $E$. By choosing $E$ large enough, we see that $S_{t}^{\prime}$ is contained in the interior of $E$, where $g_{t}$ and $g_{t}^{\prime}$ agree. Thus $S_{t}^{\prime}$ is the globally least area for the $g_{t}$ metric in $M^{\prime}$, and therefore $S_{t}=S_{t}^{\prime}$ exists for any $t$.

The bounded diameter lemma easily implies the following:

Lemma 1.21 (Compact set). There is a fixed compact set $E \subset M$ such that the surfaces $S_{t}$ constructed in Lemma 1.20 are all contained in $E$.

Proof. Since the hyperbolic areas of the $S_{t}$ are all uniformly bounded (by e.g. the hyperbolic area of $S$ ) and are 2-incompressible rel. $\Gamma$, they have uniformly bounded diameter away from $\Gamma$ outside of Margulis tubes. Since for homological reasons they must intersect the compact sets $\alpha$ or $\beta$, they can intersect at most finitely many Margulis tubes. It follows that they are all contained in a fixed bounded neighborhood $E$ of $\alpha$ or $\beta$, containing $\Gamma$.

To extract good limits of sequences of minimal surfaces, one generally needs $a$ priori bounds on the area and the total curvature of the limiting surfaces. Here for a surface $S$, the total curvature of $S$ is just the integral of the absolute value of the (Gauss) curvature over $S$. For minimal surfaces of a fixed topological type in a manifold with sectional curvature bounded above, a curvature bound follows from an area bound by Gauss-Bonnet. However, our surfaces $S_{t}$ are minimal with respect to the $g_{t}$ metrics, which have no uniform upper bound on their sectional curvature, so we must work slightly harder to show that the $S_{t}$ have uniformly bounded total curvature. More precisely, we show that their restrictions to the complement of any fixed tubular neighborhood $N_{\epsilon}(\Gamma)$ have uniformly bounded total curvature.

Lemma 1.22 (Finite total curvature). Let $S_{t}$ be the surfaces constructed in Lemma 1.20. Fix some small, positive $\epsilon$. Then the subsurfaces

$$
S_{t}^{\prime}:=S_{t} \cap M \backslash N_{\epsilon}(\Gamma)
$$

have uniformly bounded total curvature.

Proof. Having chosen $\epsilon$, we choose $t$ large enough so that $r(1-t)<\epsilon / 2$.

Observe firstly that each $S_{t}$ has $g_{t}$ area less than the $g_{t}$ area of $S$, and therefore hyperbolic area less than the hyperbolic area of $S$ for sufficiently large $t$.

Let $\tau_{t, s}=S_{t} \cap \partial N_{s}(\Gamma)$ for small $s$. By the coarea formula (see [Fed, CM, p. 8]) we can estimate

$$
\operatorname{area}\left(S_{t} \cap\left(N_{\epsilon}(\Gamma) \backslash N_{\epsilon / 2}(\Gamma)\right)\right) \geq \int_{\epsilon / 2}^{\epsilon} \operatorname{length}\left(\tau_{t, s}\right) d s .
$$


If the integral of geodesic curvature along a component $\sigma$ of $\tau_{t, \epsilon}$ is large, then the length of the curves obtained by isotoping $\sigma$ into $S_{t} \cap N_{\epsilon}(\Gamma)$ grows very rapidly, by the definition of geodesic curvature.

Since there is an a priori bound on the hyperbolic area of $S_{t}$, it follows that there cannot be any long components of $\tau_{t, s}$ with big integral geodesic curvature. More precisely, consider a long component $\sigma$ of $\tau_{t, s}$. For $l \in[0, \epsilon / 2]$ the boundary $\sigma_{l}$ of the l-neighborhood of $\sigma$ in $S_{t} \cap N_{\epsilon}(\Gamma)$ is contained in $N_{\epsilon}(\Gamma) \backslash N_{\epsilon / 2}(\Gamma)$. If the integral of the geodesic curvature along $\sigma_{l}$ were sufficiently large for every $l$, then the derivative of the length of the $\sigma_{l}$ would be large for every $l$, and therefore the lengths of the $\sigma_{l}$ would be large for all $l \in[\epsilon / 4, \epsilon / 2]$. It follows that the hyperbolic area of the $\epsilon / 2$ collar neighborhood of $\sigma$ in $S_{t}$ would be very large, contrary to existence of an a priori upper bound on the total hyperbolic area of $S_{t}$.

This contradiction implies that for some $l$, the integral of the geodesic curvature along $\sigma_{l}$ can be bounded from above. To summarize, for each constant $C_{1}>0$ there is a constant $C_{2}>0$, such that for each component $\sigma$ of $\tau_{t, \epsilon}$ which has length $\geq C_{1}$ there is a loop

$$
\sigma^{\prime} \subset S_{t} \cap\left(N_{\epsilon}(\Gamma) \backslash N_{\epsilon / 2}(\Gamma)\right)
$$

isotopic to $\sigma$ by a short isotopy, satisfying

$$
\int_{\sigma^{\prime}} \kappa d l \leq C_{2}
$$

On the other hand, since $S_{t}$ is $g_{t}$ minimal, there is a constant $C_{1}>0$ such that each component $\sigma$ of $\tau_{t, \epsilon}$ which has length $\leq C_{1}$ bounds a hyperbolic globally least area disk which is contained in $M \backslash N_{\epsilon / 2}(\Gamma)$. For $t$ sufficiently close to 1 , such a disk is contained in $M \backslash N_{r(1-t)}(\Gamma)$ and therefore must actually be a subdisk of $S_{t}$.

By the coarea formula above, we can choose $\epsilon$ so that $\operatorname{length}\left(\tau_{t, s}\right)$ is a priori bounded. It follows that if $S_{t}^{\prime \prime}$ is the subsurface of $S_{t}$ bounded by the components of $\tau_{t, s}$ of length $>C_{1}$, then we have a priori upper bounds on the area of $S_{t}^{\prime \prime}$, on $\int_{\partial S_{t}^{\prime \prime}} \kappa d l$, and on $-\chi\left(S_{t}^{\prime \prime}\right)$. Moreover, $S_{t}^{\prime \prime}$ is contained in $M \backslash N_{r(1-t)}$, where the metric $g_{t}$ agrees with the hyperbolic metric, so the curvature $K$ of $S_{t}^{\prime \prime}$ is bounded above by -1 pointwise, by Lemma 1.2. By the Gauss-Bonnet formula, this gives an a priori upper bound on the total curvature of $S_{t}^{\prime \prime}$ and therefore on $S_{t}^{\prime} \subset S_{t}^{\prime \prime}$.

Remark 1.23. A more highbrow proof of Lemma 1.22 follows from Theorem 1 of $[\underline{\mathrm{S}}$, using the fact that the surfaces $S_{t}^{\prime}$ are locally least area for the hyperbolic metric, for $t$ sufficiently close to 1 (depending on $\epsilon$ ).

Lemma 1.24 (Limit exists). Let $S_{t}$ be the surfaces constructed in Lemma 1.20. Then there is an increasing sequence

$$
0<t_{1}<t_{2}<\cdots
$$

such that $\lim _{i \rightarrow \infty} t_{i}=1$, and the $S_{t_{i}}$ converge on compact subsets of $M \backslash \Gamma$ in the $C^{\infty}$ topology to some $T^{\prime} \subset M \backslash \Gamma$ with closure $T$ in $M$.

Proof. By definition, the surfaces $S_{t}$ have $g_{t}$ area bounded above by the $g_{t}$ area of $S$. Moreover, since $S$ is disjoint from $\Gamma$, for sufficiently large $t$, the $g_{t}$ area of $S$ is equal to the hyperbolic area of $S$. Since the $g_{t}$ area dominates the hyperbolic area, it follows that the $S_{t}$ have hyperbolic area bounded above, and by Lemma 1.22 , for any $\epsilon$, the restrictions of $S_{t}$ to $M \backslash N_{\epsilon}(\Gamma)$ have uniformly bounded finite total curvature. 
Moreover, by Lemma 1.21 each $S_{t}$ is contained in a fixed compact subset of $M$. By standard compactness theorems (see, e.g., [CiSc]) any infinite sequence $S_{t_{i}}$ contains a subsequence which converges on compact subsets of $E \backslash \Gamma$, away from finitely many points where some subsurface with nontrivial topology might collapse. That is, there might be isolated points $p$ such that for any neighborhood $U$ of $p$, the intersection of $S_{t_{i}}$ with $U$ contains loops which are essential in $S_{t_{i}}$ for all sufficiently large $i$.

But $S$ is 2-incompressible rel. $\Gamma$, so in particular it is incompressible in $M \backslash \Gamma$, and no such collapse can take place. So after passing to a subsequence, a limit $T^{\prime} \subset M \backslash \Gamma$ exists (compare MSY]). Since each $S_{t}$ is a globally least area surface in $M \backslash N_{f(t)}(\Gamma)$ with respect to the $g_{t}$ metric, it is a locally least area surface with respect to the hyperbolic metric on $M \backslash N_{r(1-t)}(\Gamma)$. It follows that $T^{\prime}$ is locally least area in the hyperbolic metric, properly embedded in $M \backslash \Gamma$, and we can define $T$ to be the closure of $T^{\prime}$ in $M$.

Lemma 1.25 (Interpolating isotopy). Let $\left\{t_{i}\right\}$ be the sequence as in Lemma 1.24. Then after possibly passing to a subsequence, there is an isotopy $F: S \times[0,1) \rightarrow$ $M \backslash \Gamma$ such that

$$
F\left(S, t_{i}\right)=S_{t_{i}}
$$

and such that for each $p \in S$ the track of the isotopy $F(p,[0,1))$ either converges to some well-defined limit $F(p, 1) \in M \backslash \Gamma$ or else it is eventually contained in $N_{\epsilon}(\Gamma)$ for any $\epsilon>0$.

Proof. Fix some small $\epsilon$. Outside $N_{\epsilon}(\Gamma)$, the surfaces $S_{t_{i}}$ converge uniformly in the $C^{\infty}$ topology to $T^{\prime}$. It follows that for any $\epsilon$, and for $i$ sufficiently large (depending on $\epsilon$ ), the restrictions of $S_{t_{i}}$ and $S_{t_{i+1}}$ to the complement of $N_{\epsilon}(\Gamma)$ are both sections of the exponentiated unit normal bundle of $T^{\prime} \backslash N_{\epsilon}(\Gamma)$, and therefore we can isotope these subsets of $S_{t_{i}}$ to $S_{t_{i+1}}$ along the fibers of the normal bundle. We wish to patch this partial isotopy together with a partial isotopy supported in a small neighborhood of $N_{\epsilon}(\Gamma)$ to define the correct isotopy from $S_{t_{i}}$ to $S_{t_{i+1}}$.

Let $Z$ be obtained from $N_{\epsilon}(\Gamma)$ by isotoping it slightly into $M \backslash N_{\epsilon}(\Gamma)$ so that it is transverse to $T$, and therefore also to $S_{t_{i}}$ for $i$ sufficiently large. For each $i$, we consider the intersection

$$
\tau_{i}=S_{t_{i}} \cap \partial Z
$$

and observe that the limit satisfies

$$
\lim _{i \rightarrow \infty} \tau_{i}=\tau=T \cap \partial Z
$$

Let $\sigma$ be a component of $\tau$ which is inessential in $\partial Z$. Then for large $i, \sigma$ can be approximated by $\sigma_{i} \subset \tau_{i}$ which are inessential in $\partial Z$. Since the $S_{t_{i}}$ are 2incompressible rel. $\Gamma$, the loops $\sigma_{i}$ must bound subdisks $D_{i}$ of $S_{t_{i}}$. Since $\partial Z$ is a convex surface with respect to the hyperbolic metric, and the $g_{t}$ metric agrees with the hyperbolic metric outside $Z$ for large $t$, it follows that the disks $D_{i}$ are actually contained in $Z \backslash \Gamma$ for large $i$. It follows that $D_{i}$ and $D_{i+1}$ are isotopic by an isotopy supported in $Z \backslash \Gamma$, which restricts to a very small isotopy of $\sigma_{i}$ to $\sigma_{i+1}$ in $\partial Z$.

Let $\sigma$ be a component of $\tau$ which is essential in $\partial Z$. Then so is $\sigma_{i}$ for large $i$. Again, since $S$, and therefore $S_{t_{i}}$ is 2-incompressible rel. $\Gamma$, it follows that $\sigma_{i}$ cannot be a meridian of $\partial Z$ and must actually be a longitude. It follows that there is another essential curve $\sigma_{i}^{\prime}$ in each $\tau_{i}$, such that the essential curves $\sigma_{i}^{\prime}$ and $\sigma_{i}$ 
cobound a subsurface $A_{i}$ in $S_{t_{i}} \cap Z \backslash \Gamma$. After passing to a diagonal subsequence, we can assume that the $\sigma_{i}^{\prime}$ converge to some component $\sigma^{\prime}$ of $\tau$.

By 2-incompressibility, the surfaces $A_{i}$ are annuli. Note that there are two relative isotopy classes of such annuli. By passing to a further diagonal subsequence, we can assume $A_{i}$ and $A_{i+1}$ are isotopic in $Z \backslash \Gamma$ by an isotopy which restricts to a very small isotopy of $\sigma_{i} \cup \sigma_{i}^{\prime}$ to $\sigma_{i+1} \cup \sigma_{i+1}^{\prime}$ in $\partial Z$.

We have shown that for any small $\epsilon$ and any sequence $S_{t_{i}}$, there is an arbitrarily large index $i$ and infinitely many indices $j$ with $i<j$ so that the surfaces $S_{t_{i}}$ and $S_{t_{j}}$ are isotopic, and the isotopy can be chosen to have the following properties:

(1) The isotopy takes $N_{\epsilon}(\Gamma) \cap S_{t_{i}}$ to $N_{\epsilon}(\Gamma) \cap S_{t_{j}}$ by an isotopy supported in $N_{\epsilon}(\Gamma)$

(2) Outside $N_{\epsilon}(\Gamma)$, the tracks of the isotopy are contained in fibers of the exponentiated normal bundle of $T^{\prime} \backslash N_{\epsilon}(\Gamma)$.

Choose a sequence $\epsilon_{i} \rightarrow 0$, and pick a subsequence of the $S_{t_{i}}$ 's and relabel so that $S_{t_{i}}, S_{t_{i+1}}$ satisfy the properties above with respect to $N_{\epsilon_{i}}(\Gamma)$. Then the composition of this infinite sequence of isotopies is $F$.

Remark 1.26. The reason for the circumlocutions in the statement of Lemma 1.25 is that we have not yet proved that $T$ is a limit of the $S_{t}$ as maps from $S$ to $M$. This will follow in $\$ 1.6$, where we analyze the structure of $T$ near a point $p \in \Gamma$ and show it has a well-defined tangent cone.

1.6. Existence of tangent cone. We have constructed $T$ as a subset of $M$ and have observed that away from $\Gamma, T$ is a minimal surface for the hyperbolic metric. We refer to the intersection $T \cap \Gamma$ as the coincidence set. In general, one cannot expect $T$ to be smooth along the coincidence set. However, we show that it does have a well defined tangent cone in the sense of Gromov, and this tangent cone is in fact of a very special form. In particular, this is enough to imply that $T$ exists as the image of a map from $S$ to $M$, and we may extend the isotopy $F: S \times[0,1) \rightarrow M$ to a homotopy $F: S \times[0,1] \rightarrow M$ with $T=F(S, 1)$.

By a tangent cone we mean the following: at each point $p \in T \cap \Gamma$, consider the pair of metric spaces $\left(N_{s}(p), T_{s}(p)\right)$ where $T_{s}(p)$ is the intersection $T_{s}=T \cap N_{s}(p)$. We rescale the metric on this pair by the factor $1 / s$. Then we claim that this sequence of (rescaled) pairs of metric spaces converges in the Gromov-Hausdorff sense to a limit $(B, C)$ where $B$ is the unit ball in Euclidean 3 -space, and $C$ is the cone (to the origin) over a great bigon in the unit sphere. Here by a great bigon we mean the union of two spherical geodesics joining antipodal points in the sphere. In fact we do not quite show that $T$ has this structure, but rather that each local branch of $T$ has this structure. Here we are thinking of the $\operatorname{map} F(\cdot, 1): S \rightarrow M$ whose image is $T$, and by "local branch" we mean the image of a regular neigborhood of a point preimage.

Lemma 1.27 (Tangent cone). Let $T$ be as constructed in Lemma 1.24. Let $p \in$ $T \cap \Gamma$. Then near $p, T$ is a (topologically immersed) surface, each local branch of which has a well-defined tangent cone, which is the cone on a great bigon.

Proof. We use what is essentially a curve-shortening argument. For each small $s$, define

$$
T_{s}=\partial N_{s}(p) \cap T
$$


For each point $q \in T \backslash \Gamma$, we define $\alpha(q)$ to be the angle between the tangent space to $T$ at $q$ and the radial geodesic through $q$ emanating from $p$. By the coarea formula, we can calculate

$$
\operatorname{area}\left(T \cap N_{s}(p)\right)=\int_{0}^{s} \int_{T_{t}} \frac{1}{\cos (\alpha)} d l d t \geq \int_{0}^{s} \operatorname{length}\left(T_{t}\right) d t,
$$

where $d l$ denotes the length element in each $T_{t}$. Note that this estimate implies that $T_{t}$ is rectifiable for a.e. $t$. We choose $s$ to be such a rectifiable value.

Now, each component $\tau$ of $T_{s}$ is a limit of components $\tau_{i}$ of $S_{t_{i}} \cap \partial N_{s}(p)$ for large $i$. By 2-incompressibility of the $S_{t_{i}}$, each $\tau_{i}$ is a loop bounding a subdisk $D_{i}$ of $S_{t_{i}}$ for large $i$.

Now, $\partial N_{s}(p)$ is convex in the hyperbolic metric, though not necessarily in the $g_{t}$ metric. By cutting out the disks $\partial N_{s}(p) \cap N_{r(1-t)}(\Gamma)$ and replacing them with the disks $D^{ \pm}$orthogonal to $\Gamma$ which are totally geodesic in both the $g_{t}$ and the hyperbolic metrics, we can approximate $\partial N_{s}(p)$ by a surface $\partial B$ bounding a ball $B \subset N_{s}(p)$ which is convex in the $g_{s}$ metric for all $s \geq t$. The ball $B$ is illustrated in Figure 1.
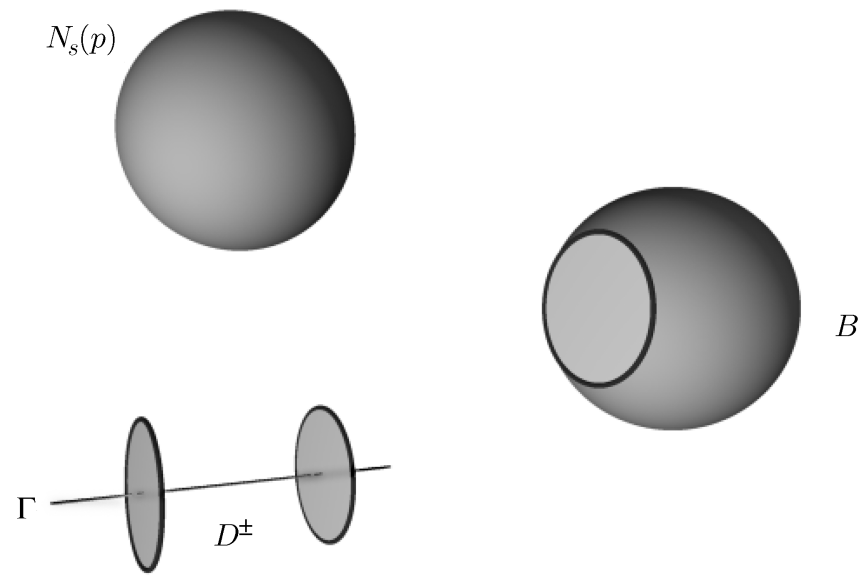

Figure 1. The ball $B$ has boundary which is convex in both the hyperbolic and the $g_{s}$ metrics for all $s \geq t$.

Note that after lifting $B$ to the universal cover, there is a retraction onto $B$ which is length nonincreasing, in both the $g_{t}$ and the hyperbolic metric. This retraction projects along the fibers of the product structure on $N_{r(1-t)}(\Gamma)$ to $D^{ \pm}$, and outside $N_{r(1-t)}(\Gamma)$, it is the nearest point projection to $\partial B \backslash D^{ \pm}$.

Let $\tau_{i}^{\prime}$ be the component of $S_{t_{i}} \cap \partial B$ approximating $\tau_{i}$, and let $D_{i}^{\prime}$ be the subdisk of $S_{t_{i}}$ which it bounds.

Then the disk $D_{i}^{\prime}$ must be contained in $B$, or else we could decrease its $g_{t}$ and hyperbolic area by the retraction described above. The disks $D_{i}^{\prime}$ converge to the component $D \subset T^{\prime}$ bounded by $\tau$, and the hyperbolic areas of the $D_{i}^{\prime}$ converge to the hyperbolic area of $D$.

Note that $B$ as above is really shorthand for $B_{t}$, since it depends on a choice of $t$. Similarly we have $\tau_{t}$ and $D_{t}$. Since the component $D_{t} \subset T^{\prime}$ bounded by $\tau_{t}$ is 
contained in $B_{t}$ for all $t$, the component $D \subset T^{\prime}$ bounded by $\tau \subset \partial N_{s}$ is contained in $N_{s}$, since $B_{t} \rightarrow N_{s}$ as $t \rightarrow 1$. So we can, and do, work with $N_{s}(p)$ instead of $B$ in the sequel.

Now, let $D^{\prime}$ be the cone on $\tau$ to the point $p$. $D^{\prime}$ can be perturbed an arbitrarily small amount to an embedded disk $D^{\prime \prime}$, and therefore by comparing $D^{\prime \prime}$ with the $D_{i}^{\prime}$, we see that the hyperbolic area of $D^{\prime}$ must be at least as large as that of $D$. Note that this perturbation can be taken to move $D^{\prime}$ off $\Gamma$ and can be approximated by perturbations which miss $\Gamma$. Similar facts are true for all the perturbations we consider in the sequel.

Since this is true for each component $\tau$ of $T_{s}$, by abuse of notation we can replace $T$ by the component of $T \cap N_{s}(p)$ bounded by a single mapped in circle $\tau$. This will be the local "branch" of the topologically immersed surface $T$. We use this notational convention for the remainder of the proof of the lemma. Note that the inequality above still holds. It follows that we must have

$$
\operatorname{area}\left(T \cap N_{s}(p)\right) \leq \int_{0}^{s} \operatorname{length}\left(T_{s}\right) \frac{\sinh (t)}{\sinh (s)} d t=\operatorname{area}\left(\text { cone on } T_{s}\right) .
$$

Now, for each sphere $\partial N_{s}(p)$, we let $\phi$ be the projection, along hyperbolic geodesics, to the unit sphere $S^{2}$ in the tangent space at $p$. For each $t \in(0,1]$, define

$$
\left\|T_{t}\right\|=\operatorname{length}\left(\phi\left(T_{t}\right)\right)=\frac{\operatorname{length}\left(T_{t}\right)}{\sinh (t)} .
$$

It follows from the inequalities above that for some intermediate $s^{\prime}$ we must have

$$
\left\|T_{s^{\prime}}\right\| \leq\left\|T_{s}\right\|
$$

with equality iff $T \cap N_{s}(p)$ is equal to the cone on $T_{s}$.

Now, the cone on $T_{s}$ is not locally least area for the hyperbolic metric in $N_{s}(p) \backslash \Gamma$ unless $T_{s}$ is a great circle or geodesic bigon in $\partial N_{s}(p)$ (with endpoints on $\left.\partial N_{s}(p) \cap \Gamma\right)$, in which case the lemma is proved. To see this, just observe that a cone has vanishing principal curvature in the radial direction, so its mean curvature vanishes iff it is totally geodesic away from $\Gamma$.

So we may suppose that for any $s$ there is some $s^{\prime}<s$ such that $\left\|T_{s^{\prime}}\right\|<\left\|T_{s}\right\|$. Therefore we choose a sequence of values $s_{i}$ with $s_{i} \rightarrow 0$ such that $\left\|T_{s_{i}}\right\|>\left\|T_{s_{i+1}}\right\|$, such that $\left\|T_{s_{i}}\right\|$ converges to the infimal value of $\left\|T_{t}\right\|$ with $t \in(0, s]$, and such that $\left\|T_{s_{i}}\right\|$ is the minimal value of $\left\|T_{t}\right\|$ on the interval $t \in\left[s_{i}, 1\right]$. Note that for any small $t$, the cone on $T_{t}$ has area

$$
\operatorname{area}\left(\text { cone on } T_{t}\right)=\frac{t}{2} \text { length }\left(T_{t}\right)+O\left(t^{3}\right)=\frac{t^{2}}{2}\left\|T_{t}\right\|+O\left(t^{3}\right) .
$$

The set of loops in the sphere with length bounded above by some constant, parameterized by arclength, is compact, by the Arzela-Ascoli theorem, and so we can suppose that the $\phi\left(T_{s_{i}}\right)$ converge in the Hausdorff sense to a loop $C \subset S^{2}$.

Claim. $C$ is a geodesic bigon.

Proof. We suppose not and will obtain a contradiction.

We fix notation: for each $i$, let $C_{i}$ denote the inverse image $\phi^{-1}(C)$ under $\phi$ : $\partial N_{s_{i}}(p) \rightarrow S^{2}$. So $C_{i}$ is a curve in $\partial N_{s_{i}}(p)$. By the cone on $C_{i}$ we mean the union of the hyperbolic geodesic segments in $N_{s_{i}}(p)$ from $C_{i}$ to $p$. By the cone on $C$ we 
mean the union of the geodesic segments in the unit ball in Euclidean 3-space from $C \subset S^{2}$ to the origin. For each $i$, we have an estimate

$$
\text { area }\left(\text { cone on } C_{i}\right)=s_{i}^{2} \text { area }(\text { cone on } C)+O\left(s_{i}^{3}\right) \text {. }
$$

For each $i$, let $T^{i}$ denote the surface obtained from $T \cap N_{s_{i}}(p)$ by rescaling metrically by $1 / s_{i}$. Then $T^{i}$ is a surface with boundary contained in a ball of radius 1 in a space of constant curvature $-s_{i}^{2}$. Moreover, it enjoys the same least area properties as $T \cap N_{s_{i}}(p)$.

By the monotonicity property of the $\left\|T_{s_{i}}\right\|$ and the coarea formula, we have an inequality

$$
\lim _{i \rightarrow \infty} \operatorname{area}\left(T^{i}\right) \geq \operatorname{area}(\text { cone on } C) .
$$

On the other hand, since each $T^{i}$ is least area, we have an estimate

$$
\frac{1}{2}\left\|T_{s_{i}}\right\|+O\left(s_{i}\right)=\frac{\operatorname{area}\left(\text { cone on } T_{s_{i}}\right)}{s_{i}^{2}} \geq \operatorname{area}\left(T^{i}\right) .
$$

It follows that the limit of the area of the $T^{i}$ is actually equal to the area of the cone on $C$.

On the other hand, since the $\phi\left(T_{s_{i}}\right)$ converge to $C$, for sufficiently large $i$ we can find an immersed annulus $A_{i}$ in $S^{2}$ with area $\leq \kappa$ for any positive $\kappa$, which is the track of a homotopy (in $\left.S^{2}\right)$ from $\phi\left(T_{S_{i}}\right)$ to $C$. We let $\phi^{-1}\left(A_{i}\right)$ denote the corresponding annulus in $\partial N_{s_{i}}(p)$.

We can build a new immersed surface bounded by $T_{s_{i}}$ which is the union of this annulus $\phi^{-1}\left(A_{i}\right)$ with the cone on $C_{i}$. This surface can be perturbed an arbitrarily small amount, away from $\Gamma$, to an embedded surface $F^{i}$. After rescaling $F^{i}$ by $1 / s_{i}$, we get a surface $G^{i}$ with the same boundary as $T^{i}$ of area equal to

$$
\operatorname{area}\left(G^{i}\right)=\operatorname{area}(\text { cone on } C)+\kappa+O\left(s_{i}\right) \text {. }
$$

Since $T^{i}$ is locally least area, it follows that for any $\kappa>0$, for sufficiently large $i$ (depending on $\kappa$ ),

$$
\text { area }(\text { cone on } C)+2 \kappa \geq \operatorname{area}\left(G^{i}\right) \geq \operatorname{area}\left(T^{i}\right) \geq \operatorname{area}(\text { cone on } C)-\kappa \text {. }
$$

The surface $F^{i}$ contains a subsurface which is the cone on $C_{i}$. Since by hypothesis, $C$ is not a geodesic bigon, the cone on $C$ can be perturbed by a compactly supported perturbation to a surface whose area is smaller than that of the cone on $C$ by some definite amount $\epsilon$. Similarly, the cone on $C_{i}$ can be perturbed by a compactly supported perturbation to a surface whose area is smaller than the cone on $C_{i}$ by $\epsilon\left(s_{i}\right)^{2}$ where $\epsilon$ is independent of $i$. After rescaling by $1 / s_{i}$, it follows that $G^{i}$ can be perturbed by a compactly supported perturbation to $H^{i}$ with the same boundary as $G^{i}$ and $T^{i}$, for which

$$
\operatorname{area}\left(H^{i}\right) \leq \operatorname{area}\left(G^{i}\right)-\epsilon
$$

where $\epsilon$ is independent of $i$. Since $\kappa$ may be chosen as small as we like, we choose $3 \kappa<\epsilon$. Then for sufficiently large $i$ we get

$$
\operatorname{area}\left(H^{i}\right)<\operatorname{area}\left(T^{i}\right),
$$

which contradicts the least area property of $T^{i}$. This contradiction shows that $C$ is actually a geodesic bigon and completes the proof of the claim. 
We now complete the proof of Lemma 1.27

Let $C \subset S^{2}$ be this geodesic bigon. Then inside an $\epsilon$-neighborhood of $C$ in $S^{2}$, we can find a pair of curves $C^{ \pm}$, where $C^{+}$is convex, and $C^{-}$is convex except for two acute angles on $T \Gamma \cap S^{2}$. For each $i$, let $C_{i}^{ \pm} \subset \partial N_{s_{i}}(p)$ be the inverse image of $C^{ \pm}$under $\phi$.

The cone on $C_{i}^{ \pm}$is a pair of barrier surfaces in $N_{s}(p)$. In particular, once $\phi\left(T_{s_{i}}\right)$ and $\phi\left(T_{s_{i+1}}\right)$ are both trapped between $C^{+}$and $C^{-}$, the same is true of $\phi\left(T_{s^{\prime}}\right)$ for all $s^{\prime} \in\left[s_{i+1}, s_{i}\right]$. This is enough to establish the existence of the tangent cone.

Notice that Lemma 1.27 actually implies that $T$ is a rectifiable surface in $M$, which is a local (topological) embedding. In particular, this shows that the isotopy $F: S \times[0,1) \rightarrow M$ constructed in Lemma1.25 can be chosen to limit to a homotopy $F: S \times[0,1] \rightarrow M$ such that $F(S, 1)=T$.

1.7. The thin obstacle problem. From the proof of Lemma 1.27, we see that $T$ exists as a $C^{0}$ map, which by abuse of notation we denote $u: T \rightarrow M$. One may immediately improve the regularity of $u$. From the construction of $T$, it is standard to show that $u$ is actually in the Sobolev space $H^{1,2}$ — that is, the derivative $d u$ is defined, and is $L^{2}$, in the sense of distribution; see $\mathrm{Mor}$ for a rigorous definition of Sobolev spaces and basic properties.

To see this, observe that $u$ is a limit of maps $F\left(\cdot, t_{i}\right): S \rightarrow M$ which are minimal for the $g_{t}$ metric and therefore are $L^{2}$ energy minimizers for the conformal structure on $S$ pulled back by $F\left(\cdot, t_{i}\right)$. If the set of conformal structures obtained in this way is precompact, one may extract a limit and therefore bound the $L^{2}$ norm of $d u$ in terms of the $L^{2}$ norms of the derivatives of any $F\left(\cdot, t_{i}\right)$. How can the sequence of conformal structures fail to be precompact? This happens if and only if the conformal structures degenerate by a neck pinch. But the 2-incompressibility of $S$ rel. $\Gamma$ implies that there is a lower bound on the length of the image of any essential curve in $S$. It follows that the $L^{2}$ norm of the derivative blows up along such a pinching neck, contrary to the energy minimizing property. So no such degeneration can occur, and $u$ is in $H^{1,2}$ as claimed. This argument is essentially contained in [SY] (see e.g. Lemma 3.1, p. 134), and one may consult this paper for details.

We need to establish further regularity of $d u$ along $\Gamma$ in the following sense. Recall that we are calling $L:=T \cap \Gamma$ the coincidence set. For each local sheet of $T$, we want $u$ to be $C^{1}$ along the interior of $L$ from either side, and at a noninterior point of $L$, we want $u$ to be $C^{1}$ on the nose.

Now, if $I \subset L$ is an interval, then the reflection principle (see Oss) implies that each local sheet $T^{+}$of $T$ with $\partial T^{+}=I$ can be analytically continued to a minimal surface across $I$, by taking another copy of $T^{+}$, rotating it through angle $\pi$ along the axis $I$ and gluing it to the original $T^{+}$along $I$. It follows that $d u$ is real analytic from either side along the interior of $L$. Note that if the tangent cone at a point $p$ is not literally a tangent plane, then an easy comparison argument implies that $p$ is an interior point of the coincidence set. See [N, p. 90] for a fuller discussion.

Noninterior points of $L$ are more difficult to deal with, and we actually want to conclude that $d u$ is continuous at such points. Fortunately, this is a well-known problem in the theory of variational problems, known as the Signorini problem, or the (two-dimensional) thin obstacle problem. 
In the literature, this problem is usually formulated in the following terms:

Thin Obstacle Problem. Let $\Omega$ be a bounded open subset of $\mathbb{R}^{2}$, and $A$ an oriented line contained in $\Omega$. Let $\psi: A \rightarrow \mathbb{R}$ and $g: \partial \Omega \rightarrow \mathbb{R}$ be given, with $g \geq \psi$ on $\partial \Omega \cap A$. Define

$$
\mathbb{K}=\left\{v \in g+H_{0}^{1, p} \mid v \geq \psi \text { on } A\right\}
$$

Minimize

$$
J(u)=\int F(x, u, \nabla u) d x
$$

over $u \in \mathbb{K}$.

Here $H^{1, p}$ denotes the usual Sobolev space over $\Omega$ for the $L^{p}$ norm, with zero boundary conditions.

The main conditions typically imposed on $F$ are sufficient regularity of $F$ and its partial derivatives (Lipschitz is usually enough) and ellipticity, meaning that the matrix $\left(F_{i k}\right)_{i, k=1,2}$ of the second partial derivatives of $F(x, u, \eta)$ with respect to $\eta \in \mathbb{R}^{2}$ is uniformly positive definite on compact subsets of $\bar{\Omega} \times \mathbb{R}^{2+1}$ (see [Fre, p. 281] for details). Roughly speaking, $F$ is elliptic if the critical functions of the functional $J$ satisfy a "mean value property"; i.e., the value at each point is a weighted average of the value at nearby points.

For example, if we want the graph of $u$ to be a (Euclidean) minimal surface away from $\psi(A)$, then the formula for $F$ is $F=\left(1+|\nabla u|^{2}\right)^{1 / 2}$, which is real analytic and elliptic. The definition of $F$ for a nonparametric minimal surface in exponential coordinates on hyperbolic space is more complicated, but certainly $F$ is real analytic and elliptic in the sense of Frehse.

See Figure 2 for an example of the graph of a function solving the Dirichlet thin obstacle problem, where $\left.\psi\right|_{A}$ is constant. This surface is visually indistinguishable from the graph of the function solving the unparameterized minimal surface thin obstacle problem with the same boundary and obstacle data, but for computer implementation, the Dirichlet problem is less computationally costly.

The next theorem establishes not only the desired continuity of $\partial u$, but actually gives an estimate for the modulus of continuity. The following is a restatement of Theorem 1.3 on page 26 of $\mathrm{Ri}$ in our context:

Theorem 1.28 (Richardson [Ri] regularity of thin obstacle). Let $u$ be a solution to the thin obstacle problem for $F$ elliptic in the sense of Frehse and $p \in[1, \infty]$, and suppose that $\partial \Omega, \psi, g$ are smooth. Then $\partial u$ is continuous along $A$ in the tangent direction, one-sided continuous in the normal direction on either side, and continuous in the normal direction at a noninterior point. Furthermore, $\partial u$ is Hölder continuous, with exponent $1 / 2$; i.e., the modulus of continuity of $\partial u$ is $O\left(t^{1 / 2}\right)$.

Remark 1.29. Note that $C^{1+1 / 2}$ is actually best possible. Consider the function $u: z \rightarrow \operatorname{Im}\left(z^{3 / 2}\right)$ for $u \in \mathbb{C}$ slit along the positive real axis, where we take the branch which is negative sufficiently close to the slit. This solves a thin obstacle problem for the Dirichlet integral and is only $C^{1+1 / 2}$ at $z=0$.

Remark 1.30. For our applications, the fact that $u$ is $C^{1+1 / 2}$ is more than necessary. In fact, all we use is that $u$ is $C^{1}$. This is proved (with a logarithmic modulus of continuity for $d u$ ) by Fre, and (with a Hölder modulus of continuity for $d u$ ) in arbitrary dimension by $[\mathrm{K}$. 


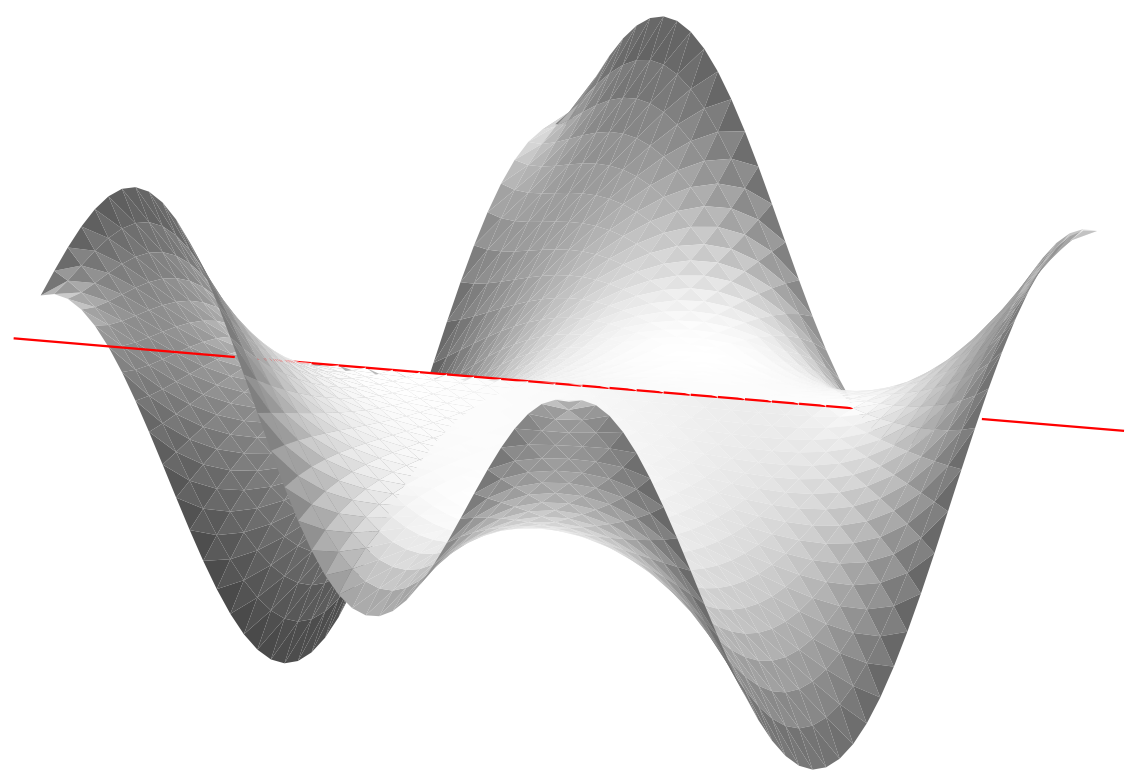

FIgURE 2. The graph of a function solving the thin obstacle problem

We apply this theorem to our context:

Lemma 1.31 (Regularity along coincidence set). For $u: T \rightarrow M$ defined as above, the derivative $d u$ along local sheets of $T$ is continuous from each side along the coincidence set $L$, and continuous at noninterior points.

Proof. If $p$ is an interior point of $L$, this follows by the reflection principle. Otherwise, by Lemma 1.27 and the discussion above, the tangent cone is a plane $\pi$ in the tangent space at $p$.

We show how to choose local coordinates in a ball $B$ near each point $p \in L$ such that $B \cap \Gamma$ is the $x$-axis, each local sheet of $T$ is the graph of a function $u: \Omega \rightarrow \mathbb{R}$, and $u$ is nonnegative along the $x$-axis. Let $\gamma=B \cap \Gamma$, and let $\gamma^{\prime}$ be another geodesic through $p$ orthogonal to $\gamma$ and tangent to $\pi$. Let $\mathcal{F}$ and $\mathcal{G}$ be foliations of $B$ by totally geodesic planes orthogonal to $\gamma$ and $\gamma^{\prime}$ respectively. Then each leaf of $\mathcal{F}$ is totally geodesic for both the hyperbolic and the $g_{t}$ metric for all $t$, and each leaf of $\mathcal{G}$ is totally geodesic for the $g_{t}$ metric for sufficiently large $t$. It follows that $T$ has no source or sink singularities with respect to either foliation. Since $T \cap B$ is a (topological) disk, by reasons of Euler characteristic it can have no saddle singularities either, and therefore no singularities at all. We let $\mathcal{F}$ and $\mathcal{G}$ be level sets of two coordinate functions on $B$. Define a third coordinate function to be (signed) hyperbolic distance to the plane containing $\gamma$ and $\gamma^{\prime}$ and observe that $u$ is a graph in these coordinates.

It follows that $u$ solves an instance of the thin obstacle problem, and by Theorem 1.28 or by [Fre or $[\mathrm{K}]$ the desired regularity of $d u$ follows. 
Remark 1.32. The structure of the coincidence set is important to understand, and it has been studied by various authors. Hans Lewy [Lew showed that for $J$ the Dirichlet integral and $\psi$ analytic, the coincidence set is a finite union of points and intervals. Athanasopoulos Ath proved the same result for the minimal surface question, for symmetric domain $\Omega$ and obstacle $A$, but his (very short and elegant) proof relies fundamentally on the symmetry of the problem, and we do not see how it applies in our context.

Note that if the Hausdorff dimension of the coincidence set is strictly $<1$, then since $T$ is $C^{1+1 / 2}$ (and therefore Lipschitz) along this coincidence set, the theory of removable singularities implies that $T$ is actually real analytic along $\Gamma$. It follows in this case that the coincidence set consists of a finite union of isolated points, and that $T$ is actually a minimal surface. See, e.g., $\mathrm{Car}$ for details.

Remark 1.33. Existence results for the thin obstacle problem for minimal surfaces with analytic obstacles (see, e.g., $[\mathrm{Ri},[\mathrm{K}],[\mathrm{N}]$ ) give an alternative proof of the existence of the limit $T$. Given $S$, we can shrinkwrap $S$ near $\Gamma$ in small balls by using existence for the thin obstacle problem, and away from $\Gamma$ by replacing small disks with least area embedded disks with the same boundary. The argument of [HS] implies that for $S$ 2-incompressible, this converges to a surface $T$.

1.8. CAT $(-1)$ property. We have shown that $T$ satisfies all the properties of the conclusion of Theorem 1.10, except that we have not yet shown that it is intrinsically $\operatorname{CAT}(-1)$. In this subsection we show that after possibly replacing $T$ by a new surface with the same properties, we can insist that $T$ is $\operatorname{CAT}(-1)$ with respect to the path metric induced from $M$.

Lemma 1.34 (CAT $(-1)$ property). After possibly replacing $T$ by a new immersed surface with the same properties, $T$ is $C A T(-1)$ with respect to the path metric induced from $M$.

Proof. To show that $T$ is $\mathrm{CAT}(-1)$ we will show that there is no distributional positive curvature concentrated along the coincidence set $L$. Since $T \backslash \Gamma$ is a minimal surface, the curvature of $T$ is bounded above by -1 on this subset. It will follow by Gauss-Bonnet that $T$ is $\operatorname{CAT}(-1)$.

We first treat a simpler problem in Euclidean 3-space, which we denote by $\mathbb{R}^{3}$. Let $\Sigma$ be an embedded surface in $\mathbb{R}^{3}$ which is $C^{3}$ outside a subset $X$ which is contained in a geodesic $\gamma$ in $\mathbb{R}^{3}$, and which is $C^{1}$ along $X$ from either side along the interior of $X$, and $C^{1}$ at noninterior points of $X$. Then we claim, for each subsurface $R \subset \Sigma$ with $C^{3}$ boundary $\partial R \subset \Sigma \backslash X$, that

$$
\int_{R \backslash X} K_{\Sigma}=2 \pi \chi(R)-\int_{\partial R} \kappa d l
$$

Compare Lemma 1.3

In other words, we want to show that $X$ is a "removable singularity" for $R$, at least with respect to the Gauss-Bonnet formula.

Let $\phi: R \backslash X \rightarrow S^{2}$ denote the Gauss map, which takes each point $p \in R$ to its unit normal, in the unit sphere of $S^{2}$. Then $K_{\Sigma}$ is the pullback of the area form by $\phi$. Let $\bar{R}$ denote the completion of $R \backslash X$ with respect to the path metric. Then $\bar{R}$ is obtained from $R$ by cutting it open along each interval in $R \cap X$ and sewing in two copies of the interval thereby removed. Notice that there is a natural forgetful $\operatorname{map} \bar{R} \rightarrow R$. 
By the assumptions about the regularity of $R$, the Gauss map $\phi$ actually extends to a continuous map $\phi: \bar{R} \rightarrow S^{2}$. Moreover, since $X$ is contained in a geodesic $\gamma$ of $\mathbb{R}^{3}$, the image $\phi(\bar{R} \backslash R)$ is contained in a great circle $C$ in $S^{2}$.

For each boundary component $\tau$ of $\bar{R}$, we claim that the map $\phi: \tau \rightarrow C$ has degree zero. For, otherwise, by a degree argument, there are points $p^{ \pm} \in \bar{R}$ which map to the same point in $p$, for which $\phi\left(p^{+}\right)=-\phi\left(p^{-}\right)$, and the graphs of $\phi\left(p^{+}\right)$ and $-\phi\left(p^{-}\right)$locally have a nonzero algebraic intersection number. It follows that the local sheets of $R$ from either side must actually intersect along $p$, contrary to the fact that $\Sigma$ is embedded. It follows that we can sew in a disk to $\bar{R}$ along each boundary component to get a surface $\bar{R}^{\prime}$ homeomorphic to $R$, with $\partial \bar{R}^{\prime}=\partial R$, and extend $\phi$ to $\phi: \bar{R}^{\prime} \rightarrow S^{2}$ by mapping each such disk into $C$.

Now, the surface $\bar{R}^{\prime}$ can be perturbed slightly in a neighborhood of $X$ to a new surface $\bar{R}^{\prime \prime}$ which is $C^{3}$ in $\mathbb{R}^{3}$, in such a way that the Gauss map of $\bar{R}^{\prime \prime}$ is a perturbation of $\phi$. So the usual Gauss-Bonnet formula (Lemma 1.3) shows that

$$
\int_{\bar{R}^{\prime \prime}} K=2 \pi \chi(R)-\int_{\partial R} \kappa d l \text {. }
$$

But $\int_{\bar{R}^{\prime \prime}} K$ is just the integral of the area form on $S^{2}$ pulled back by the Gauss map; it follows that

$$
\int_{\bar{R}^{\prime \prime}} K=\int_{S^{2}} \operatorname{degree}\left(\phi\left(\bar{R}^{\prime}\right)\right)
$$

and

$$
\int_{S^{2}} \operatorname{degree}\left(\phi\left(\bar{R}^{\prime \prime}\right)\right)=\int_{S^{2}} \operatorname{degree}\left(\phi\left(\bar{R}^{\prime}\right)\right)
$$

since one map is obtained from the other by a small perturbation supported away from the boundary. Since the measure of $C$ is zero, this last integral is just equal to

$$
\int_{S^{2} \backslash C} \operatorname{degree}\left(\phi\left(\bar{R}^{\prime}\right)\right)=\int_{R \backslash X} K
$$

and the claim is proved.

Now we show how to apply this to our shrinkwrapped surface $T$. We use the following trick. Let $j_{t}$ with $t \in[0,1)$ be a family of metrics on $M$, conformally equivalent to the hyperbolic metric, which agree with the hyperbolic metric outside $N_{r(1-t)}$, which are Euclidean on $N_{r(1-t) / 2}$, and which have curvature pinched between -1 and 0 , and are rotationally and translationally symmetric along the core geodesic. Then we let $T_{t}$ be the surface obtained by shrinkwrapping $S$ with respect to the $j_{t}$ metric. That is, we let $g_{s, t}$ be a family of metrics as in Definition 1.17 which agree with the $j_{t}$ metric outside $N_{r(1-t)(1-s)}$, construct minimal surfaces $S_{s, t}$ as in Lemma 1.20 and so on, limiting to the immersed surface $T_{t}$ which is minimal for the $j_{t}$ metric on $M \backslash \Gamma$, and $C^{1+1 / 2}$ along $T_{t} \cap \Gamma$. Arguing locally as above, we see that small subsurfaces of $T_{t}$ contained in the Euclidean tubes $N_{r(1-t) / 2}$ satisfy Gauss-Bonnet in the complement of the coincidence set. By Lemma 1.2, the surfaces $T_{t}$ all have curvature bounded above by 0 , and bounded above by -1 outside $N_{r(1-t)}$. By Gauss-Bonnet for geodesic triangles, $T_{t}$ is CAT(0), and actually CAT(-1) outside $N_{r(1-t)}$.

Now take the limit as $t \rightarrow 1$. Some subsequence of the surfaces $T_{t}$ converges to a limit which by abuse of notation we denote $T$. Note that this is not necessarily the same as the surface $T$ constructed in previous sections, but it enjoys the same 
properties. Again, by Gauss-Bonnet for geodesic triangles, the limit is actually $\mathrm{CAT}(-1)$, and the lemma is proved.

This completes the proof of Theorem 1.10 .

Problem 1.35. Develop a simplicial or PL theory of shrinkwrapping.

Remark 1.36. Since this paper appeared in preprint form, Soma has developed some elements of a PL theory of shrinkwrapping; see [Som. This theory proves a PL analogue of Theorem 1.10 .

\section{The MAin CONSTRUCTION LEMMA}

The purpose of this section is to state the main construction Lemma 2.3 and show how it follows easily from Theorem 1.10.

2.1. Shrinkwrapping in covers. Let $N$ be a complete, orientable, parabolic free hyperbolic 3-manifold, and let $\Gamma$ be a finite collection of pairwise disjoint simple closed geodesics in $N$, just as in the statement of Theorem 1.10. For the purposes of introducing the Main Construction Lemma, we will assume that $N$ has a single end $\mathcal{E}$. We consider the family of $g_{t}$ and $g_{s, t}$ metrics, as in Definition 1.17 and Lemma 1.34

Suppose there is an embedded surface $\partial W$ in $N \backslash \Gamma$ which separates off the end of $N$ from a compact submanifold $W \subset N$. Let $X$ be a covering space of $W$ (possibly infinite). The preimage of the geodesics $\Gamma$ are a collection of locally finite geodesics $\hat{\Gamma} \subset X$, some of which might be finite, and some infinite. Let $\Delta \subset \hat{\Gamma}$ be some nonempty collection, consisting entirely of simple closed geodesics. Then we can consider a second surface $S \subset X \backslash \Delta$ and can ask whether it is possible to shrinkwrap $S$ rel. $\Delta$. Notice that we cannot directly apply Theorem 1.10 because the hyperbolic manifold $X$ is not complete, and therefore a shrinkwrap representative of $S$ might not exist. However, we note that for each metric $g_{t}$ on $N$, we get a $g_{t}$ locally least area representative $\partial W_{t}$ isotopic to $\partial W$. The submanifold $W_{t}$ of $N$ bounded by $\partial W_{t}$ lifts to a covering space $X_{t}$ which is homeomorphic to $X$. The metric $g_{t}$ pulls back to a metric on $X_{t}$, which by abuse of notation we also refer to as $g_{t}$. Then $\partial X_{t}$, which is a lift of $\partial W_{t}$, is $g_{t}$ locally least area and therefore acts as a barrier surface. It follows that we can find, for each $t$, a surface $S_{t}$ in the isotopy class of $S$ in $X_{t} \backslash N_{f(t)}(\Delta)$ which is globally $g_{t}$ least area among all such surfaces (compare with the statement of Lemma 1.20).

The theory of shrinkwrapping developed in $\S 1$ goes through almost identically for the surfaces $S_{t}$ with one important exception: the metric $g_{t}$ on $X_{t}$ does not agree with the hyperbolic metric away from $\Delta$ and $\partial X_{t}$, but rather is deformed along the other geodesics $\hat{\Gamma} \backslash \Delta$. It follows that we should take care to analyze the quality of the surfaces $S_{t}$ and their limit $S^{\prime}$ near components of $\hat{\Gamma} \backslash \Delta$.

Fortunately the situation is as simple as it could be:

Lemma 2.1 (Superfluous geodesics invisible). With notation and definitions as above, in a neighborhood of a point $p$ on $\hat{\Gamma} \backslash \Delta$, the surface $S^{\prime}$ is a locally least area surface for the hyperbolic metric.

Proof. Suppose not. Then there is a compactly supported perturbation $F$ of $S^{\prime}$ which agrees with $S^{\prime}$ outside a fixed neighborhood of $p$, and which has strictly less 
hyperbolic area than $S^{\prime}$, so that

$$
\operatorname{area}\left(S^{\prime}\right)-\operatorname{area}(F) \geq \epsilon
$$

for some positive constant $\epsilon$. After another small perturbation of $F$ to $F^{\prime}$, which can be taken to increase the hyperbolic area as little as required, we can assume that $F^{\prime}$ is transverse to $\Gamma$ near $p$, intersecting it in $n$ points for some finite $n$ and satisfying

$$
\operatorname{area}\left(S^{\prime}\right)-\operatorname{area}\left(F^{\prime}\right) \geq \epsilon / 2 \text {. }
$$

By property (3) of the $g_{t}$ metric (see Lemma 1.18) the $g_{t}$ area of $F^{\prime}$ is at most equal to the hyperbolic area plus $n C(1-t)^{2}$, for some constant $C$ independent of $t$. For sufficiently small $t$,

$$
n C(1-t)^{2}<\epsilon / 2
$$

and therefore the $g_{t}$ area of $F^{\prime}$ is less than the hyperbolic area of $S^{\prime}$, which is less than the $g_{t}$ area of $S^{\prime}$, thereby contradicting the global $g_{t}$ minimality of $S^{\prime}$ in its isotopy class in $X_{t} \backslash N_{f(t)}(\Delta)$.

This contradiction proves the lemma.

A similar argument holds for the $g_{t, s}$ metric in place of the $g_{t}$ metric, and therefore by means of Lemma 2.1 we can shrinkwrap in covers, obtaining $\mathrm{CAT}(-1)$ surfaces in the limit.

Remark 2.2. One should think of Lemma 2.1 as a kind of "removable singularity" theorem for transverse obstacles. Compare with the following physical experiment: one knows from experience that very thin needles can be pushed through soap bubbles without popping them or distorting their geometry. (Try it!)

2.2. The main construction lemma. We now state and prove the main construction lemma. The context of this lemma is the same as that of 2.1 we want to shrinkwrap a certain surface in a cover, using the boundary of that cover as a barrier surface. See Figure 3 for an idealized depiction of $T^{\prime}$ and $S$ in $W$ and $X$ in the case that $W$ is a handlebody.

Lemma 2.3 (Main construction lemma). Let $\mathcal{E}$ be an end of the complete open orientable parabolic free hyperbolic 3-manifold $N$ with finitely generated fundamental group. Let $W \subset N$ be a submanifold such that $\partial W \cap$ int $(N)$ separates $W$ from $\mathcal{E}$. Let $\Delta_{1} \subset N \backslash \partial W$ be a finite collection of simple closed geodesics with $\Delta=\operatorname{int}(W) \cap \Delta_{1}$ a nonempty proper subset of $\Delta_{1}$. Suppose further that $\partial W$ is 2-incompressible rel. $\Delta$.

Let $G$ be a finitely generated subgroup of $\pi_{1}(W)$, and let $X$ be the covering space of $W$ corresponding to $G$. Let $\Sigma$ be the preimage of $\Delta$ in $X$, and $\hat{\Delta} \subset \Sigma$ a subset which maps homeomorphically onto $\Delta$ under the covering projection, and let $B \subset \hat{\Delta}$ be a nonempty union of geodesics. Suppose there exists an embedded closed surface $S \subset X \backslash B$ that is 2-incompressible rel. $B$ in $X$, which separates every component of $B$ from $\partial X$.

Then $\partial W$ can be homotoped to a $\Delta_{1}$-minimal surface which, by abuse of notation, we call $\partial W^{\prime}$, and the map of $S$ into $N$ given by the covering projection is homotopic to a map whose image $T^{\prime}$ is $\Delta_{1}$-minimal. Also, $\partial W^{\prime}$ (resp. $T^{\prime}$ ) can be perturbed by an arbitrarily small perturbation to be an embedded (resp. smoothly immersed) 
surface $\partial W_{t}$ (resp. $T_{t}$ ) bounding $W_{t}$ with the following properties:

(1) There exists an isotopy from $\partial W$ to $\partial W_{t}$ which never crosses $\Delta_{1}$, and which induces an isotopy from $W$ to $W_{t}$, and a corresponding deformation of hyperbolic manifolds $X$ to $X_{t}$ which fixes $\Sigma$ pointwise.

(2) There exists an isotopy from $S$ to $S_{t} \subset X_{t}$ which never crosses $B$, such that $T_{t}$ is the projection of $S_{t}$ to $N$.

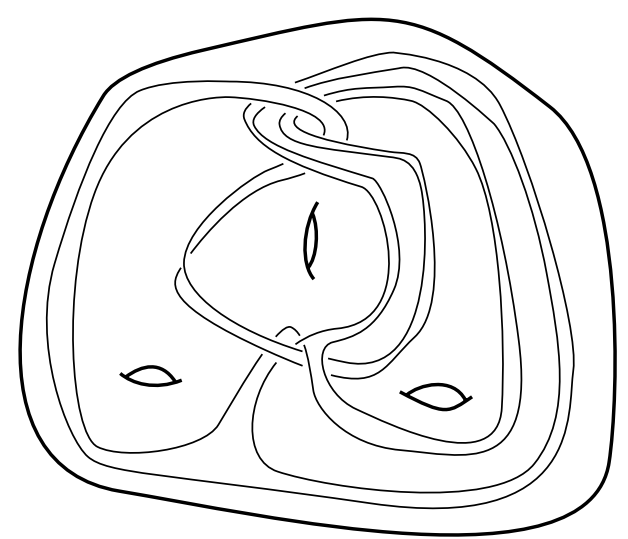

W

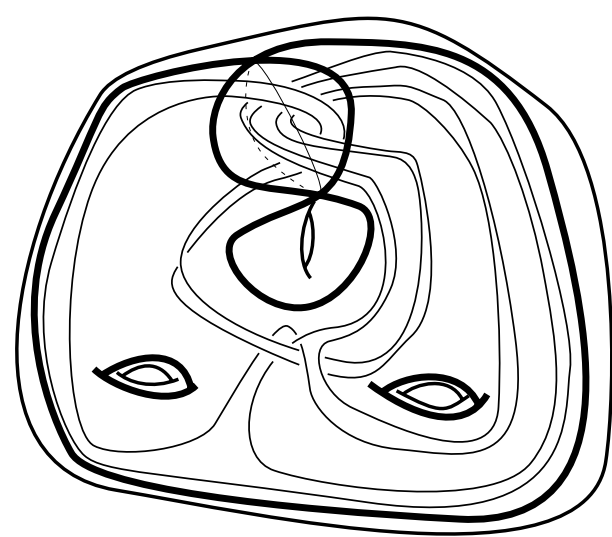

$T^{\prime} \subset W$

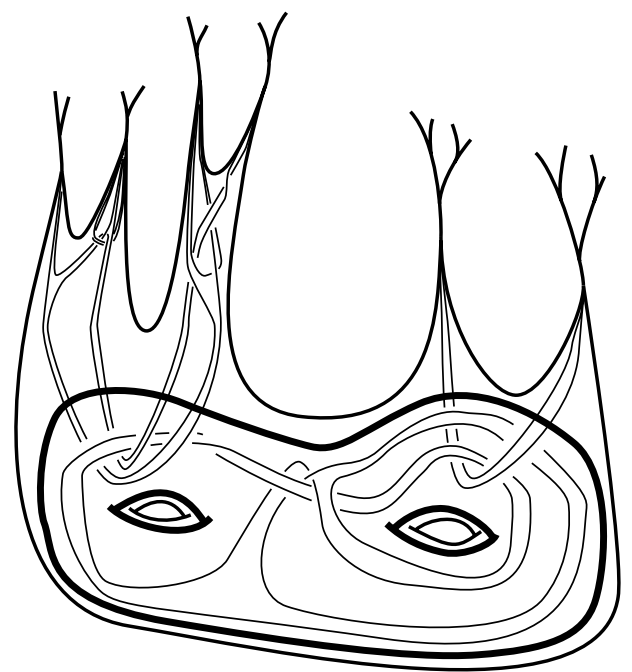

$S \subset X$

Figure 3. The surfaces $T^{\prime}$ and $S$ in $W$ and $X$ respectively

Proof. The proof is reasonably straightforward, given the work in $\$ 1$ and $\$ 2.1$. First, we obtain $\partial W^{\prime}$ from $\partial W$ by shrinkwrapping rel. $\Delta_{1}$. Since $\Delta=\operatorname{int}(W) \cap \Delta_{1}$ is a nonempty and proper subset of $\Delta_{1}, \partial W$ satisfies the hypotheses of Theorem 1.10 . and therefore $\partial W^{\prime}$ exists and satisfies the desired properties. 
For each $t=t_{i}$ in our approximating sequence, the metric $g_{t}$ on $W_{t}$ lifts to a metric on $X_{t}$ which, by abuse of notation, we also call $g_{t}$.

Then with respect to the $g_{t}$ metric, $\partial X_{t}$ acts as a barrier surface, and we can find a $g_{t}$ locally least area surface $S_{t} \subset \operatorname{int}\left(X_{t}\right)$ which is $g_{t}$ globally least area in the isotopy class of $S$ in $X_{t} \backslash N_{f(t)}(B)$, by [MSY], just as in the proof of Lemma 1.20. Note that $S$ is necessarily homologically essential in $X_{t} \backslash N_{f(t)}(B)$, since it separates each component of $B$ from $\partial X_{t}$ by hypothesis, and therefore any surface isotopic to $S$ in $X_{t} \backslash N_{f(t)}(B)$ must intersect a fixed compact arc $\alpha$ from $B$ to $\partial X_{t}$.

The immersed surfaces $T_{t} \subset N$ are obtained by mapping $S_{t}$ to $W_{t}$ by the covering projection. After passing to a further subsequence of values $t=t_{i}$, the limit of the $T_{t}$ surfaces exists as a map from $S$ to $N$, with image $T^{\prime}$, by the argument of Lemma 1.24 applied locally. The regularity of $T^{\prime}$ locally along $\Delta_{1}$ follows from the argument of Lemma 1.31, since that argument is completely local.

As in Lemma 1.34, we can repeat the construction above with the $g_{s, t}$ metrics and obtain a limit $T^{\prime}$ with the desired regularity.

It follows that $T^{\prime}$ is $\Delta_{1}$-minimal. Notice that some local sheets of $T^{\prime}$ are actually minimal (in the hyperbolic metric) near geodesics in $\Delta_{1}$, corresponding to subsets of $S_{t}$ in $X_{t}$ crossing components of $\Sigma \backslash B$, by Lemma 2.1. In any case, $T^{\prime}$ is intrinsically $\mathrm{CAT}(-1)$, and the theorem is proved.

2.3. Nonsimple geodesics. When we come to consider hyperbolic manifolds with parabolics, we need to treat the case that the geodesics $\Delta_{1}$ might not be simple. But there is a standard trick to reduce this case to the simple case, at the cost of slightly perturbing the hyperbolic metric.

Explicitly, suppose $\Gamma \subset M$ is as in the statement of Theorem 1.10 except that some of the components are possibly not simple. Then for every $\epsilon>0$ there exists a perturbation $g$ of the hyperbolic metric on $M$ in a neighborhood of $\Gamma$ with the following properties:

(1) The new metric $g$ agrees with the hyperbolic metric outside $N_{\epsilon}(\Gamma)$.

(2) With respect to the metric $g$, the curves in $\Gamma$ are homotopic to a collection of simple geodesics $\Gamma^{\prime}$.

(3) The metric $g$ is hyperbolic (i.e. has constant curvature -1 ) on $N_{\epsilon / 2}\left(\Gamma^{\prime}\right)$.

(4) The metric $g$ is $(1+\epsilon)$-bilipschitz equivalent to the hyperbolic metric, and the sectional curvature of the $g$ metric is pinched between $-1-\epsilon$ and $-1+\epsilon$.

The existence of such a metric $g$ follows from Lemma 5.5 of [Ca]. To make an orthopedic comparison: think of the nonsimple geodesics $\Gamma$ as a collection of unnaturally fused bones in $N_{\epsilon}(\Gamma)$; the bones are broken, reset, and heal as simple geodesics in the new metric.

It is clear that the methods of $\$ 1$ apply equally well to the metric $g$, and therefore shrinkwrapping can be done with respect to the metric $g$, producing a surface which is intrinsically $\operatorname{CAT}(-1+\epsilon)$.

In fact, since such a metric exists for each $\epsilon$, we can take a sequence of such metrics $g_{\epsilon}$ for each small $\epsilon>0$, produce a shrinkwrapped surface $T_{\epsilon}$ for each such $\epsilon$, and take a limit $T$ as $\epsilon \rightarrow 0$ which is intrinsically $\mathrm{CAT}(-1)$, and which can be approximated by embedded surfaces, isotopic to $S$, in the complement of $\Gamma \backslash C$, where $C$ is a finite subset of geodesics whose cardinality can be a priori bounded above in terms of the genus of $S$. We will not be using this stronger fact in the sequel, since the existence of a $\operatorname{CAT}(-1+\epsilon)$ surface is quite enough for our purposes. 


\section{Asymptotic tube RAdiUs AND LENGTH}

By $[\mathrm{BO}$ an end of a complete hyperbolic 3-manifold $N$ is geometrically infinite if and only if there exists an exiting sequence of closed geodesics. In this chapter we show that if $\pi_{1}(N)$ is parabolic free, then the geodesics can be chosen to be $\eta$-separated for some $\eta$; in particular, all are simple.

Definition 3.1. Let $N$ be a complete hyperbolic 3-manifold with geometrically infinite end $\mathcal{E}$. Define the $\mathcal{E}$-asymptotic tube radius to be the supremum over all sequences $\left\{\gamma_{i}\right\}$ of closed geodesics exiting $\mathcal{E}$, of

$$
\limsup _{i \rightarrow \infty} \text { tube } \operatorname{radius}\left(\gamma_{i}\right) \text {. }
$$

Similarly define the $\mathcal{E}$-asymptotic length to be the infimum over all sequences $\left\{\gamma_{i}\right\}$ as before of

$$
\liminf _{i \rightarrow \infty} \operatorname{length}\left(\gamma_{i}\right) .
$$

We will drop the prefix $\mathcal{E}$ when the end in question is understood.

Proposition 3.2. If $\mathcal{E}$ is a geometrically infinite end of the complete hyperbolic 3 -manifold $N$ without parabolics, then the asymptotic tube radius $>1 / 4$ asymptotic length. If asymptotic length $=0$, then the asymptotic tube radius $=\infty$. There exists a uniform lower bound $\eta=0.025$ to the asymptotic tube radius of a geometrically infinite end of a complete parabolic free hyperbolic 3-manifold.

Proof. Meyerhoff [Me defines a monotonically decreasing function $r:(0,0.1] \rightarrow$ $[0.3, \infty)$ such that if $\gamma$ is a closed geodesic in $N$ and length $(\gamma) \leq t$, then tube $\operatorname{radius}(\gamma) \geq r(t)$. Furthermore, $\lim _{t \rightarrow 0} r(t)=\infty$. Therefore, the second statement of Proposition 3.2 follows from $[\mathrm{Me}$ and the third follows from the first statement and [Me]. (Actually, Proposition 3.3 will show that $\log (3) / 2$ is a lower bound.)

Now suppose that the asymptotic length $=L \in[0.1, \infty)$. Then there exists a sequence $\left\{\gamma_{i}\right\}$ exiting $\mathcal{E}$ such that length $\left(\gamma_{i}\right) \rightarrow L$. As in [G2, §5], if tube $\operatorname{radius}\left(\gamma_{t}\right) \leq \frac{1}{4}$ length $\left(\gamma_{i}\right)$, then there exists a geodesic $\beta_{i}$ homotopic to a curve which is a union of a segment of $\gamma_{i}$ and an orthogonal arc from $\gamma_{i}$ to itself, and each of these segments has length $\leq$ length $\left(\gamma_{i}\right) / 2$. By straightening these segments and using the law of cosines, we see that if length $\left(\gamma_{i}\right) \geq 0.099$, then length $\left(\beta_{i}\right)<\operatorname{length}\left(\gamma_{i}\right)-0.02$. Thus if limsup tube radius $\left(\gamma_{i}\right)<L / 4$, there exists a sequence $\left\{\beta_{i}\right\}$ such that $\lim \inf$ length $\left(\beta_{i}\right) \leq L-0.02$ where $\beta_{i}$ is as above. Since $\infty>L,\left\{\beta_{i}\right\}$ must exit the same end as $\left\{\gamma_{i}\right\}$, which is a contradiction.

Now suppose that the asymptotic length is infinite and $\left\{\gamma_{i}\right\}$ is an exiting sequence such that length $\left(\gamma_{i}\right) \rightarrow \infty$. Given $R \geq 10$ we produce a new exiting sequence $\left\{\sigma_{i}\right\}$ with tube radius $\left(\sigma_{i}\right)>R$ for all $i$. If possible let $\alpha_{i}$ be a smallest segment of $\gamma_{i}$ such that there is a geodesic path $\beta_{i}$ connecting $\partial \alpha_{i}$, length $\left(\beta_{i}\right) \leq 10 R$ and $\beta_{i}$ is not homotopic to $\alpha_{i}$ rel endpoints. If $\alpha_{i}$ does not exist, then tube radius $\left(\gamma_{i}\right) \geq 5 R$. So let us assume that for all $i, \alpha_{i}$ exists. Note that length $\left(\alpha_{i}\right) \rightarrow \infty$ or else the concatenations $\left\{\alpha_{i} * \beta_{i}\right\}$ are homotopic to an exiting sequence of bounded length geodesics. Also, asymptotic length infinite implies that as $i \rightarrow \infty$, the injectivity radius of points of $\gamma_{i}$ (and hence $\alpha_{i} \cup \beta_{i}$ ) $\rightarrow \infty$. Therefore for $i$ sufficiently large we can assume that length $\left(\alpha_{i}\right)>10 R$, length $(\beta)=10 R$, and both of the angles between $\beta_{i}$ and $\alpha_{i}$ are at least $\pi / 2$. The geodesic $\sigma_{i}$ homotopic to the curve obtained by concatenating $\alpha_{i}$ and $\beta_{i}$ lies within distance 2 of $\alpha_{i} \cup \beta_{i}$ and for the most part lies extremely close. Indeed, if $A$ is an immersed least area annulus in $N$ with 
$\partial A=\left(\alpha_{i} * \beta_{i}\right) \cup \sigma_{i}$, then the Gauss-Bonnet formula (Lemma 1.4) implies that area $(A) \leq \pi$. Since the intrinsic curvature of $A$ is $\leq-1$, it follows that for $i$ sufficiently large, no point $a$ of $A$ can be at distance 1 from $\alpha_{i} \cup \beta_{i}$ and distance at least 1 from $\sigma_{i}$, for the area of the disc of radius 1 about $a \in A$ would be $\geq \pi$.

If tube radius $\left(\sigma_{i}\right) \leq R$, then there would be an $\operatorname{arc} \tau_{i}$ connecting points of $\sigma_{i}$ such that length $\left(\tau_{i}\right) \leq 2 R$ and $\tau_{i}$ cannot be homotoped rel endpoints into $\sigma_{i}$. Thus, for $i$ sufficiently large one finds new essential geodesic paths $\beta_{i}^{\prime}$ of length $\leq 10 R$ with endpoints in $\alpha_{i}-\partial \alpha_{i}$. This contradicts the minimality property of $\alpha_{i}$.

Proposition 3.3. If $\mathcal{E}$ is an end of the complete, orientable, hyperbolic 3-manifold $N$ and $\pi_{1}(N)$ has no parabolic elements, then the $\mathcal{E}$-asymptotic tube radius $>$ $\log (3) / 2$.

Remark 3.4. We will not be using Proposition 3.3 in this paper.

Proof. Let $\left\{\gamma_{i}\right\}$ be a sequence of geodesics exiting $\mathcal{E}$ such that

$$
\lim _{i} \operatorname{length}\left(\gamma_{i}\right)=l=\mathcal{E} \text {-asymptotic length. }
$$

If $l$ is small, i.e. $l \leq 0.978$, then for $i$ sufficiently large, tube radius $\left(\gamma_{i}\right) \geq \log (3) / 2$ by [Me as explained in GMT, Proposition 1.11]. If $l$ is large, then for $i$ sufficiently large, tube radius $\left(\gamma_{i}\right) \geq \log (3) / 2$ by Proposition 3.2. A hyperbolic geometry argument, slightly more sophisticated than the one cited above shows that $l \geq 1.289785$ suffices. Indeed, the proof of Proposition 1.11 in GMT] shows that there exists $\epsilon>0$ such that if length $\left(\gamma_{i}\right) \geq 1.289785$, then either tube $\operatorname{radius}\left(\gamma_{i}\right) \geq \log (3) / 2$ or there exists an essential closed curve $\kappa_{i}$ such that length $\left(\kappa_{i}\right) \leq \operatorname{length}\left(\gamma_{i}\right)-\epsilon$ and $d\left(\gamma_{i}, \kappa_{i}\right) \leq l$. If $\kappa_{i}^{*}$ denotes the geodesic homotopic to $\kappa_{i}$, then $\left\{\kappa_{i}^{*}\right\}$ exits $\mathcal{E}$ and $\lim \inf \operatorname{length}\left(\kappa_{i}^{*}\right) \leq l-\epsilon$, which contradicts the fact that $l$ is asymptotic length.

It follows from [GMT, JR, Li and CLLM] that if $\delta$ is a shortest geodesic in a complete hyperbolic 3-manifold $N$, then either tube radius $\delta \geq \log (3) / 2$ or $N$ is a closed hyperbolic 3-manifold. (See Conjecture 1.31 in [GMT].) Therefore, if each $\gamma_{i}$ is a shortest length geodesic in $N$, then the proof of Proposition 3.3 follows.

Assuming that asymptotic tube radius $<\log (3) / 2$, we will derive a contradiction using techniques which require an understanding of [GMT, §1]. Nevertheless, the punch line follows exactly as in two paragraphs above. Here is the idea. Associated to each $\gamma_{i}$ there is a 2-generator subgroup of $\pi_{1}(N)$ defined as follows. When viewed as acting on $\mathbb{H}^{3}$, one generator $f_{i}$ is a shortest translation along a lift of $\gamma_{i}$ and the other generator $w_{i}$ takes that lift to a nearest translate. After passing to a subsequence, there exists $\epsilon>0, K<\infty$ such that for each $i$, there exists a closed curve $\kappa_{i}$ such that $d\left(\kappa_{i}, \gamma_{i}\right) \leq K$ and length $\left(\kappa_{i}\right)<\operatorname{length} \gamma_{i}-\epsilon$. Here $\kappa_{i}$ represents an element in the group generated by $f_{i}$ and $w_{i}$.

Here are the details. Given $\left\{\gamma_{i}\right\}$ there exist sequences $\left\{A_{i}\right\},\left\{A_{i}^{\prime}\right\}$ where $A_{i}$ is a lift of $\gamma_{i}$ to $\mathbb{H}^{3}$ and $A_{i}^{\prime}=w_{i}(A)$ is a nearest $\pi_{1}(N)$ translate of $A$, where $w_{i} \in \pi_{1}(N)$. By Definition 1.8 in GMT associated to $f_{i}$ and $w_{i}$ there is a triple of complex numbers $\left(L_{i}, D_{i}, R_{i}\right)$ where length $\left(f_{i}\right)=\operatorname{Re}\left(L_{i}\right)$ and $\operatorname{Re}\left(D_{i}\right)=d\left(A, A^{\prime}\right)$. By compactness, after passing to a subsequence, $\left\{\left(L_{i}, D_{i}, R_{i}\right)\right\}$ converges to $(L, D, R)$, where $\operatorname{Re}(L)=$ l. Again by Definition 1.8, $(L, D, R)$ gives rise to a marked 2-generator group $\langle f, w\rangle$ where $f_{i} \rightarrow f$ and $w_{i} \rightarrow w$. By Lemma 1.13 in [GMT] we can assume that $(L, D, R)$ and the various $\left(L_{i}, D_{i}, R_{i}\right)$ lie in the parameter space $\mathcal{P}$ defined in [GMT, 1.11]. It cannot lie in one of the 7 exceptional regions given in Table 1.2 of [GMT, or else by [GMT, Chapter 3], [JR], [Li] and CLLM], it and $\left(L_{i}, D_{i}, R_{i}\right)$ correspond to a closed 
hyperbolic 3-manifold for $i$ sufficiently large, for it is shown in these papers that a neighborhood of each exceptional region corresponds to a unique closed hyperbolic 3 -manifold as conjectured in GMT, 1.31]. This implies that $N$ is covered by a closed 3-manifold, which is a contradiction.

The proof of Proposition 1.28 in [GMT] shows that if $(L, D, R)$ does not lie in an exceptional region, then there exists a killer word $u(f, w)$ in $f$ and $w$ as defined in GMT, 1.18]. This means that length $(u(f, w))<\operatorname{length}(f)$ or if $A=\operatorname{axis}(f)$, then $d(A, u(f, w) A)<\operatorname{Re}(D)=d(A, w(A))$. Therefore, for $i$ sufficiently large, either length $\left(u\left(f_{i}, w_{i}\right)\right)<\operatorname{length}\left(f_{i}\right)$ or $d\left(A_{i}, u\left(f_{i}, w_{i}\right) A_{i}\right)<\operatorname{Re}\left(D_{i}\right)$. The latter cannot happen since $w_{i}$ was chosen to take $A_{i}$ to a nearest translate.

Since the nonexceptional points of $\mathcal{P}$ are covered by finitely many compact regions and each region has a killer word, it follows that for a correct choice of killer words, reduction of length is uniformly bounded below by some constant $\epsilon$.

Since $\pi_{1}(N)$ has no parabolics, $u\left(f_{i}, w_{i}\right)$ corresponds to a hyperbolic element and hence a geodesic $\sigma_{i} \subset N$. If $u(f, w)$ is loxodromic, then the corresponding geodesic $\tilde{\sigma} \subset \mathbb{H}^{3}$ is of bounded distance from $A$. Therefore, for all $i, d\left(\sigma_{i}, A_{i}\right)$ is uniformly bounded and hence $\left\{\sigma_{i}\right\}$ exits $\mathcal{E}$. Thus, asymptotic length $\leq l-\epsilon$, which is a contradiction. If $u(f, w)$ is parabolic, then length $\left(\sigma_{i}\right) \rightarrow 0,\left\{\sigma_{i}\right\}$ exits the same end as $\left\{\gamma_{i}\right\}$ and hence asymptotic length equals zero. To see that $\left\{\sigma_{i}\right\}$ exits $\mathcal{E}$, note that in $\mathbb{H}^{3}, u(f, w)$ takes a point $x$ to $y$ where $d(x, y)<l / 4$ and hence, for $i$ sufficiently large, there are essential closed curves of length $<l / 4$ passing within $2 d(x, A)$ from $\gamma_{i}$.

Question 3.5. What is the maximal lower bound for the asymptotic tube radius of a geometrically infinite end $\mathcal{E}$ of a complete, orientable, hyperbolic manifold with finitely generated fundamental group, both in the cases that $\mathcal{E}$ is parabolic free or not?

Question 3.6. What is the upper bound for asymptotic length of a geometrically infinite end $\mathcal{E}$ ? It follows from Theorem 0.9 that there is an upper bound which is a function of $\operatorname{rank}\left(\pi_{1}(\mathcal{E})\right)$.

\section{Canary's Theorem}

In this section we give a proof of Canary's theorem (Theorem 4.1) when $N$ is parabolic free. Our proof of Theorem 0.9 will closely parallel this argument.

Theorem 4.1 (Canary). If $\mathcal{E}$ is a topologically tame end of the complete, orientable, hyperbolic 3-manifold $N=\mathbb{H}^{3} / \Gamma$, where $\Gamma$ has no parabolic elements, then either $\mathcal{E}$ is geometrically finite or there exists a sequence of $C A T(-1)$ surfaces exiting the end. If $\mathcal{E}$ is parametrized by $S \times[0, \infty)$, then these surfaces are homotopic to surfaces of the form $S \times t$, via a homotopy supported in $S \times[0, \infty)$.

Proof. It suffices to consider the case that $\mathcal{E}$ is geometrically infinite. By Proposition 3.2 there exists a sequence of pairwise disjoint $\eta$-separated simple closed geodesics $\Delta=\left\{\delta_{i}\right\}$ exiting $\mathcal{E}$. Assume that $\Delta$ and the parametrization of $\mathcal{E}$ are chosen so that for all $i \in \mathbb{N}, \delta_{i} \subset S \times(i-1, i)$. Let $g=\operatorname{genus}(S), \Delta_{i}=\left\{\delta_{1}, \cdots, \delta_{i}\right\}$ and let $\left\{\alpha_{i}\right\}$ be a locally finite collection of embedded proper rays in $\mathcal{E}$ such that $\partial \alpha_{i} \in \delta_{i}$.

An idea used repeatedly, in various guises, throughout this paper is the following. If $R$ is a closed oriented surface and $T$ is obtained by shrinkwrapping $R$ rel the 
geodesics $\Delta_{R}$, then $R$ is homotopic to $T$ via a homotopy which does not meet $\Delta_{R}$, except possibly at the last instant. Therefore, if $\delta_{i} \subset \Delta_{R}$ and $\left\langle R, \alpha_{i}\right\rangle=1$, then $T \cap \alpha_{i} \neq \emptyset$ and if $T \cap \delta_{i}=\emptyset$, then $\left\langle T, \alpha_{i}\right\rangle=1$. Here $\langle\cdot, \cdot\rangle$ denotes the algebraic intersection number.

Warm-up Case. Each $S \times i$ is 2-incompressible in $N \backslash \Delta$. (E.g. $N=S \times \mathbb{R}$.)

Proof. Apply Theorem 0.8 to shrinkwrap $S \times i$ rel $\Delta_{i+1}$ to a $\mathrm{CAT}(-1)$ surface $S_{i}$. Since $\left\langle S \times i, \alpha_{i}\right\rangle=1, S_{i} \cap \alpha_{i} \neq \emptyset$. Since $\left\{\alpha_{i}\right\}$ is locally finite, the Bounded Diameter Lemma implies that the $S_{i}$ 's must exit $\mathcal{E}$. Therefore for $i$ sufficiently large, $S_{i} \subset \mathcal{E}$ and $\left\langle S_{i}, \alpha_{1}\right\rangle=1$; hence the projection of $S_{i}$ into $S \times 0$ (given by the product structure on $\mathcal{E}$ ) is a degree-1 map between surfaces of the same genus. Since such maps are homotopic to homeomorphisms, we see that $S_{i}$ can be homotoped within $\mathcal{E}$ to a homeomorphism onto $S \times 0$. See Figure 4 for a schematic view.

Q: How can one find an exiting sequence of $\mathrm{CAT}(-1)$ surfaces?

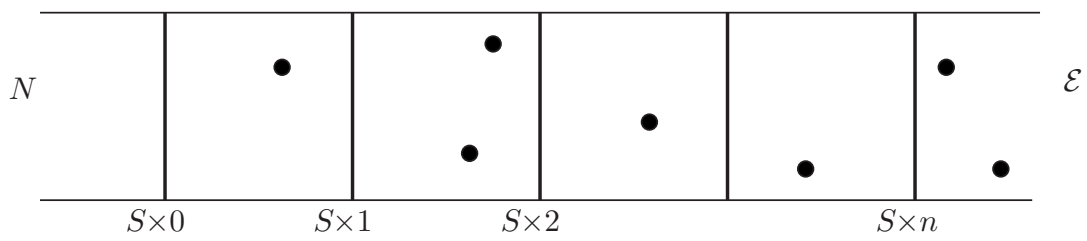

A: Shrinkwrap!

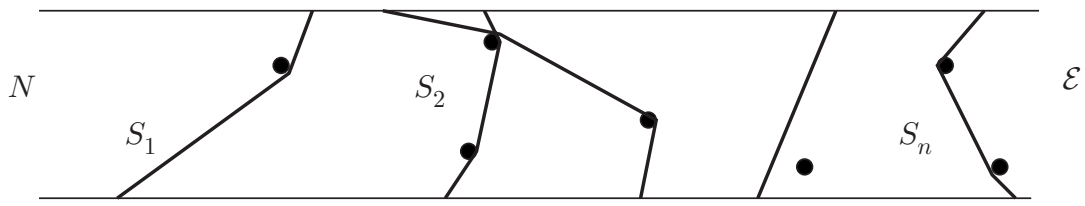

Figure 4. A schematic depiction of shrinkwrapping in action

General Case. (E.g. $N$ is an open handlebody.)

Proof. Without loss of generality we can assume that every closed orientable surface separates $N$ (see Lemma 5.1 and Lemma 5.6). We use a purely combinatorial/topological argument to find a particular sequence of smooth surfaces exiting $\mathcal{E}$. We then shrinkwrap these surfaces and show that they have the desired escaping and homological properties.

Fix $i$. If possible, compress $S \times i$ via a compression which either misses $\Delta$ or crosses $\Delta$ once say at $\delta_{i_{1}} \subset \Delta_{i}$. If possible, compress again via a compression meeting $\Delta \backslash \delta_{i_{1}}$ at most once say at $\delta_{i_{2}} \subset \Delta_{i}$. After at most $n \leq 2 g-2$ such operations and deleting 2 -spheres we obtain embedded connected surfaces $S_{1}^{i}, \cdots, S_{i_{r}}^{i}$, none of which is a 2-sphere and each of which is 2-incompressible rel $\Delta_{i+1} \backslash\left\{\delta_{i_{1}} \cup \cdots \cup \delta_{i_{n}}\right\}$. For each fixed $i$, each $\delta_{j}(j \leq i)$ with at most $2 g-2$ exceptions is separated from 
$\mathcal{E}$ by exactly one surface $S_{k}^{i}$. Call $B a g_{k}^{i}$ the region separated from $\mathcal{E}$ by $S_{k}^{i}$. Note that all compressions in the passage of $S_{i}$ to $\left\{S_{1}^{i}, \cdots, S_{i_{r}}^{i}\right\}$ are on the non- $\mathcal{E}$-side.

Since each $i_{r} \leq g$, we can find a $p \in \mathbb{N}$ and for each $i$, a reordering of the $S_{j}^{i}$ 's (and their bags) so that for infinitely many $i \geq p, \delta_{p} \in \mathrm{Bag}_{1}^{i}$; furthermore, if for each $i$ such that $\delta_{p} \in \operatorname{Bag}_{1}^{i}$, we denote by $p(i)$ the maximal index such that $\delta_{p(i)} \in \mathrm{Bag}_{1}^{i}$, then the set $\{p(i)\}$ is unbounded. By Theorem 0.8 , $S_{1}^{i}$ is homotopic rel $\Delta_{i+1} \backslash\left\{\delta_{i_{1}}, \cdots, \delta_{i_{n}}\right\}$ to a CAT $(-1)$ surface $S_{i}$. Since the collection $\left\{\alpha_{p(i)}\right\}$ is infinite and locally finite, the Bounded Diameter Lemma implies that a subsequence of these $S_{1}^{i}$ 's must exit $\mathcal{E}$. Call this subsequence $T_{1}, T_{2}, \cdots$, where $T_{i}$ is the shrinkwrapped $S_{1}^{n_{i}}$. Therefore, for $i$ sufficiently large, $T_{i}$ must lie in $S \times(p, \infty)$ and $\left\langle T_{i}, \alpha_{p}\right\rangle=$ $\left\langle S_{1}^{n_{i}}, \alpha_{p},\right\rangle=1$. Therefore, projection of $T_{i}$ to $S \times p$ is degree 1 . This in turn implies that genus $T_{i}=g$ and $T_{i}$ can be homotoped within $\mathcal{E}$ to a homeomorphism onto $S \times 0$. See Figure 5 for another schematic view.

Remark 4.2. This argument shows that for $i$ sufficiently large, $S \times i$ is already 2incompressible in $N \backslash \Delta_{i}$. Also, given any $\eta$-separated collection of exiting geodesics a sufficiently large finite subset is 2-disc busting. Actually, using the technology of the last chapter, this statement holds for any sequence of exiting closed geodesics.

The proof of Theorem 0.9 follows a similar strategy. Here is the outline in the case that $N$ has a single end $\mathcal{E}$ and no parabolics. Given a sequence of $\eta$-separated exiting simple closed geodesics $\Delta=\left\{\delta_{i}\right\}$ we pass to a subsequence (and possibly choose $\delta_{1}$ to have finitely many components) and find a sequence of connected embedded surfaces denoted $\left\{\partial W_{i}\right\}$ such that for each $i, \partial W_{i}$ separates $\Delta_{i}=\delta_{1} \cup \delta_{2} \cup \cdots \cup \delta_{i}$ from $\Delta-\Delta_{i}$ and is 2-incompressible rel $\Delta_{i}$. It is a priori possible that the $\partial W_{i}$ 's do not exit $\mathcal{E}$. If $W_{i}$ denotes the compact region split off by $\partial W_{i}$, then after possibly deleting an initial finite set of $W_{i}$ 's (and adding the associated $\delta_{i}$ 's to $\delta_{1}$ ) we find a compact 3-manifold $D \subset W_{1}$ which is a core for $\mathcal{W}=\bigcup W_{i}$.

We next find an immersed genus $\leq g$ surface $T_{i}$, which homologically separates off a subset $B_{i}$ of $\Delta_{i}$ from $\mathcal{E}$. For infinitely many $i, B_{i}$ includes a fixed $\delta_{p}$ and for these $i$ 's the set $\{p(i)\}$ is unbounded, where $p(i)$ is the largest index of a $\delta_{k} \subset B_{i}$. The surface $T_{i}$ separates $B_{i}$ from the rest in the sense that $T_{i}$ lifts to an embedded surface $\hat{T}_{i}$ in the $\pi_{1}(D)$-cover $\hat{W}_{i}$ of $W_{i}$ and in that cover $\hat{T}_{i}$ separates a lift $\hat{B}_{i}$ from $\partial \hat{W}_{i}$, the preimage of $\partial W_{i}$. The argument to this point is purely topological and applies to any 3-manifold with finitely generated fundamental group. In the general case, $\left\{\partial W_{i}\right\}$ will not be an exiting sequence.

Next we shrinkwrap $\partial W_{i}$ rel $\Delta_{i+1}$ to a CAT $(-1)$ surface which we continue to call $\partial W_{i}$. Then we homotope $\hat{T}_{i}$ rel $\hat{\Delta}_{i}$ to a CAT(-1) surface in the induced $\hat{W}_{i}$ and let $T_{i}$ denote the projection of $T_{i}$ to $N$. The point of shrinkwrapping $\partial W_{i}$ is that $\partial \hat{W}_{i}$ is now a barrier which prevents $\hat{T}_{i}$ from popping out of $\hat{W}_{i}$ during the subsequent shrinkwrapping (compare with $\S 2$ ). We use the $\delta_{p(i)}$ 's to show that, after passing to a subsequence, the $T_{i}$ 's exit $\mathcal{E}$. We use $\delta_{p}$ to show that for $i$ sufficiently large, $T_{i}$ homologically separates $\mathcal{E}$ from a Scott core of $N$.

We have outlined the strategy. For purposes of exposition, the above sketch of the construction of the $T_{i}$ 's is slightly different from that given in $\S 6$.

In $\$ 7$ we make the necessary embellishments to handle the parabolic case.

The next chapter develops the theory of end reductions which enables us to define the submanifolds $W_{i}$. 

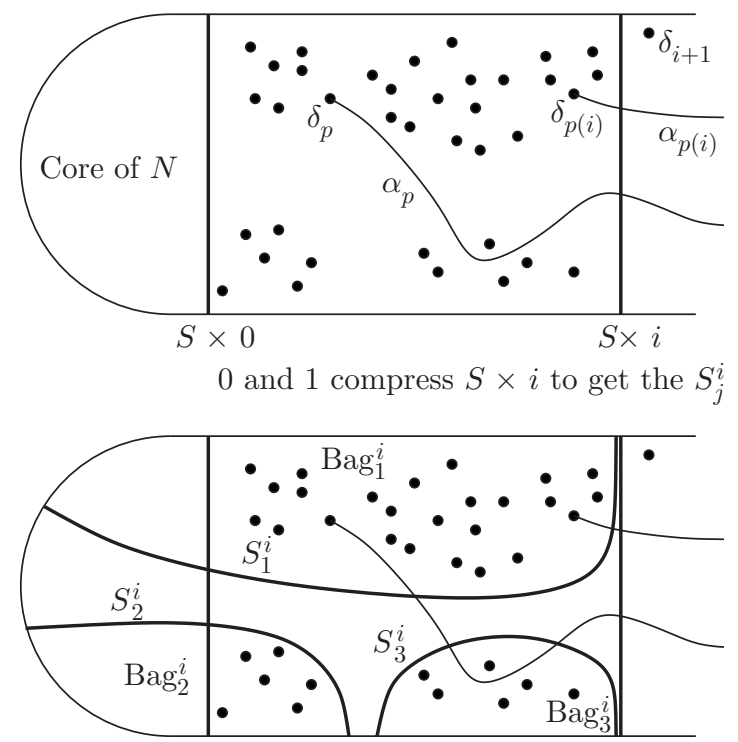

Shrinkwrap $S_{1}^{i}$ to get $T_{i}$

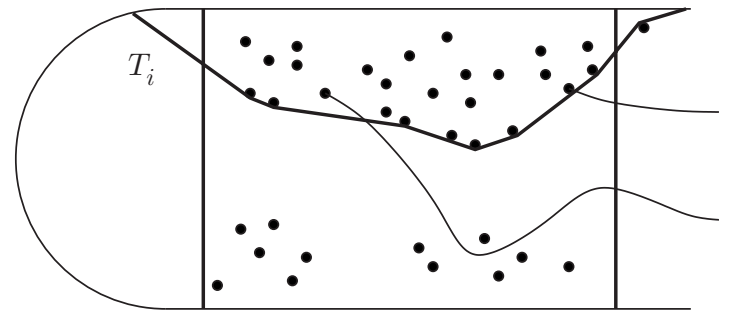

Actual picture

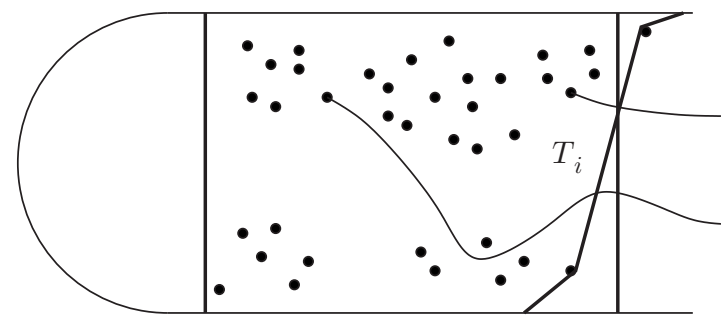

Figure 5. The Bounded Diameter Lemma and the intersection number argument show that for $i$ sufficiently large, $S \times i$ undergoes no compression, and $T_{i}$ actually separates all of $\Delta_{i}$ from $\mathcal{E}$.

\section{END MANIFOLDS AND END REDUCTIONS}

In this section, we prove a structure theorem for the topology of an end of a 3-manifold with finitely generated fundamental group. A reference for basic 3manifold topology is $\mathrm{He}$.

The first step is to replace our original manifold with a 1-ended manifold $M$ with the homotopy type of a bouquet of circles and closed orientable surfaces. We 
then prove Theorem 5.21, the infinite end engulfing theorem, which says that given an exiting sequence of homotopically nontrivial simple closed curves we can pass to a subsequence $\Gamma$ and find a submanifold $\mathcal{W}$, with finitely generated fundamental group containing $\Gamma$, which has the following properties:

(1) $\mathcal{W}$ can be exhausted by codimension- 0 compact submanifolds $W_{i}$ whose boundaries are 2-incompressible rel $\Gamma \cap W_{i}$.

(2) $\mathcal{W}$ has a core which lies in $W_{1}$.

This completes the preliminary step in the proof of Theorem 0.9, as explained at the end of $\S 4$. The proof of Theorem 0.9 itself is in $\S 6$.

In what follows we will assume that all 3-manifolds are orientable and irreducible.

Lemma 5.1. If $\mathcal{E}$ is an end of an open Riemannian 3-manifold $M^{\prime}$ with finitely generated fundamental group, then $\mathcal{E}$ is isometric to the end of a 1-ended 3-manifold $M$ whose (possibly empty) boundary is a finite union of closed orientable surfaces. A core of $M$ is obtained by attaching 1-handles to the components of $\partial M$, unless $\partial M=\emptyset$, in which case a core is a 1-complex and $M=M^{\prime}$.

Proof. A thickened Scott core $C\left[\mathrm{Sc}\right.$ of $M^{\prime}$ is a union of 1-handles (possibly empty) attached to a compact 3-manifold $X$ with incompressible boundary. Split $M^{\prime}$ along all the boundary components of $X$ and let $M$ be the component which contains $\mathcal{E}$.

Remark 5.2. $M$ is a submanifold of $M^{\prime} . M$ is isometric to a submanifold $\hat{M}$ of the covering of $M^{\prime}$ corresponding to the inclusion $\pi_{1}(M) \rightarrow \pi_{1}\left(M^{\prime}\right)$, and the inclusion $M \rightarrow \hat{M}$ is a homotopy equivalence.

Definition 5.3. Call a finitely generated group a free/surface group if it is a free product of orientable surface groups and a free group. Call a 1-ended, irreducible, orientable, 3-manifold $M$ an end-manifold if it has a compact (possibly empty) boundary and a compact core of the form $\partial M \times I \cup 1$-handles if $\partial M \neq \emptyset$ or a handlebody if $\partial M=\emptyset$.

Note that $\pi_{1}(M)$ is a free/surface group for $M$ an end-manifold.

Lemma 5.4. If $G$ is a subgroup of a free/surface group, then its $\pi_{1}$-rank equals its $H_{1}$-rank, both in $\mathbb{Z}$ and $\mathbb{Z} / 2 \mathbb{Z}$-coefficients.

Proof. A finitely generated subgroup of a free/surface group is a free/surface group, and equality holds in that case. An infinitely generated subgroup of a free/surface group contains an infinitely generated free summand. Consequently, both the $\pi_{1^{-}}$ rank and the $H_{1}$-rank are infinite for such subgroups.

Lemma 5.5. An $H_{1}$-injective subgroup $G$ of a free/surface group $K$ is finitely generated.

Proof. Rank $\pi_{1}(G)=\operatorname{rank}(G /[G, G]) \leq \operatorname{rank}(K /[K, K])=\operatorname{rank} \pi_{1}(K)<\infty$.

Lemma 5.6. A 1-ended, orientable, irreducible 3-manifold $M$ with compact boundary is an end-manifold if and only if $\pi_{1}(M)$ is a free/surface group, $H_{2}(M, \partial M)=0$ and $\partial M$ is $\pi_{1}$-injective.

Every closed embedded $\pi_{1}$-injective surface in an end-manifold is boundary parallel. 
Proof. Let $M$ be an end-manifold with core $C$ of the form $\partial M \times I \cup 1$-handles or handlebody if $\partial M=\emptyset$. Since the inclusion $C \rightarrow M$ is a homotopy equivalence, $\partial M$ is incompressible and $\pi_{1}(M)$ is a free/surface group. If $T \subset M$ is a compact properly embedded $\pi_{1}$-injective surface, then $T$ can be homotoped rel $\partial T$ into $C$. The cocores $D_{i}$ of the 1-handles are properly embedded disks whose boundary misses $\partial T$. Since $T$ is homotopically essential, it follows that each intersection $T \cap D_{i}$ is homotopically inessential in $T$, and therefore $T$ can be homotoped off the cocores of the 1-handles. Once this is done, $T$ can be further homotoped rel. boundary into $\partial M$, since $C$ deformation retracts to $\partial M$ in the complement of the cocores of the 1-handles. This implies that $H_{2}(M, \partial M)=0$.

If $H_{2}(M, \partial M)=0$ and $M$ has incompressible boundary, a connected, closed orientable incompressible surface $R$ must separate off a connected, compact Haken manifold $X$ with incompressible boundary. If $\pi_{1}(M)$ is also a free/surface group, then $\pi_{1}(X)$ is a closed orientable surface group and using [St] we conclude that $X=N(T)$ for some component $T$ of $\partial M$, so $R$ is boundary parallel. Therefore, if $\partial M=\emptyset$, then any core is a handlebody. If $\partial M \neq \emptyset$, then $M$ has a core $C$ which contains $\partial M\left[\underline{\mathrm{Mc}}\right.$. If $C^{\prime}$ is obtained by maximally compressing $C$, then each component of $\partial C^{\prime}$ is boundary parallel and hence $C=\partial M \times I \cup 1$-handles.

Corollary 5.7. If $\mathcal{W}$ is a 1-ended, $\pi_{1}$-injective submanifold of the end-manifold $M$ such that $\pi_{1}(\mathcal{W})$ is finitely generated and $\partial \mathcal{W}$ is a union of components of $\partial M$, then $\mathcal{W}$ is an end-manifold.

Definition 5.8. Given a connected compact subset $J$ of an open irreducible 3manifold $M$, the end-reduction $\mathcal{W}_{J}$ of $J$ to $M$ is to first approximation the smallest open submanifold of $M$ which can engulf, up to isotopy, any closed surface in $M \backslash J$ which is incompressible in $M \backslash J$. End-reductions were introduced by Brin and Thickstun BT1, BT2. Their basic properties were developed by Brin and Thickstun BT1, BT2 and Myers $\mathrm{My}$. In particular BT1 shows that $W_{J}$ can be created via the following procedure. If $V_{1} \subset V_{2} \subset \cdots$ is an exhaustion of $M$ by compact connected codimension- 0 submanifolds such that $J \subset V_{1}$, then one inductively obtains an exhaustion $W_{1} \subset W_{2} \subset \cdots$ of $\mathcal{W}_{J}$ by compact sets as follows. Transform $V_{1}$ to $W_{1}$ through a maximal series of intermediate manifolds $U_{1}=V_{1}, U_{2}, \cdots, U_{n}=W_{1}$ where $U_{k+1}$ is obtained from $U_{k}$ by one of the following 3 operations.

(1) Compress along a disc disjoint from $J$.

(2) Attach a 2-handle to $U_{k}$ which lies in $M \backslash \operatorname{int}\left(U_{k}\right)$, and whose attaching core circle is essential in $\partial U_{k}$.

(3) Delete a component of $U_{k}$ disjoint from $J$.

Having constructed $W_{i}$, pass to a subsequence of the $V_{j}$ 's and reorder so that $W_{i} \subset \operatorname{int}\left(V_{i+1}\right)$. Finally pass from $V_{i+1}$ to $W_{i+1}$ via a maximal sequence of the above operations. Since $\partial W_{i}$ is incompressible in $M-J$, an essential compression of $U_{k}$ can be isotoped rel boundary to one missing $W_{i}$. Therefore, we will assume such operations miss $W_{i}$ and hence $W_{i} \subset \operatorname{int}\left(W_{i+1}\right)$. Brin and Thickstun [BT1] show that $\mathcal{W}_{J}$ is up to isotopy independent of all choices.

We say that $\left\{W_{i}\right\}$ is a standard exhaustion of $\mathcal{W}_{J}$ if $W_{1} \subset W_{2} \subset \cdots$ and $\mathcal{W}_{J}=\bigcup_{i} W_{i}$, where for each $i, W_{i}$ arises from $V_{i}$ via a sequence of the three end-reduction operations and $V_{1} \subset V_{2} \subset \cdots$ is an exhaustion of $M$ by compact submanifolds. 
Remark 5.9. Note that operations (1) and (2) reduce the sum of the ranks of $\pi_{1}$ of the boundary components. It follows that the transition from $V_{i}$ to $W_{i}$ is obtained by a finite sequence of operations.

Remark 5.10. (Historical Note) Brin and Thickstun [BT1, [BT2] study end reductions to develop a necessary and a sufficient condition, end 1-movability, for taming an end of a 3-manifold. More recently, Myers $\overline{M y}$ has promoted the use of end reductions to address both the $\mathbb{R}^{3}$-covering space conjecture and the Marden conjecture.

Lemma 5.11. The inclusion $i_{J}: \mathcal{W}_{J} \rightarrow N$ induces $\pi_{1}$ and $H_{1}$-injections, the latter in both $\mathbb{Z}$ and $\mathbb{Z} / 2 \mathbb{Z}$ homology.

Proof. The $\pi_{1}$-injectivity was first proven in [BT2] and rediscovered in $\mathrm{My}$. Our proof of $H_{1}$-injectivity mimics the proof of $\pi_{1}$-injectivity in $\mathrm{My}$. Suppose $C \subset W_{i}$ is a union of oriented simple closed curves bounding the surface $S \subset M$. Note that by elementary 3-manifold topology, we can assume $S$ is embedded.

By choosing $n$ sufficiently large we can assume that $W_{i} \cup S \subset V_{n}$. If $V_{n}^{1}$ is obtained by adding a 2-handle to $V_{n}$, then $S \subset V_{n}^{1}$. If $V_{n}^{1}$ is obtained by compressing $V_{1}$, via a compression missing $J$, then by modifying $S$ near the compressing disc we obtain a surface $S_{1}$ spanning $C$ (orientably, if need be) with $S_{1} \subset V_{n}^{1}$. If $V_{n}^{1}$ is obtained by deleting components of $V_{1}$ which miss $C$, then $S_{1}=S \cap V_{n}^{1}$ still spans $C$. Since $W_{n}$ is obtained from $V_{n}$ by a finite sequence of such operations it follows that $C$ bounds in $W_{n}$ and hence in $\mathcal{W}_{J}$.

$H_{1}$-injectivity of $\mathcal{W}_{J}$ in $N$ gives us the following crucial corollary:

Corollary 5.12. An end-reduction in an end-manifold has finitely generated fundamental group.

Proof. Combine Lemma 5.11 with Lemma 5.5.

Definition 5.13. If $\mathcal{W}_{J}$ is an end-reduction of the codimension- 0 submanifold $J$ in $N$, then we say that $\mathcal{W}_{J}$ is trivial if $\mathcal{W}_{J}$ is isotopic to an open regular neighborhood of $J$ or equivalently $\mathcal{W}_{J}$ is isotopic to $\operatorname{int}(J) . \mathcal{W}_{J}$ is eventually trivial if it has an exhaustion $W_{1} \subset W_{2} \subset \cdots$ such that $\partial W_{i}$ is parallel to $\partial W_{j}$ for all $i, j$.

We now study end-reductions of disconnected spaces $J$. While the following technology and definitions can be given for more general objects we restrict our attention to finite unions of pairwise disjoint closed (possibly nonsimple) curves none of which lie in a 3-cell. Ultimately we will address end-reductions of infinite sequences of exiting curves.

Definition 5.14. If $J$ is a finite union of pairwise disjoint closed curves in an open irreducible 3-manifold $M$, we say that $J$ is end-nonseparable if there is a compact connected submanifold $H$ such that $J \subset \operatorname{int}(H)$ and $\partial H$ is incompressible in $M \backslash J$. Such an $H$ is called a house of $J$. If $J$ is end-nonseparable, then define $\mathcal{W}_{J}$ to be an end-reduction of $H$, and call $\mathcal{W}_{J}$ the end-reduction of $J$.

Lemma 5.15. The end-reduction $\mathcal{W}_{J}$ of an end-nonseparable union $J$ of closed curves is well defined up to isotopy.

Proof. Let $H$ and $H^{\prime}$ be two houses for $J$. We want to show that if $\mathcal{W}_{H}$ is an end-reduction of $H$, then there is an isotopy of $H^{\prime}$ to $H_{1}^{\prime}$ fixing $J$, so that the 
end-reduction $\mathcal{W}_{H_{1}^{\prime}}$ of $H_{1}^{\prime}$ is equal to $\mathcal{W}_{H}$. By the definition of a house for $J$, both $H$ and $H_{1}^{\prime}$ satisfy the property that they are connected submanifolds of $M$ whose boundaries are incompressible in $M \backslash J$.

Let $\left\{W_{i}\right\}$ be a standard exhaustion of $\mathcal{W}_{H}$ arising from the exhaustion $\left\{V_{i}\right\}$ of $M$. By passing to a subsequence we can assume that $H^{\prime} \cup H \subset V_{2}$. By considering the passage of $V_{2}$ to $W_{2}$, we observe that $H^{\prime}$ can be isotoped to $H_{1}^{\prime}$ rel $J$ to lie in $\operatorname{int}\left(W_{2}\right)$ and that $\partial W_{2}$ is incompressible in $M \backslash H_{1}^{\prime}$. Thus $\mathcal{W}_{H}$ is also an endreduction of $H^{\prime}$. Since end-reductions are unique up to isotopy the result follows, and we may unambiguously denote $\mathcal{W}_{H}$ by $\mathcal{W}_{J}$.

Lemma 5.16. Let $A$ be a finite union of pairwise disjoint closed curves in the open irreducible 3-manifold $M$. Then A canonically decomposes into finitely many maximal pairwise disjoint end-nonseparable subsets $A_{1}, \cdots, A_{n}$. Indeed, if $B$ is a maximal end-nonseparable subset of $A$, then $B=A_{i}$ for some $i$.

Proof. Since each element of $A$ is end-nonseparable, it suffices to show that if $B$ and $C$ are end-nonseparable subsets of $A$, then either $C \cup B$ is end-nonseparable or $C \cap B=\emptyset$. Let $H_{B}$ and $H_{C}$ be houses for $B$ and $C$ respectively. Let $V \subset N$ be a compact submanifold containing $H_{B} \cup H_{C}$. By considering the passage of $V$ to $W$ by a maximal sequence of compressions, 2-handle additions, and deletions which are taken with respect to $B \cup C$, one sees that $H_{B}$ (resp. $H_{C}$ ) can be isotoped to lie in $W$ via an isotopy fixing $B$ (resp. $C$ ). If $B \cap C \neq \emptyset$, then $W$ is connected and hence is a house for $B \cup C$.

Lemma 5.17. If $A_{1}, \cdots A_{n}$ are the maximal end-nonseparable components of a finite set $A$ of pairwise disjoint closed curves in an open irreducible 3-manifold $M$, then they have pairwise disjoint end-reductions. In particular they have pairwise disjoint houses.

Proof. Let $A_{1}, A_{2}, \cdots, A_{n}$ be the maximal end-nonseparable subsets of $A$. Let $\left\{V_{k}\right\}$ be an exhaustion of $M$ with $A \subset V_{1}$. Consider a sequence $V_{1}=U_{1}, \cdots, U_{n}=W_{1}$ where the passage from one to the next is isotopy, compression, 2-handle addition or deletion, where the compressions or deletions are taken with respect to $A$. By passing to a subsequence of the exhaustion we can assume that $W_{1} \subset V_{2}$, and in the above manner pass from $V_{2}$ to $W_{2}$. In like manner construct $W_{3}, W_{4}, \cdots$. By deleting finitely many of the first $W_{i}$ 's from the sequence and reindexing, we can assume that all the $W_{i}$ 's have the same number of components.

It suffices to show that if $W$ is a component of $W_{k}$, then $W$ contains a unique $A_{i}$ and that $\partial W$ is incompressible in $M \backslash A_{i}$. Indeed, it suffices to prove incompressibility of $\partial W$ in $M \backslash(W \cap A)$, for then $W$ is a house and can only contain one $A_{i}$ by maximality. If $\partial W$ is compressible in $M \backslash(W \cap A)$ it must compress to the outside via some compressing disc $D$. Consider a term $V_{n}$ in the exhausting sequence with $W_{k} \cup D \subset V_{n}$. By considering the passage of $V_{n}$ to $W_{n}$ we can rechoose the disc spanning $\partial D$ to obtain a new compressing disc $E \subset W_{n}$. Since $\partial W$ is incompressible in $M \backslash \operatorname{int}\left(W_{k}\right)$, it follows that $E$ must hit a component of $W_{k}$ distinct from $W$. This imples that $W_{n}$ contains fewer components than $W_{k}$, which is a contradiction.

Lemma 5.18. If $A_{1}, A_{2}, \cdots, A_{n}$ are as in Lemma 5.17, with pairwise disjoint endreductions $\mathcal{W}_{A_{1}}, \mathcal{W}_{A_{2}}, \cdots, \mathcal{W}_{A_{n}}$, then $\mathcal{W}_{A_{1}} \cup \mathcal{W}_{A_{2}} \cup \cdots \cup \mathcal{W}_{A_{n}}$ is $H_{1}$-injective in $M$, in both $\mathbb{Z}$ and $\mathbb{Z} / 2 \mathbb{Z}$ coefficients. 
Proof. Repeat the proof of Lemma 5.11.

Corollary 5.19. Let $A$ be a union of finitely many pairwise disjoint closed curves in the end-manifold $M$. If each component of $A$ is homotopically nontrivial, then $A$ breaks up into at most rank $\left(\pi_{1}(M)\right)$ maximal nonseparable subsets.

Proof. If $A$ partitions into maximal nonseparable subsets $A_{1}, \cdots, A_{n}$, then the $H_{1^{-}}$ rank of $\mathcal{W}_{A_{i}}$ is nontrivial, since $\pi_{1}\left(\mathcal{W}_{A_{i}}\right)$ is a nontrivial subgroup of a free/surface group. Now apply the previous lemma.

Lemma 5.20. Let $\gamma_{1}, \gamma_{2}, \cdots$ be a sequence of homotopically nontrivial, pairwise disjoint closed curves in the end-manifold $M$. Then we can group together finitely many of the curves into $\gamma_{1}$ and pass to a subsequence so that

(1) Any finite subset of $\left\{\gamma_{1}, \gamma_{2}, \cdots\right\}$ which contains $\gamma_{1}$ is end-nonseparable.

(2) Each component of $\gamma_{1}$, and each $\gamma_{i}, i \geq 2$ represent the same element of $H_{1}(M, \mathbb{Z} / 2 \mathbb{Z})$.

Proof. By passing to a subsequence we can assume that each $\gamma_{i}$ represents the same element of $H_{1}(M, \mathbb{Z} / 2 \mathbb{Z})$. By Lemma 5.16, if $T$ is a finite subset of $\Gamma$, then $T$ canonically partitions into finitely many end-nonseparable subsets $S_{1}, \cdots, S_{n}$ with corresponding pairwise disjoint end-reductions $\mathcal{W}_{1}, \cdots, \mathcal{W}_{n}$. Define

$$
\begin{aligned}
C(T)=\sum_{i=1}^{n} \operatorname{rank}\left(H_{1}\left(\mathcal{W}_{i}, \mathbb{Z} / 2 \mathbb{Z}\right)\right) & =\operatorname{rank}\left(H_{1}\left(\bigcup_{i=1}^{n} \mathcal{W}_{i}, \mathbb{Z} / 2 \mathbb{Z}\right)\right) \\
& \leq \operatorname{rank}\left(H_{1}(M, \mathbb{Z} / 2 \mathbb{Z})\right)
\end{aligned}
$$

where the last inequality follows from Lemma 5.18, Define

$$
C(\Gamma)=\max \{C(T) \mid T \text { is a finite subset of } \Gamma\} .
$$

Now pass to an infinite subset of $\Gamma$ with $C(\Gamma)$ minimal. By Lemma 5.18, if $T \subset$ $\Gamma$ with $C(T)=C(\Gamma)$, then adding a new element to the $T$ does not increase the number of end-nonseparable subsets in its canonical partition. Since $C(\Gamma)$ is minimal, we can enlarge $T$ by adding finitely many elements so that the enlarged $T$, which by abuse of notation we still call $T$, is end-nonseparable. Again by maximality of $C(T), T$ together with any finite subset of $\Gamma$ is still end-nonseparable. Now express $\Gamma$ as $\bigcup \gamma_{i}$ with $\gamma_{1}=T$.

Theorem 5.21 (Infinite end-engulfing theorem). If $\gamma_{1}, \gamma_{2}, \cdots$ is a locally finite sequence of pairwise disjoint, homotopically nontrivial, closed curves in the endmanifold $M$, then after passing to a subsequence, allowing $\gamma_{1}$ to have multiple components and fixing a base point $x \in \gamma_{1}$, there exist compact submanifolds $D \subset W_{1} \subset$ $W_{2} \subset \cdots$ of $M$ such that

(1) $\partial W_{i} \cap \partial M$ is a union of components of $\partial M$ and $\partial W_{i}-\partial M$ is connected.

(2) If $\Gamma_{i}=\bigcup_{j=1}^{i} \gamma_{j}$, then $\Gamma_{i} \subset W_{i}$, and $\Gamma_{i}$ can be homotoped into $D$ via a homotopy supported in $W_{i}$.

(3) $\partial W_{i}$ is 2-incompressible rel $\Gamma_{i}$.

(4) If $\mathcal{W}=\bigcup W_{i}$, then $\mathcal{W}$ is $\pi_{1}$ and $H_{1}$-injective in both $\mathbb{Z}$ and $\mathbb{Z} / 2 \mathbb{Z}$ coefficients.

(5) $D$ is a core of $\mathcal{W}$ and is of the form $\partial \mathcal{W} \times I \cup 1$-handles.

The conclusion of this theorem is schematically depicted in Figure 6 


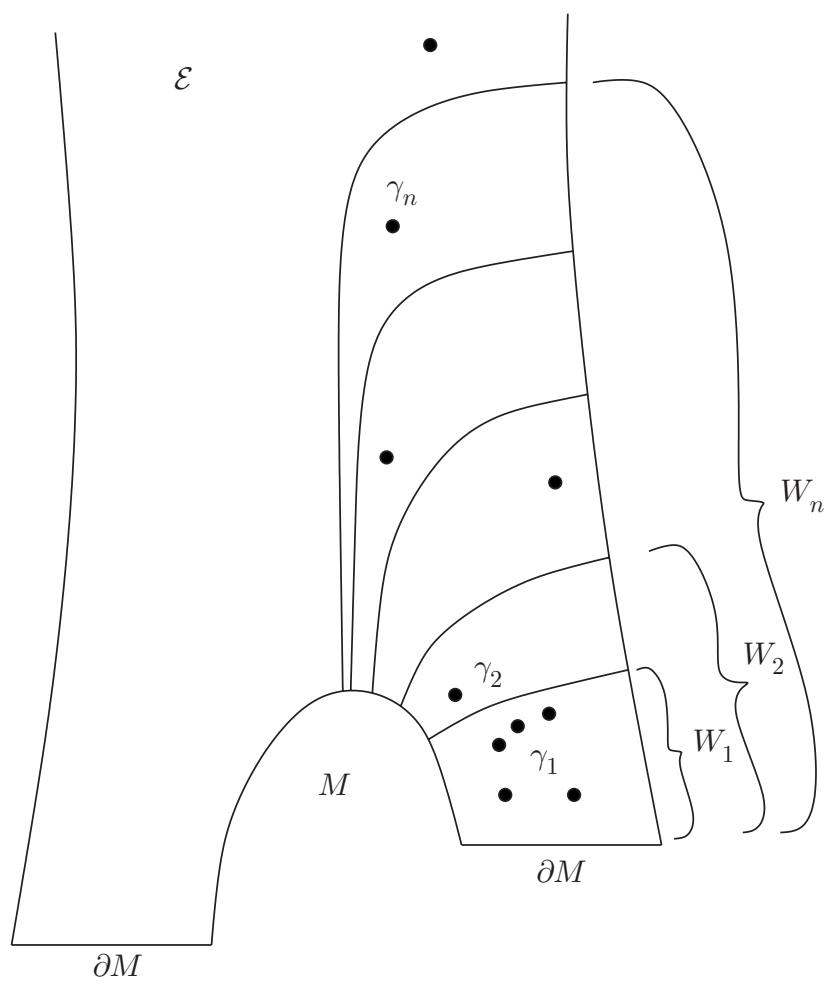

Figure 6. A schematic view of $\left\{W_{i}\right\},\left\{\gamma_{i}\right\}$ and $M$.

Proof. By passing to a subsequence and allowing $\gamma_{1}$ to have multiple components we can assume that $\Gamma=\left\{\gamma_{i}\right\}$ satisfies the conclusions of Lemma 5.20. Assume that $\gamma_{1}$ has at least two elements.

Let $\mathcal{W}_{1}$ be an end-reduction to $\gamma_{1}$ with standard exhaustion $W_{1,1} \subset W_{1,2} \subset \cdots$. Let $D_{1}$ be a core for $\mathcal{W}_{1}$ with $x \in D_{1}$. Since $\mathcal{W}_{1}$ is $\pi_{1}$-injective in $M$, by passing to a subsequence we can choose $W_{1,1}$ so that each component of $\gamma_{1}$ can be homotoped into $D$ via a homotopy in $W_{1,1}$. Furthermore, $H_{1}$-injectivity allows us to assume that within $W_{1,1}$ each $\gamma \in \gamma_{1}$ is $\mathbb{Z}_{2}$-homologous to a component $\gamma^{\prime}$ of $\gamma_{1}$ with $\gamma^{\prime} \neq \gamma$. If $\gamma$ represents the trivial class, it should be homologically trivial in $W_{1,1}$. Finally $W_{1,1}$ should be sufficiently large so that $\partial W_{1,1}$ is a union of components of $\partial M$ and a single component disjoint from $\partial M$. Note that $\partial W_{1,1}$ is 2-incompressible rel $\gamma_{1}$. Indeed, by construction $\partial W_{1,1}$ is incompressible in $M / \gamma_{1}$; hence $\partial W_{1,1}$ is incompressible to the outside and any essential compressing disc $D$ for $W_{1,1}$ must intersect $\gamma_{1}$ at least once. If $D$ meets the component $\gamma$ of $\gamma_{1}$, then since $\gamma$ is either $\mathbb{Z}_{2}$-homologically trivial in $W_{1,1}$ or $\mathbb{Z}_{2}$-homologous to a $\gamma^{\prime} \neq \gamma$ it follows that $\left|D \cap \gamma_{1}\right| \geq 2$.

By passing to a subsequence of $\left\{\gamma_{i}\right\}$ we can assume that $\gamma_{2} \cap W_{1,1}=\emptyset$. By Lemma 5.20] $\Gamma_{2}=\left\{\gamma_{1}, \gamma_{2}\right\}$ is end-nonseparable. Let $\mathcal{W}_{2}$ be an end-reduction for $\Gamma_{2}$ with standard exhaustion $W_{2,2} \subset W_{2,3} \subset W_{2,4} \subset \cdots$ where $W_{1,1} \subset \operatorname{int}\left(W_{2,2}\right)$. As above let $D_{2}$ be a core for $\mathcal{W}_{2}$ with $x \in D_{2}$ and by choosing $W_{2,2}$ sufficiently large we can assume that it supports a homotopy of $\Gamma_{2}$ into $D_{2}$ as well as homologies 
between elements of $\Gamma_{2}$. Finally, $\partial \mathcal{W}_{2,2}$ is a union of components of $\partial M$ and a single component disjoint from $\partial M$. As above, $\partial \mathcal{W}_{2,2}$ is 2-incompressible rel $\Gamma_{2}$.

Having inductively constructed $\Gamma_{i-1}, W_{i-1, i-1}$ and $D_{i-1}$, pass to a subsequence of $\left\{\gamma_{j}\right\}$ so that $\Gamma_{i-1} \subset \Gamma_{i}=\left\{\gamma_{1}, \cdots, \gamma_{i}\right\}$ with $\gamma_{i} \cap W_{i-1, i-1}=\emptyset$. Let $\mathcal{W}_{i}$ be an end-reduction of $\Gamma_{i}$ with standard exhaustion $W_{i, i} \subset W_{i, i+1} \subset W_{i, i+2} \subset \cdots$, where $W_{i-1, i-1} \subset \operatorname{int}\left(W_{i, i}\right)$. Let $D_{i}$ be a core of $\mathcal{W}_{i}$, but if possible let $D_{i}=D_{i-1}$. Finally $W_{i, i}$ should be chosen sufficiently large to support homotopies of $\Gamma_{i}$ into $D_{i}$ and homologies as described in the previous paragraphs and so that $\partial W_{i, i}$ is a union of components of $\partial M$ and a single other component. As above $\partial W_{i, i}$ is 2-incompressible rel $\Gamma_{i}$.

Let $\mathcal{W}=\bigcup_{i} W_{i, i}$. The proof of Lemma 5.11 shows that $\mathcal{W}$ is $\pi_{1}$ and $H_{1^{-}}$ injective in $M$ in $\mathbb{Z}$ and $\mathbb{Z}_{2}$ coefficients. By Lemma 5.5. $\mathcal{W}$ has a finitely generated fundamental group and hence for some $n$, the inclusion $W_{n, n} \rightarrow \mathcal{W}$ is $\pi_{1}$-surjective. Since $D_{n}$ and $W_{n, n}$ have the same $\pi_{1}$-image in $\pi_{1}\left(\mathcal{W}_{n}\right)$ and hence in $\pi_{1}(M)$, they have the same image in $\pi_{1}(\mathcal{W})$ and hence $D_{n} \rightarrow \mathcal{W}$ is $\pi_{1}$-surjective. Since the inclusions $D_{n} \rightarrow \mathcal{W}_{n} \rightarrow M$ are $\pi_{1}$-injective it follows that $D_{n}$ is also $\pi_{1}$-injective in $\mathcal{W}$ and hence is a core of $\mathcal{W}$. Therefore, if $m \geq n$, then $D_{n}$ and $W_{m, m}$ have the same $\pi_{1}$-image in $\pi_{1}(\mathcal{W})$ and hence in $\pi_{1}(M)$. Since the $\mathcal{W}_{m}$ is $\pi_{1}$-injective in $M$, it follows that $D_{m}$ and $D_{n}$ have the same image in $\pi_{1}\left(\mathcal{W}_{m}\right)$ and hence $D_{n}$ is a core of $\mathcal{W}_{m}$ for all $m \geq n$.

To complete the proof of the theorem reorganize $\left\{\gamma_{i}\right\}$ so that $\gamma_{1}$ is now the old $\left\{\gamma_{1}, \gamma_{2}, \cdots, \gamma_{n}\right\}$, and for all $i \in \mathbb{N}, \gamma_{1+i}=$ old $\gamma_{n+i}$. Let $D=D_{n}$. Finally, for $i \in \mathbb{N}$, let $W_{i}=W_{n+i-1, n+i-1}$.

By Corollary 5.6 each $\mathcal{W}_{i}$ is an end-manifold and hence $D$ could have been taken to be of the form $N(\partial \mathcal{W}) \times I \cup 1$-handles if $\partial \mathcal{W} \neq \emptyset$ and a handlebody otherwise.

Definition 5.22. Call the $\mathcal{W}$ constructed in Theorem 5.21 an end-engulfing of $\Gamma$.

The material in the rest of this chapter will not be used elsewhere in this paper; in particular, it is not used to prove any of the results of $\S 0$.

Lemma 5.23. If $J \subset J^{\prime}$ are finite, end nonseparable unions of homotopically essential, pairwise disjoint, closed curves with end-reductions $\mathcal{W}$ and $\mathcal{W}^{\prime}$, then $\mathcal{W}$ is isotopic rel $J$ to $\mathcal{W}_{1}$, where $\mathcal{W}_{1} \subset \mathcal{W}^{\prime}$.

Proof. Let $W_{1} \subset W_{2} \subset \cdots$ be a standard exhaustion of $\mathcal{W}$. Let $Z_{1} \subset Z_{2} \subset \cdots$ be a standard exhaustion of $\mathcal{W}^{\prime}$ arising from the exhaustion $\left\{V_{i}\right\}$ of $M$. By passing to a subsequence we can assume that $W_{1} \subset V_{1}$. By considering the passage of $V_{1}$ to $Z_{1}$ we can isotope $W_{1}$ rel $J$ to lie in $Z_{1}$. Proceeding by induction and passing to a subsequence, we can assume that $W_{k} \subset V_{k}$ and $W_{k-1} \subset Z_{k-1}$. By considering the passage of $V_{k}$ to $Z_{k}$ (which fixes $Z_{k-1}$ ) we can isotope $W_{k}$ rel $W_{k-1}$ to lie in $Z_{k}$. The isotoped $W_{i}$ 's give rise to an isotopy of $\mathcal{W}$ to $\mathcal{W}_{1}$ with $\mathcal{W}_{1} \subset \mathcal{W}^{\prime}$.

Remark 5.24. Given $J \subset J^{\prime}$ with end-reductions $\mathcal{W}$ and $\mathcal{W}^{\prime}$ one can isotope $\mathcal{W}$ rel $J$ to $\mathcal{W}_{1}$ so that $\mathcal{W}_{1} \subset \mathcal{W}^{\prime}$ (Lemma 5.23). On the other hand one cannot in general isotope $\mathcal{W}^{\prime}$ to contain $\mathcal{W}$. One need only look at the case of $J \subset J^{\prime}$ being nested balls in the Whitehead manifold to find examples. Such considerations make it challenging to find nested end-reductions $\mathcal{W}_{1} \subset \mathcal{W}_{2} \subset \mathcal{W}_{3} \subset \cdots$.

Theorem 5.25 (Finite end-reduction theorem). Let $M$ be an end-manifold. If $\Gamma=\left\{\gamma_{i}\right\}$ is an end-nonseparable union of finitely many homotopically essential, 
pairwise disjoint, closed curves, then an end-reduction $\mathcal{W}_{\Gamma}$ of $\Gamma$ has finitely generated fundamental group and given a standard exhaustion $\left\{W_{i}\right\}$, by passing to a subsequence, for all $i, j<k$,

$$
i n_{*}\left(\pi_{1}\left(W_{i}\right)\right)=i n_{*}\left(\pi_{1}\left(W_{j}\right)\right) \subset \pi_{1}\left(W_{k}\right)
$$

and the map $i_{*}: \pi_{1}\left(W_{k}\right) \rightarrow \pi_{1}\left(\mathcal{W}_{\Gamma}\right)$ restricted to $i n_{*}\left(\pi_{1}\left(W_{i}\right)\right)$ induces an isomorphism onto $\pi_{1}\left(\mathcal{W}_{\Gamma}\right)$. Here $i_{*}$ denotes the map induced by inclusion.

We first prove a topological lemma.

Lemma 5.26. If $M$ is an end-manifold, then $M$ has an exhaustion by compact manifolds $V_{1} \subset V_{2} \subset \cdots$, such that for each $i>1$ either $V_{i}$ is a handlebody, in which case $\partial M=\emptyset$, or $V_{i}$ is obtained by attaching 1-handles to an $N(\partial M)$.

Proof. If $\partial M=\emptyset$, then $\pi_{1}(M)$ is free and this result follows directly from [FF]. If $\partial M \neq \emptyset$, it suffices to show that if $X$ is any compact submanifold of $M$, then $X \subset V$, where $V$ is obtained by thickening $\partial M$ and attaching 1-handles. We use the standard argument; e.g., see $\overline{\mathrm{BF}}$, $\mathrm{BT} 2$ or $\mathrm{FF}$. Using the loop theorem we can pass from $X$ to a submanifold $Y$, with incompressible boundary via a sequence of compressions and external 2-handle additions. By appropriately enlarging $X$ to $X_{1}$, so as to contain these 2-handles, we can pass from $X_{1}$ to $Y$ by only compressions. By enlarging $Y$, and hence $X_{1}$, we can assume that $\partial M \subset Y$ and no component of $M \backslash \operatorname{int}(Y)$ is compact. By Lemma 5.6 each component of $\partial Y$ is boundary parallel and hence $Y$ is of the form $N(\partial M) \cup 1$-handles.

Proof of Theorem [5.25. Let $V_{1} \subset V_{2} \subset \cdots$ be an exhaustion of $M$ as in Lemma 5.26 so that $\Gamma \subset V_{1}$. Let $W_{1} \subset W_{2} \subset \cdots$ be a standard exhaustion of $\mathcal{W}_{\Gamma}$ arising from the exhaustion $\left\{V_{i}\right\}$ of $M$.

By Definition 5.14 and Lemma $5.12 \pi_{1}\left(\mathcal{W}_{\Gamma}\right)$ is finitely generated, so we can pass to a subsequence and assume that the induced map $\pi_{1}\left(W_{1}\right) \rightarrow \pi_{1}\left(\mathcal{W}_{\Gamma}\right)$ is surjective.

Let $H_{i}=\operatorname{in}_{*}\left(\pi_{1}\left(W_{1}\right)\right)$, where in : $W_{1} \rightarrow W_{i}$ is inclusion. We now show that after passing to a subsequence of the $W_{i}^{\prime} s, i \geq 2$, the induced maps

$$
H_{2} \rightarrow H_{3} \rightarrow \cdots \rightarrow \pi_{1}\left(\mathcal{W}_{\Gamma}\right)
$$

are all isomorphisms.

For $j \geq 1$, let $G_{j}=\alpha_{*}^{j}\left(\pi_{1}\left(W_{1}\right)\right)$, where $\alpha^{j}: W_{1} \rightarrow V_{j}$ is inclusion. Each $G_{j}$ is a finitely generated subgroup of $\pi_{1}\left(V_{j}\right)$ and hence is a free product of finitely many closed orientable surface groups and a finitely generated free group. Since for all $j$, $\operatorname{rank}\left(G_{j}\right) \leq \operatorname{rank}\left(G_{1}\right)$, there are only finitely many possibilities for such groups, and hence by passing to a subsequence we can assume that for $j, k>1$, the groups $G_{j}$ and $G_{k}$ are abstractly isomorphic. Free/surface groups are obviously linear, hence residually finite by Malcev $[\mathrm{Mv}$. Furthermore, Malcev $\overline{\mathrm{Mv}}$ ] went on to show that finitely generated residually finite groups are Hopfian; i.e., surjective self maps are isomorphisms. This implies that the induced maps $G_{2} \rightarrow G_{3} \rightarrow G_{4} \rightarrow \cdots$ are all isomorphisms. If

$$
K=\operatorname{ker}\left(\mathrm{in}_{*}: \pi_{1}\left(W_{1}\right) \rightarrow \pi_{1}\left(\mathcal{W}_{\Gamma}\right)\right)
$$

then

$$
K=\operatorname{ker}\left(\pi_{1}\left(W_{1}\right) \rightarrow \pi_{1}(M)\right)=\operatorname{ker}\left(\pi_{1}\left(W_{1}\right) \rightarrow G_{2}\right) .
$$

We now show that $K=\operatorname{ker}\left(\pi_{1}\left(W_{1}\right) \rightarrow \pi_{1}\left(W_{2}\right)\right)$. One readily checks that if $W_{1} \subset V$ and $K=\operatorname{ker}\left(\pi_{1}\left(W_{1}\right) \rightarrow \pi_{1}(V)\right)$, and $V^{\prime}$ is obtained from $V$ by compression, 2-handle addition or deletion, where these operations are performed in 
the complement of $W_{1}$, then $K \subset \operatorname{ker}\left(\pi_{1}\left(W_{1}\right) \rightarrow \pi_{1}\left(V^{\prime}\right)\right)$. This implies that if $K_{2}=\operatorname{ker}\left(\pi_{1}\left(W_{1}\right) \rightarrow \pi_{1}\left(W_{2}\right)\right)$, then $K \subset K_{2}$. On the other hand $K_{2} \subset K$ since $K=\operatorname{ker}\left(\pi_{1}\left(W_{1}\right) \rightarrow \pi_{1}\left(\mathcal{W}_{\Gamma}\right)\right)$. Therefore, the induced maps $H_{2} \rightarrow H_{3} \rightarrow \pi_{1}\left(\mathcal{W}_{\Gamma}\right)$ are isomorphisms.

Apply the argument of the previous paragraph to obtain a subsequence of $\left\{W_{i}\right\}$ which starts with $W_{1}$ and $W_{2}$ such that the $\pi_{1}$-image of $W_{2}$ in $W_{j}, j>2$, maps isomorphically to $\pi_{1}\left(W_{\Gamma}\right)$, via the map induced by inclusion. Continue in this manner to construct $W_{3}, W_{4}, \cdots$.

\section{Addendum to Theorem 5.21}

We can obtain the following additional property. If $i, j<k$, then

$$
\operatorname{in}_{*}\left(\pi_{1}\left(W_{i}\right)\right)=\operatorname{in}_{*}\left(\pi_{1}\left(W_{j}\right)\right) \subset \pi_{1}\left(W_{k}\right),
$$

where in denotes inclusion. The map $\operatorname{in}_{*}: \pi_{1}\left(W_{k}\right) \rightarrow \pi_{1}(\mathcal{W})$ restricted to $\operatorname{in}_{*}\left(\pi_{1}\left(W_{i}\right)\right)$ induces an isomorphism onto $\pi_{1}(\mathcal{W})$.

Proof. Apply Theorem 5.21 to produce the space $D$ as well as the sets $\left\{\gamma_{i}\right\},\left\{\Gamma_{i}\right\}$, $\left\{W_{i}\right\}$, which we now relabel as $\left\{\gamma_{i}^{\prime}\right\},\left\{\Gamma_{i}^{\prime}\right\},\left\{W_{i}^{\prime}\right\}$. Define $\gamma_{1}=\Gamma_{1}=\gamma_{1}^{\prime}, W_{1}=W_{1}^{\prime}$, $\gamma_{2}=\gamma_{2}^{\prime}$ and $\Gamma_{2}=\Gamma_{2}^{\prime}$. Let $W_{2}^{\prime}=W_{2}^{1} \subset W_{2}^{2} \subset W_{2}^{3} \subset \cdots$ be a standard exhaustion of an end-reduction $\mathcal{W}_{2}$ of $\Gamma_{2}$, which we can assume satisfies the conclusions of Theorem 5.25. Defining $W_{2}=W_{2}^{2}$, we see that the restriction of $\operatorname{in}_{*}: \pi_{1}\left(W_{2}\right) \rightarrow$ $\pi_{1}\left(\mathcal{W}_{2}\right)=\pi_{1}(D)$ to $\operatorname{in}_{*}\left(\pi_{1}\left(W_{1}\right)\right) \subset \pi_{1}\left(W_{2}\right)$ is an isomorphism. Choose $\gamma_{3}=\gamma_{i_{3}}^{\prime} \in$ $\left\{\gamma_{i}^{\prime}\right\}$ so that $\gamma_{3} \cap W_{2}=\emptyset$ and define $\Gamma_{3}=\Gamma_{2} \cup \gamma_{3}$. Let $W_{3}^{1} \subset W_{3}^{2} \subset W_{3}^{3} \subset$ $\cdots$ be a standard exhaustion of an end-reduction $\mathcal{W}_{3}$ of $\Gamma_{3}$ which satisfies the conclusions of Theorem 5.25 and has $W_{2} \subset W_{3}^{1}$. Defining $W_{3}=W_{3}^{2}$, we see that the restriction of $\operatorname{in}_{*}: \pi_{1}\left(W_{3}\right) \rightarrow \pi_{1}\left(\mathcal{W}_{3}\right)=\pi_{1}(D)$ to $\operatorname{in}_{*}\left(\pi_{1}\left(W_{2}\right)\right) \subset \pi_{1}\left(W_{3}\right)$ is an isomorphism. Now define $\gamma_{3}=\gamma_{i_{3}}^{\prime}$ and $\Gamma_{2}=\Gamma_{1} \cup \gamma_{i_{3}}$. In a similar manner construct $\gamma_{4}, \gamma_{5} \cdots, \Gamma_{4}, \Gamma_{5}, \cdots, W_{4}, W_{5}, \cdots$ and finally define $\mathcal{W}=\bigcup W_{i}$.

Remarks 5.27. If one allows each $\gamma_{i}$ to be a finite set of elements, then we can obtain the conclusion (in Theorem 5.21 and its addendum) that each $\gamma_{i}$ is $\mathbb{Z} / 2 \mathbb{Z}$ homologically trivial.

Question 5.28. Let $M$ be a connected, compact, orientable, irreducible 3-manifold such that $\chi(M) \neq 0$ and let $G$ be a subgroup of $\pi_{1}(M)$. If the induced map $G /[G, G] \rightarrow H_{1}(M)$ is injective, is $G$ finitely generated?

Question 5.29. Let $\Gamma$ be a locally finite collection of pairwise disjoint homotopically essential closed curves such that $C(\Gamma)=C\left(\Gamma^{\prime}\right)$ for any infinite subset $\Gamma^{\prime}$ of $\Gamma$. Is it true, that given $n \in \mathbb{N}$, there exists an end-engulfing of $\mathcal{W}=\bigcup W_{i}$ of $\Gamma$ such that for all $i,\left|E \cap \Gamma_{i}\right| \geq n$ for all essential compressing discs $E$ of $W_{i}$ ?

\section{Proof of Theorems 0.9, 0.4 and 0.2: Parabolic free case}

Proof of Theorem 0.9 , By Lemma 5.1 and Remark 5.2 it suffices to consider the case that $\mathcal{E}$ is the end of an end-manifold $M \subset N$ such that the inclusion $M \rightarrow N$ is a homotopy equivalence. By Lemma 3.2 there exists an $\eta$-separated collection $\Delta=\left\{\delta_{i}\right\}$ of closed geodesics which exit $\mathcal{E}$. We let $\Delta_{i}$ denote the union $\Delta_{i}=\bigcup_{j \leq i} \delta_{j}$. Apply Theorem 5.21 to $\Delta$ and $M$ to pass to a subsequence, also called $\Delta$, where we allow $\delta_{1}$ to have finitely many components. Theorem 5.21 also produces a manifold 
$\mathcal{W}$ open in $M$ and exhausted by compact manifolds $\left\{W_{i}\right\}$ having the following properties.

(1) $\mathcal{W}$ is $\pi_{1}$ and $H_{1}$-injective (in $\mathbb{Z}$ and $\mathbb{Z} / 2 \mathbb{Z}$ coefficients) in $M$ and hence $\pi_{1}(\mathcal{W})$ is a free/surface group.

(2) $\partial W_{i} \backslash \partial M$ is a closed connected surface which separates $\Delta_{i}$ from $\mathcal{E}$ and is 2-incompressible in $N$ rel. $\Delta_{i}$.

(3) There exists a compact submanifold core $D \subset W_{1}$ of $\mathcal{W}$ such that for each $i, \delta_{i}$ can be homotoped into $D$ via a homotopy supported in $W_{i}$. $D$ is either of the form $\partial \mathcal{W} \times I$ with 1-handles attached to the 1 -side, if $\partial \mathcal{W} \neq \emptyset$ or a handlebody, otherwise.

Let $G_{i}$ denote $\operatorname{in}_{*}\left(\pi_{1}(D)\right) \subset \pi_{1}\left(W_{i}\right)$. Let $\partial_{e} D$ denote $D \cap \partial M=\partial \mathcal{W}$. Let $X_{i}$ denote the covering space of $W_{i}$ with group $G_{i}$ and let $\hat{D}$ denote the lift of $D$. Pick a homotopy of $\Delta_{i}$ into $D$ supported in $W_{i}$. This homotopy lifts to a homotopy of $\hat{\Delta}_{i}$ into $\hat{D}$, thereby picking out the closed preimages $\hat{\Delta}_{i}$ of $\Delta_{i}$ which are in 1-1 correspondence with $\Delta_{i}$. Let $\left\{\hat{\delta}_{1}, \cdots, \hat{\delta}_{i}\right\}$ denote these elements.

Claim. Each $W_{i}$ is a compact atoroidal Haken manifold and $\partial W_{i}$ contains a surface of genus $\geq 2$.

Proof of Claim. Each embedded torus in $N$ is compressible, since $N$ is parabolic free. A compressible torus in an irreducible 3 -manifold is either a tube, i.e. bounds a solid torus, or a convolutube, i.e. bounds a cube with knotted hole $X$, which is a 3-ball with an open regular neighborhood of a properly embedded arc removed. Furthermore, $X$ lies in a 3-ball. Therefore, if some component of $\partial W_{i}$ is a torus, $W_{i}$ is either a solid torus or a cube with knotted hole. The former can contain at most one closed geodesic and the latter none. Since $W_{i}$ contains at least two closed geodesics $\partial W_{i}$ cannot contain a torus.

If $W_{i}$ contained an embedded incompressible torus $T$, then the compact region bounded by $T$ would lie in $W_{i}$. This implies that $T$ is a convolutube. In $\mathbb{H}^{3}$, the universal covering of $N$, let $\tilde{\Delta}_{i}$ denote the preimage of $\Delta_{i}$ and $\tilde{W}_{i}$ the preimage of $W_{i}$. Since $T$ lies in a 3 -cell in $N, T$ lifts to a torus $\tilde{T}$ isometric to $T$. Using the loop theorem, it follows that $\tilde{\partial} W_{i}$ is incompressible in $\mathbb{H}^{3} \backslash \tilde{\Delta}_{i}$ and $\tilde{T}$ is incompressible in $\tilde{W}_{i}$. We will show that after an isotopy of $\tilde{T}$ supported in $\tilde{W}_{i}$, there exists an embedded 3-ball $F \subset \mathbb{H}^{3}$ such that $T \subset F$ and $F \cap \tilde{\Delta}_{i}=\emptyset$. This implies that $T$ is compressible in $F$, via a compressing disc $D$ disjoint from $\tilde{\Delta}_{i}$. Since $\tilde{W}_{i}$ is incompressible in $\mathbb{H}^{3} \backslash \tilde{\Delta}_{i}$ it follows that $D$ can be isotoped rel $\partial D$ so that $D \subset \tilde{W}_{i}$. This contradicts the fact that $\tilde{T}$ is incompressible in $\tilde{W}_{i}$.

Here is how to find $F$. Let $E \subset \mathbb{H}^{3}$ be a large round ball transverse to $\tilde{\Delta}_{i}$ which contains $\tilde{T}$ in its interior. $\tilde{\Delta}_{i} \cap E=\left\{\alpha_{1}, \cdots, \alpha_{n}\right\}$ is a finite union of unknotted arc; i.e., there exist pairwise disjoint embedded discs $\left\{D_{1}, \cdots, D_{n}\right\}$ such that for each $k, D_{k} \subset E$ and $\partial D_{k}$ consists of $\alpha_{k}$ together with an arc lying in $\partial E$. For each $k$, either $\alpha_{k} \cap \tilde{W}_{i}=\emptyset$ or $\alpha_{k} \subset \tilde{W}_{i}$. Since $\partial \tilde{W}_{i}$ is incompressible in $\mathbb{H}^{3} \backslash \tilde{\Delta}_{i}, E$ and the $D_{k}$ 's can be isotoped, via an isotopy which fixes $\tilde{\Delta}_{i}$ pointwise, to $E^{\prime}$ and $D_{k}^{\prime}$ 's so that $E^{\prime}$ is a 3-ball containing $\tilde{T}$, the $D_{k}^{\prime}$ 's are unknotting discs for the $\alpha_{k}$ 's and for each $k$, either $D_{k}^{\prime} \cap \tilde{W}_{i}=\emptyset$ or $D_{k}^{\prime} \subset \tilde{W}_{i}$. After an isotopy of $\tilde{T}$ supported in 
$E^{\prime} \cap \tilde{W}_{i}$, for each $k, \tilde{T} \cap D_{k}^{\prime}=\emptyset$. Finally let $F$ equal $E^{\prime}-\bigcup_{j=1}^{n} \operatorname{int}\left(N\left(D_{k}^{\prime}\right)\right)$, where $N\left(D_{k}^{\prime}\right)$ is a very small regular neighborhood of $D_{k}^{\prime}$.

It now follows from Thurston (see Proposition 3.2 in [Ca] or [Mo] that $\operatorname{int}\left(X_{i}\right)$ is topologically tame. Let $\bar{X}_{i}$ denote its manifold compactification. Since $\partial_{e} D$ is a union of components of $\partial W_{i}$ and $X_{i}$ is the $\pi_{1}(D)$ cover, there is a canonical identification of $\partial_{e} D$ with some set of components of $\partial \bar{X}_{i}$. Let $\partial_{e} \hat{D}$ denote these components. Having the same homotopy type as $D$, it follows by the usual grouptheoretic reasons that $\bar{X}_{i}$ either compresses down to a 3 -ball, or to a possibly disconnected (closed orientable surface) $\times I$. In the former case $\bar{X}_{i}$ is a handlebody which, for reasons of Euler characteristic, is of the same genus as $D$. In the latter case, since $\partial_{e} \hat{D}$ is an incompressible surface, $\bar{X}_{i}$ is topologically $\partial_{e} \hat{D} \times I$ with 1 handles attached to the 1 -side. Let $\bar{S}_{i}$ denote $\partial \bar{X}_{i}-\partial_{e} \hat{D}$. Again by reason of the Euler characteristic, genus $\left(\bar{S}_{i}\right)=\operatorname{genus}\left(\partial_{\mathcal{E}} D\right)$, where $\partial_{\mathcal{E}} D=\partial D-\partial_{e} D$.

Define $g^{\prime}=\operatorname{genus}\left(\bar{S}_{i}\right)=\operatorname{genus}\left(\partial_{\mathcal{E}} D\right)$. We show that $g^{\prime} \leq g=\operatorname{genus}\left(\partial_{\mathcal{E}} C\right)$, where $C$ is the original core of $M$. By construction $D \cap \partial M$ is a union of components of $C \cap \partial M$; therefore it suffices to show that the number of 1-handles attached to $N(D \cap \partial M)$ is not more than the number of 1-handles attached to $N(C \cap \partial M)$ in the constructions of $D$ and $C$ respectively. If $D \cap \partial M=C \cap \partial M$, then this follows immediately from the fact that $D$ and $C$ are cores respectively of $\mathcal{W}$ and $M$ and the $H_{1}$-injectivity of $\mathcal{W}$ in $M$. Let $E=(C \cap \partial M) \backslash(D \cap \partial M)$. The $H_{1^{-}}$ injectivity of $C$ in $M$ implies that the inclusion $H_{1}(C \cap \partial M) \rightarrow H_{1}(M)$ is injective. The $H_{1}$-injectivity of $D$ in $\mathcal{W}$ and the $H_{1}$-injectivity of $\mathcal{W}$ in $M$ implies that $D$ is $H_{1}$-injective in $M$. If the kernel of $\operatorname{in}_{*}: H_{1}(D \cup E) \rightarrow H_{1}(M)$ is nontrivial, then a nontrivial homology between $D$ and $E$ would lie in some $V_{j}$, where $j>1$ and $V_{j}$ is a term in the exhausting sequence of $M$ used for constructing $\left\{W_{i}\right\}$. Arguing as in the proof of Lemma 5.11, $W_{j}$ contains a nontrivial homology between $D$ and $E$ and hence, $E \cap W_{j} \neq \emptyset$. This contradicts the fact that $W_{j} \cap \partial M=D \cap \partial M$. Therefore

(*) number of 1-handles of $D \leq \operatorname{rank} H_{1}(M)-\left(\operatorname{rank} H_{1}(D \cap \partial M)+\operatorname{rank} H_{1}(E)\right)$

$$
\begin{aligned}
& =\operatorname{rank} H_{1}(M)-\operatorname{rank}\left(H_{1}(\partial C \cap M)\right) \\
& =\text { number of 1-handles of } C .
\end{aligned}
$$

Isotope $\bar{S}_{i} \subset \bar{X}_{i}$ to an embedded surface $\hat{S}^{i} \subset X_{i}$ via an isotopy which does not cross $\hat{\Delta}_{i}$. Next, if possible, compress $\hat{S}^{i}$ via a compression either disjoint from $\hat{\Delta}_{i}$ or crossing $\hat{\Delta}_{i}$ once, say at $\hat{\delta}_{i_{1}}$. If possible, compress the resulting surface via a compression crossing $\hat{\Delta}_{i} \backslash \hat{\delta}_{i_{1}}$ at most once and so on. Since genus $\left(\hat{S}^{i}\right)=$ $g^{\prime}$, there is an a priori upper bound on the number of compressions we need to do. In the end we obtain connected surfaces $\hat{S}_{1}^{i}, \cdots, \hat{S}_{n}^{i}$ in $X_{i}$ which are 2incompressible rel $\hat{\Delta} \backslash\left\{\hat{\delta}_{i_{1}}, \cdots, \hat{\delta}_{i_{m}}\right\}$ where $m<2 g^{\prime}-1$, and both $n$ and $\operatorname{genus}\left(\hat{S}_{j}^{i}\right)$ are $\leq g^{\prime}$. Since $X_{i} \backslash \hat{\Delta}_{i}$ is irreducible, we can assume that no $\hat{S}_{j}^{i}$ is a 2 -sphere. These $\hat{S}_{j}^{i}$ 's create a partition $B_{1}^{i}, \cdots B_{n}^{i}$ of $\hat{\Delta}_{i} \backslash\left\{\hat{\delta}_{i_{1}}, \cdots, \hat{\delta}_{i_{m}}\right\}$, where $B_{j}^{i}$ is the subset of $\hat{\Delta}_{i} \backslash\left\{\hat{\delta}_{i_{1}}, \cdots, \hat{\delta}_{i_{m}}\right\}$ separated from $\bar{S}_{i}$ by $\hat{S}_{j}^{i}$. Each $\hat{S}_{j}^{i}$ is incompressible to the $\bar{S}_{i}$ side, since the component of $\bar{X}_{i}$ split along $\hat{S}_{1}^{i} \cup \cdots \cup \hat{S}_{n}^{i}$ which contains $\bar{S}_{i}$ is homeomorphic to $N\left(\hat{S}_{1}^{i} \cup \cdots \cup \hat{S}_{n}^{i}\right) \cup 1$-handles. Therefore, each $\hat{S}_{j}^{i}$ is 2-incompressible rel $B_{j}^{i}$.

As in the proof of Canary's theorem, after appropriately reordering the $B_{j}^{i}$ 's we can find a $p \in \mathbb{N}$ and a sequence $k_{1}<k_{2}<\cdots$ such that $\hat{\delta}_{p} \subset B_{1}^{k_{i}}$ and if $p(i)$ 
denotes the largest index of a $\hat{\delta}_{j} \in B_{1}^{k_{i}}$, then $\lim _{i \rightarrow \infty} p(i)=\infty$. In general reorder the $\hat{S}_{j}^{i}$ 's so that, if possible, $\hat{\delta}_{p} \subset B_{1}^{i}$.

Fix $i$. Let $W_{i}^{\prime}$ be the union of $W_{i}$ together with the components of $N \backslash \operatorname{int}(M)$ which nontrivially intersect $\partial W_{1}$. Let $Y_{i}$ denote the covering of $W_{i}^{\prime}$ with fundamental group $G_{i}$. View $X_{i}, \hat{\Delta}_{i}$, and the $\hat{S}_{j}^{i}$ 's etc. as sitting naturally in $Y_{i}$. Let $\delta \in \Delta$ be disjoint from $W_{i}$. Apply Lemma 2.3 to $W_{i}, \delta \cup \Delta_{i}$ and $S_{1}^{i}$.

We have the following dictionary between terms appearing in our current setup (on the left) and the terms appearing in the hypothesis of Lemma 2.3 (on the right):

$$
\begin{aligned}
\text { the geodesics } \delta \cup \Delta_{i} & \longleftrightarrow \text { the geodesics } \Delta_{1} \\
\text { the manifold } W_{i}^{\prime} & \longleftrightarrow \text { the manifold } W \\
W_{i}^{\prime} \cap\left(\delta \cup \Delta_{i}\right)=\Delta_{i} & \longleftrightarrow W \cap \Delta_{1}=\Delta
\end{aligned}
$$

the subgroup $G_{i}$ of $\pi_{1}\left(W_{i}^{\prime}\right) \longleftrightarrow$ the subgroup $G$ of $\pi_{1}(W)$

the cover $Y_{i}$ with $\pi_{1}\left(Y_{i}\right)=G_{i} \longleftrightarrow$ the cover $X$ with $\pi_{1}(X)=G$

the lifted geodesics $B_{1}^{i} \longleftrightarrow$ the lifted geodesics $B$

the surface $\hat{S}_{1}^{i} \longleftrightarrow$ the surface $S$.

Then Lemma 2.3 constructs surfaces $T^{i}$ and $P^{i}$ where the correspondence is

the shrinkwrapped surface $T^{i} \longleftrightarrow$ the shrinkwrapped surface $T$

the approximating surface $P^{i} \longleftrightarrow$ the approximating surface $T_{t}$.

In more detail: $W_{i}^{\prime}$ is isotopic to a manifold $W_{i}^{\text {new }}$, via an isotopy fixing $\Delta_{i} \cup \delta$ pointwise. This isotopy induces a homotopy of the covering projection $\pi: Y_{i} \rightarrow$ $W_{i}^{\prime} \subset N$ to a covering projection $\pi^{\text {new }}: Y_{i} \rightarrow W_{i}^{\text {new }} \subset N$. Our $\hat{S}_{1}^{i}$ is isotopic to a surface $\hat{P}^{i}$ via an isotopy avoiding $B_{1}^{i}$, and the projection of $\hat{P}^{i}$ into $N$ is a surface $P^{i}$ which is homotopic to a CAT $(-1)$ surface $T^{i}$. Furthermore, $P^{i}$ and $T^{i}$ are at Hausdorff distance $\leq 1$ and the homotopy from $P^{i}$ to $T^{i}$ is supported within the 1-neighborhood of $P^{i}$.

We relabel superscripts, and by abuse of notation we let the sequence $\left\{T^{i}\right\}$ stand for the old subsequence $\left\{T^{k_{i}}\right\}$, with $\delta_{p\left(k_{i}\right)}$ being denoted by $\delta_{p(i)}$, etc. We also drop the superscript new so that in particular the projection $\pi: Y_{i} \rightarrow W_{i}^{\prime}$ now refers to $\pi^{\text {new }}: Y_{i} \rightarrow W_{i}^{\text {new }}$.

We use the $\delta_{p(i)}$ 's to show that $\left\{T^{i}\right\}$ exits $\mathcal{E}$. Let $\left\{\alpha_{i}\right\}$ be a locally finite collection of properly embedded rays from $\left\{\delta_{i}\right\}$ to $\mathcal{E}$. For each $i, \hat{S}_{1}^{i}$ intersects some component $\omega$ of $\pi^{-1}\left(\alpha_{p(i)}\right)$ with algebraic intersection number 1 , so $\hat{P}^{i} \cap \pi^{-1}\left(\alpha_{p(i)}\right) \neq \emptyset$. Therefore for all $i$, we have an inequality $\operatorname{dist}\left(T^{i}, \alpha_{p(i)}\right) \leq 1$. Our assertion now follows from the Bounded Diameter Lemma.

Lemma 6.1. Let $\mathcal{E}$ be an end of $M$, an orientable, irreducible 3-manifold with finitely generated fundamental group. If $C$ is a 3-manifold compact core of $M$ and $Z$ is the component of $M \backslash C$ which contains $\mathcal{E}$, then $\left[\partial_{\mathcal{E}} C\right]$ generates $H_{2}(Z)$ and is Thurston norm minimizing. Here $\partial_{\mathcal{E}} C$ is the component of $\partial C$ which faces $\mathcal{E}$.

Proof. First, $\partial_{\mathcal{E}} C$ is connected, or else there exists a closed curve $\kappa$ in $M$ intersecting a component of $\partial_{\mathcal{E}} C$ once; hence $\kappa$ is not homologous to a cycle in $C$, contradicting 


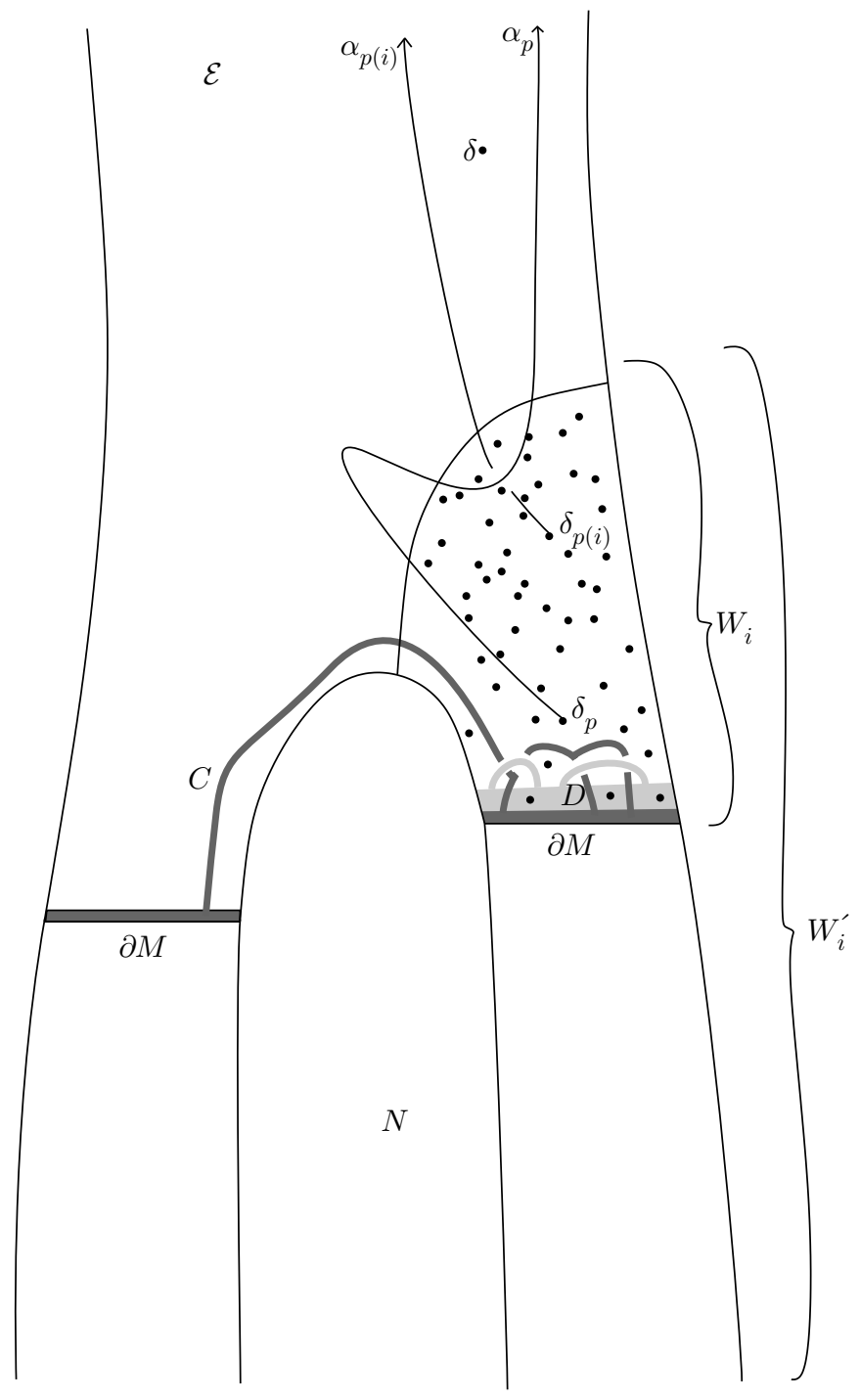

FiguRE 7.

the fact that $C$ is a core. That $\left[\partial_{\mathcal{E}} C\right]$ generates $H_{2}(Z)$ follows from the fact that any 2-cycle $w$ in $Z$ is homologous to one in $C$, so the restriction of that homology to $Z$ gives a homology of $w$ to $n\left[\partial_{\mathcal{E}} C\right]$ for some $n$. Equivalently, observe that the inclusion $C \rightarrow M$ is a homotopy equivalence, and use excision for homology.

Let $Q \subset \operatorname{int}(Z)$ be a Thurston norm-minimizing surface representing $\left[\partial_{\mathcal{E}} C\right]$. We can choose $Q$ to be connected since $H_{2}(Z)=\mathbb{Z}$. Let $V \subset Z$ be the submanifold between $\partial_{\mathcal{E}} C$ and $Q$. If genus $\left(\partial_{\mathcal{E}} C\right)>\operatorname{genus}(Q)$, then there exists a nonzero $z$ in the kernel of $\operatorname{in}_{*}: H_{1}\left(\partial_{\mathcal{E}} C\right) \rightarrow H_{1}(V)$. This follows from the well-known fact that for any compact orientable 3-manifold $V$, the rank of the kernel of the map $\operatorname{in}_{*}: H_{1}(\partial V) \rightarrow H_{1}(V)$ is $\frac{1}{2} \operatorname{rank}\left(H_{1}(\partial V)\right)$. Since $C$ is a core, $z$ is in the kernel of 
the map $\operatorname{in}_{*}: H_{1}\left(\partial_{\mathcal{E}} C\right) \rightarrow H_{1}(C)$. This gives rise to a class $w^{\prime} \in H_{2}(M)$ and dual class $z^{\prime} \in H_{1}\left(\partial_{\mathcal{E}} C\right)$ with $\left\langle z^{\prime}, w^{\prime}\right\rangle \neq 0$, which is again a contradiction.

Using this lemma, we now complete the proof of Theorem 0.9 .

Let $Z$ denote the component of $N$ split open along $\partial_{\mathcal{E}} C$ which contains $\mathcal{E}$. By Lemma 6.1, $\partial_{\mathcal{E}} C$ generates $H_{2}(Z)$. Next, observe that if $\beta$ is any ray in $Z$ from $\partial_{\mathcal{E}} C$ to $\mathcal{E}$ and $R$ is any immersed closed orientable surface in $Z$, then $[R]=n\left[\partial_{\mathcal{E}} C\right] \in$ $H_{2}(Z)$ where $\langle R, \beta\rangle=n$. To see this, note that $\left\langle n\left[\partial_{\mathcal{E}} C\right], \beta\right\rangle=n$ by considering $n$ copies of $\partial_{\mathcal{E}} C$ slightly pushed into $Z$ and whose algebraic intersection number is independent of the representative of the homology class.

We now use $\delta_{p}$ to show that for $i$ sufficiently large $\left[T^{i}\right]$ is homologous in $Z$ to $\left[\partial_{\mathcal{E}} C\right] \in H_{2}(Z) \cong \mathbb{Z}$; see Figure 7 . Let $\beta$ be the ray $\sigma * \alpha_{p}$ where $\sigma \subset Z$ is a path from $\partial_{\mathcal{E}} C$ to $\partial \alpha_{p}$. In what follows assume that $i$ is sufficiently large so that

$$
N_{2}\left(P^{i}\right) \cap\left(\sigma \cup \delta_{p} \cup C\right)=\emptyset,
$$

where $N_{2}\left(P^{i}\right)$ denotes the 2-neighborhood of $P^{i}$, and hence

$$
\left\langle T^{i}, \beta\right\rangle=\left\langle P^{i}, \alpha_{p}\right\rangle .
$$

We now compute this value. By perturbing $P^{i}$, if necessary, we can assume that $P^{i}$ is transverse to $\alpha_{p}$ and no intersections occur at double points of $P^{i}$. There is a $1-1$ correspondence of sets

$$
\left\{\alpha_{p} \cap P^{i}\right\} \longleftrightarrow\left\{\pi^{-1}\left(\alpha_{p}\right) \cap \hat{P}^{i}\right\} .
$$

Let $\mathrm{Bag}_{i}$ denote the component of $Y_{i}$ split along $\hat{P}^{i}$ which is disjoint from $\bar{S}_{i}$. Note that $\pi^{-1}\left(\delta_{p}\right) \cap \partial \operatorname{Bag}_{i}=\emptyset$. If $\kappa$ is a component of $\pi^{-1}\left(\alpha_{p}\right)$, then $\left\langle\kappa, \hat{P}^{i}\right\rangle=0$ if no endpoints lie in $\operatorname{Bag}_{i}$ while $\left\langle\kappa, \hat{P}^{i}\right\rangle=1$ if exactly one endpoint lies in $\operatorname{Bag}_{i}$. To see this, orient $\alpha_{p}$ so that the positive end escapes to $\mathcal{E}$. Then the positive end of each lift $\kappa$ is in $\partial Y_{i}$, which is outside $\mathrm{Bag}_{i}$. It follows that if $p$ is an endpoint of $\kappa$ in $\operatorname{Bag}_{i}$, then $p$ is the negative end of $\kappa$, and $\left\langle\kappa, \hat{P}^{i}\right\rangle=1$. Since $\hat{\delta}_{p}$ lies in $\operatorname{Bag}_{i}$, there is at least 1 component $\kappa$ of $\pi^{-1}\left(\alpha_{p}\right)$ with such an endpoint in $\mathrm{Bag}_{i}$, and therefore

$$
\left\langle\pi^{-1}\left(\alpha_{p}\right), \hat{P}^{i}\right\rangle \geq 1
$$

and hence

$$
\left[T^{i}\right]=n\left[\partial_{\mathcal{E}} C\right] \in H_{2}(Z)
$$

for some $n \geq 1$.

Therefore

$$
\left|\chi\left(\partial_{\mathcal{E}} C\right)\right| \geq\left|\chi\left(T_{i}\right)\right| \geq x_{s}\left(n\left[\partial_{\mathcal{E}} C\right]\right)=x\left(n\left[\partial_{\mathcal{E}} C\right]\right)=n x\left(\left[\partial_{\mathcal{E}} C\right]\right)=n\left|\chi\left(\partial_{\mathcal{E}} C\right)\right|
$$

and hence $n=1$ and $\operatorname{genus}\left(T^{i}\right)=\operatorname{genus}\left(\partial_{\mathcal{E}} C\right.$ ). Here $x$ (resp. $\left.x_{s}\right)$ denotes the Thurston (resp. singular Thurston) norm on $H_{2}(Z)$. The first inequality follows by construction, the second by definition, the third since $x_{s}=x$ (G1]), the fourth since $x$ is linear on rays $\mathrm{T2}$, and the fifth by Lemma 6.1. This completes the proof of Theorem 0.9

Remark 6.2. Since for $i$ sufficiently large, genus $\left(T^{i}\right)=g$, it follows that for such $i$, no compressions occur in the passage from $\bar{S}^{i}$ to $\hat{S}_{1}^{i}$. This mirrors the similar phenomenon seen in the proof of Canary's theorem. If the shrinkwrapped $\partial W_{i}^{\prime}$ is actually a $\Delta_{i}$-minimal surface disjoint from $\Delta_{i}$, then $\partial X_{i}$ is a least area minimal surface for the hyperbolic metric, and we can pass directly from $\hat{S}_{1}^{i}$ to a $\hat{\Delta}_{i}$-minimal surface $\hat{T}^{i}$ by shrinkwrapping in $X_{i}$. Our $T^{i}$ is then the projection of $\hat{T}^{i}$ to $N$. 
If the shrinkwrapped $\partial W_{i}^{\prime}$ touches $\Delta_{i}$, then we can still shrinkwrap $\hat{S}_{1}^{i}$ in $X_{i}$. In this case $X_{i}$ is bent and possibly squeezed along parts of $\hat{\Delta}_{i}$ and it is cumbersome to discuss the geometry and topology of $X_{i}$. Therefore we choose for the purposes of exposition to express $T^{i}$ as a limit of surfaces. These surfaces are projections of $g_{t_{k}}$-minimal surfaces in the smooth Riemannian manifolds $X_{i}$ with Riemannian metrics $g_{t_{k}}$. As metric spaces, the $\left(X_{i}, g_{t_{k}}\right)$ converge to the bent and squeezed hyperbolic "metric" on $X_{i}$.

Tameness Criteria. Let $\mathcal{E}$ be an end of the complete hyperbolic 3-manifold $N$ with finitely generated fundamental group and compact core $C$. Let $Z$ be the component of $N \backslash \operatorname{int}(C)$ containing $\mathcal{E}$ with $\partial_{\mathcal{E}} C$ denoting $\partial Z$. Let $T_{1}, T_{2}, \cdots$ be a sequence of surfaces mapped into $N$. Consider the following properties.

(1) $\operatorname{genus}\left(T_{i}\right)=\operatorname{genus}\left(\partial_{\mathcal{E}} C\right)$.

(2) $T_{i} \subset Z$ and exit $\mathcal{E}$.

(3) Each $T_{i}$ homologically separates $C$ from $\mathcal{E}$ (i.e., $\left[T_{i}\right]=\left[\partial_{\mathcal{E}} C\right] \in H_{2}(Z)$ ).

(4) Each $T_{i}$ is $\operatorname{CAT}(-1)$.

Theorem 6.3 (Souto [So]). If $T_{1}, T_{2}, \cdots$ is a sequence of mapped surfaces in the complete hyperbolic 3-manifold $N$ with core $C$ and end $\mathcal{E}$ which satisfies Criteria (1), (2) and (3), then $\mathcal{E}$ is topologically tame.

Theorem 6.3 follows directly from the proof of Theorem 2, $[\mathrm{So}$. That proof makes essential use of the work of Bonahon [Bo] and Canary [Ca]. We now show how Criterion (4) enables us to establish tameness without invoking the impressive technology of [Bo] and [Ca]. Our argument, inspired in part by Souto's work, requires only elementary hyperbolic geometry and basic 3-manifold topology.

A topological argument that criteria (1)-(4) imply tameness. It suffices to consider the case that $\mathcal{E}$ is the end of an end-manifold $M$ which includes by a homotopy equivalence into $N$, and that $C \subset M$ is of the form $\partial M \times I \cup 1$-handles, where the 1-handles attach to $\partial M \times 1$ and $\partial M=\partial M \times 0$.

Using standard arguments, we can replace the $T_{i}$ 's by simplicial hyperbolic surfaces as defined in [Ca]. The idea of how to do this is simple: the CAT $(-1)$ property implies that each $T_{i}$ has an essential simple closed curve $\kappa_{i}$ of length uniformly bounded above. If $\kappa_{i}^{*}$ denotes the geodesic in $N$ homotopic to $\kappa_{i}$, then either the $\kappa_{i}^{*}$ have length bounded below by some constant, and are therefore contained within a bounded neighborhood of $\kappa_{i}$, or else the lengths of the $\kappa_{i}^{*}$ get arbitrarily short, and therefore they escape to infinity. In either case, the sequence $\kappa_{1}^{*}, \kappa_{2}^{*}, \cdots$ exits $\mathcal{E}$. Then we can triangulate $T_{i}$ by a 1 -vertex triangulation with a vertex on $\kappa_{i}^{*}$ and pull the simplices tight to geodesic triangles. This produces a simplicial hyperbolic surface, homotopic to $T_{i}$, which is contained in a bounded neighborhood of $\kappa_{i}^{*}$ rel. the thin part of $N$, and therefore these surfaces also exit $\mathcal{E}$. From now on we assume that each $T_{i}$ is a simplicial hyperbolic surface.

Note that either $\partial_{\mathcal{E}} C$ is incompressible in $N$ and hence $M$ is homotopy equivalent to $\partial_{\mathcal{E}} C \times[0, \infty)$ or each $T_{i}$ is compressible in $N$; i.e., there exists an essential simple closed curve in $T_{i}$ that is homotopically trivial in $N$. Indeed, using the $\pi_{1}$-surjectivity of $C$ and the irreducibility of $N, T_{i}$ can be homotoped into $C$. If $T_{i}$ is incompressible in $N$, then $T_{i}$ can be homotoped off the 1-handles and then homotoped into a component of $\partial M$. Using Criterion (3), the degree of this map 
is one, which implies that $T_{i}$ is homotopic to a homeomorphism onto a component of $\partial M$. Since genus $\left(T_{i}\right)=\operatorname{genus}\left(\partial_{\mathcal{E}} C\right)$, it follows that $C=\partial M \times I$ and hence $\partial_{\mathcal{E}} C$ is incompressible in $N$.

Since either $M$ is homotopy equivalent to $\partial M \times[0, \infty)$ or each $T_{i}$ is compressible, it follows by Canary [Ca] and Canary-Minsky $[\mathrm{CaM}]$ (see also Proposition 3 in $[\underline{\mathrm{So}}$ ) that there exists a compact set $K \subset Z$ such that each $T_{i}$ can be homotoped within $Z$ to a simplicial hyperbolic surface $T_{i}^{0}$ which nontrivially intersects $K$. Here $Z$ is the closure of $M-\operatorname{int}(C)$. By the Bounded Diameter Lemma, there exists a compact set $K_{1} \subset Z$ such that for each $i, T_{i}^{0} \subset K_{1}$.

Since $x=x_{s}$ G1, there exists a sequence of embedded genus- $g$ surfaces $A_{1}, A_{2}$, *. such that for each $i, A_{i}$ lies in a small neighborhood of $T_{i}$ and $\left[A_{i}\right]=\left[T_{i}\right]=$ $\left[\partial_{\mathcal{E}} C\right] \in H_{2}(Z)$. By passing to subsequence we can assume that the $A_{i}$ 's are pairwise disjoint and each $A_{i}$ is disjoint from $K_{1}$ and separates $\mathcal{E}$ from $K_{1}$. Let $A_{[p, q]}$ denote the compact region between $A_{p}$ and $A_{q}$. Since genus $\left(A_{p}\right)=\operatorname{genus}\left(\partial_{\mathcal{E}} C\right)$, it follows by Lemma 6.1 that $A_{p}$ is Thurston norm-minimizing in $Z$ and hence is $\pi_{1}$-injective in $Z$ and in $A_{[p, p+1]}$.

To establish tameness it suffices to show that each $A_{[p, p+1]}$ is a product. Fix $p \in \mathbb{N}$. Let $j$ be sufficiently large so that $T_{j}$ separates $A_{[p, p+1]}$ from $\mathcal{E}$. Let $T$ be a surface of genus $g=\operatorname{genus}\left(\partial_{\mathcal{E}} C\right)$. Using $\mathrm{Ca}$, CaM], let $F: T \times I \rightarrow Z$ be a homotopy such that $F \mid T \times 1=T_{j}$ and $F(T \times 0) \subset K_{1}$. By Stallings and Waldhausen, after a homotopy of $F$ rel $\partial F$ we can assume that $F^{-1}\left(A_{p} \cup A_{p+1}\right)$ are $\pi_{1}$-injective surfaces in $T \times(0,1)$. See [Wa, p. 60]. Since $F(T \times \partial I) \cap A_{[p, p+1]}=\emptyset$, these surfaces are disjoint from $T \times \partial I$, and by arguing as in [Wa, §3], they are isotopic to surfaces of the form $T \times t, t \in(0,1)$. Therefore, after a further homotopy we can assume that $F^{-1}\left(A_{p} \cup A_{p+1}\right)=T \times B$, where $B \subset(0,1)$ is a finite set of points. Since each $F \mid T \times t$ homologically separates $\mathcal{E}$ from $\partial_{\mathcal{E}} C$, each $F \mid T \times b$ is a degree-1 map onto either $A_{p}$ or $A_{p+1}$ and hence after another homotopy we can assume that for each $b \in B, F \mid T \times b$ is a homeomorphism onto its image. Therefore there exists $b, b^{\prime} \in B$ such that $F \mid T \times\left[b, b^{\prime}\right]$ maps degree- 1 onto $A_{[p, p+1]}$ and the restriction of $F$ to $T \times \partial\left[b, b^{\prime}\right]$ is a homeomorphism. Therefore $F: T \times\left[b, b^{\prime}\right] \rightarrow A_{[p, p+1]}$ is a $\pi_{1}$-injective, degree- 1 map whose restriction to $\partial\left(T \times\left[b, b^{\prime}\right]\right)$ is a homeomorphism onto $\partial A_{[p, p+1]}$. Since both the domain and range are irreducible, such a map is homotopic rel boundary to a homeomorphism, by Waldhausen Wa].

\section{Remarks 6.4.}

(1) In the presence of an escaping sequence of CAT $(-1)$ surfaces, hyperbolic surface interpolation and the bounded diameter lemma is all the hyperbolic geometry needed to establish tameness.

(2) This argument makes crucial use of the fact that the homotopy $F$ is supported in $Z$ and each $A_{i}$ is incompressible in $Z$.

Proof of Theorem 0.4. It suffices to consider the case that $N$ is orientable, since it readily follows using [Tu, that $N$ is tame if and only if its orientable cover is tame. If $\mathcal{E}$ is geometrically finite, then by $[\mathrm{EM}] \mathcal{E}$ is tame. Now assume that $\mathcal{E}$ is geometrically infinite. Theorem 0.9 provides us with a collection $\left\{T_{i}\right\}$ which satisfies the Tameness Criteria (1)-(4). Now apply Theorem 6.3.

Proof of Theorem 0.2 . It suffices to prove Theorem 0.2 for the geometrically infinite ends of orientable manifolds. It follows from Theorems 0.9 and 0.4 that $\mathcal{E}$ is topologically of the form $T \times[0, \infty)$, where $T$ is a surface of genus $g$. Theorem 0.9 
provides for us a sequence $\left\{T_{i}\right\}$ of surfaces satisfying the Tameness Criteria (1)-(4). Since for $i$ sufficiently large $T_{i} \subset T \times[0, \infty)$ and homologically separates $T \times 0$ from $\mathcal{E}$, it follows that the projection $T_{i}$ to $T \times 0$ is a degree 1 map of a genus $g$ surface to itself and hence is homotopic to a homeomorphism.

\section{The PARABOLIC CASE}

Thanks to the careful expositions in $[\mathrm{Bo}, \mathrm{Ca}]$ and $[\mathrm{So}]$ it is now routine to obtain general theorems in the presence of parabolics from the corresponding results in the parabolic free case.

We now give the basic definitions and provide statements of our results in the parabolic setting.

The following is well known; e.g., see $\mathrm{Ca}$ for an expanded version of more or less the following discussion. Let $N$ be a complete hyperbolic 3-manifold. Then for sufficiently small $\epsilon$, the $\epsilon$-thin part, $N_{\leq \epsilon}$ of $N$ is a union of solid tori (Margulis tubes), rank-1 cusps and rank-2 cusps. Let $N_{\geq \epsilon}$ denote $N \backslash \operatorname{int}\left(N_{\leq \epsilon}\right)$. The space $N_{0}^{\epsilon}=N_{\geq \epsilon} \cup$ Margulis tubes is called the neutered space of $N$, though we often drop the $\epsilon$. The parabolic locus $\partial N_{0}^{\epsilon}=P^{\epsilon}$ (usually just denoted $P$ ) is a finite union of tori $T_{1}, \cdots, T_{m}$ and open annuli $A_{1}, \cdots A_{n}$. Each annulus $A_{i}$ is of the form $S^{1} \times \mathbb{R}$ such that for $t \in \mathbb{R}$, each $S^{1} \times t$ bounds a standard 2-dimensional cusp in $N_{\leq \epsilon}$. Let $N_{\leq \epsilon}^{0}$ denote the cusp components of $N_{\leq \epsilon}$. By [Mc], $N$ has a compact core $C \subset N_{0}$ which is also a core of $N_{0}$ and the restriction to each component $P^{\prime}$ of $P$ is a core of $P^{\prime}$. Such a core for $N_{0}$ is called a relative core. In particular if $P^{\prime}$ is an annulus, then we can assume that $C \cap P^{\prime}=S^{1} \times[t, s]$. By Bonahon [Bo, the ends of $N_{0}$ are in 1-1 correspondence with components of $\partial C / P$. If $N=\mathbb{H}^{3} / \Gamma$, then an end of $N_{0}$ is geometrically finite if it has a neighborhood disjoint from $C(\Gamma) / \Gamma$, the convex core of $N$. Such an end has an exponentially flaring geometry similar to that of a geometrically finite end of a parabolic free manifold. The end $\mathcal{E}$ of $N_{0}$ is topologically tame if it is a relative product, i.e., if there is a compact surface $S$ and an embedding $S \times[0, \infty) \rightarrow N_{0}$ which parametrizes $\mathcal{E}$. If $U$ is a neighborhood of $\mathcal{E}$, then by passing to a smaller neighborhood we can assume that $U \cap A_{i}$ is either $\emptyset$ or of the form $S^{1} \times(t, \infty)$ or $S^{1} \times((-\infty, s) \cup(t, \infty))$. Adding the corresponding 2-dimensional cusps to $S^{1} \times$ pts., we obtain $U_{P}$, the parabolic extension of $U$. So if $\mathcal{E}$ is topologically tame, $U_{P}$ is topologically $S^{P} \times[0, \infty)$, where $S^{P}$ is topologically $\operatorname{int}(S)$ and geometrically $S$ with cusps added.

Following [Bo] and $\mathrm{Ca}$ ] we say that the end $\mathcal{E}$ of $N_{0}$ is simply degenerate if it is topologically tame, has a neighborhood $U$ with a sequence $f_{i}: S^{P} \rightarrow U_{P}$ such that $f_{i}$ induces a $\operatorname{CAT}(-1)$ structure on $S^{P}$, the $f_{i}$ 's eventually miss given compact sets and each $f_{i}$ is properly homotopic in $U_{P}$ to a homeomorphism of $S^{P}$ onto $S^{P} \times 0$. We say that $\mathcal{E}$ is geometrically tame if it is simply degenerate or geometrically finite. The manifold $N$ is geometrically tame if each end of $N_{0}$ is geometrically tame.

Francis Bonahon showed that if $\epsilon$ is sufficiently small, then an end $\mathcal{E}$ of $N_{0}^{\epsilon}$ is geometrically infinite if and only if there exists a sequence $\Delta=\left\{\delta_{i}\right\}$ of closed geodesics lying in $N_{0}^{\epsilon}$ and exiting $\mathcal{E}$.

We can now state the general version of the results stated in the introduction.

Theorem 7.1. Let $N$ be a complete hyperbolic 3-manifold with finitely generated fundamental group with neutered space $N_{0}$. The end $\mathcal{E}$ of $N_{0}$ is simply degenerate if there exists a sequence of closed geodesics exiting the end. 
Theorem 7.2. A complete hyperbolic 3-manifold with finitely generated fundamental group is geometrically tame.

Theorem 7.3. If $N$ is a complete hyperbolic 3-manifold with finitely generated fundamental group, then each end of $N_{0}$ is topologically tame. In particular, each end of $N$ is topologically tame.

Theorem 7.4. If $N=\mathbb{H}^{3} / \Gamma$ is a complete hyperbolic 3-manifold with finitely generated fundamental group, then the limit set $L_{\Gamma}$ of $\Gamma$ is either $S_{\infty}^{2}$ or has Lebesgue measure zero. If $L_{\Gamma}=S_{\infty}^{2}$, then $\Gamma$ acts ergodically on $S_{\infty}^{2}$.

Theorem 7.5 (Classification Theorem). If $N$ is a complete hyperbolic 3-manifold with finitely generated fundamental group, then $N$ is determined up to isometry by its topological type, its parabolic structure, the conformal boundary of $N_{0}$ 's geometrically finite ends and the ending laminations of $N_{0}$ 's geometrically infinite ends.

Theorem 7.6 (Density Theorem). If $N=\mathbb{H}^{3} / \Gamma$ is a complete finitely generated 3-manifold with finitely generated fundamental group, then $\Gamma$ is the algebraic limit of geometrically finite Kleinian groups.

Theorem 7.7. Let $N$ be a complete hyperbolic 3-manifold with finitely generated fundamental group and with associated neutered space $N_{0}$. Let $\mathcal{E}$ be an end of $N_{0}$ with relative compact core $C$. Let $S$ be a compact surface with the topological type of $\delta_{\mathcal{E}} C$, the component of the frontier of $C$ which faces $\mathcal{E}$. Let $U_{\mathcal{E}}$ denote a parabolic extension of a neighborhood of $\mathcal{E}$. If there exists a sequence of closed geodesics exiting $\mathcal{E}$, then there exists a sequence $\left\{S_{i}\right\}$ of proper $C A T(-1)$ surfaces in $U_{P}$ homeomorphic to $\operatorname{int}(S)$ which eventually miss every compact set and such that each $S_{i} \cap N_{0}$ homologically separates $C$ from $\mathcal{E}$. Furthermore, if $S_{i} \cap N_{0}$ lies to the $\mathcal{E}$-side of $C$, then no accidental parabolic $\alpha \subset S_{i} \cap N_{0}$ can be homotoped into a cusp via a homotopy disjoint from $C$.

\section{Remarks 7.8 .}

(1) Theorem 7.3 has been independently proven by Agol Ag.

(2) Theorem 7.7 is the main technical result of this section and at the end of this section we will deduce from it Theorems 7.1 and 7.3 .

(3) Theorem 7.1 implies Theorem 7.2 as follows. A complete hyperbolic 3manifold is geometrically tame if each end of $N_{0}$ is either geometrically finite or simply degenerate. By definition, ends of $N_{0}$ are either geometrically finite or geometrically infinite. Using Bonahon's characterization of geometrically infinite ends and Theorem 7.1 it follows that geometrically infinite ends are simply degenerate.

(4) Theorem[7.4 immediately follows from Theorem 7.2 by the work of Thurston T] and Canary [a]. It also follows from $\mathrm{Ca}$ that the various results of [Ca, §9] hold for $N$.

(5) Theorem 7.3 is the last step needed to prove the monumental classification theorem, the other parts being established by Alhfors, Bers, Kra, Marden, Maskit, Mostow, Prasad, Sullivan, Thurston, Minsky, Masur-Minsky, Brock-Canary-Minsky, Ohshika, Kelineidam-Souto, Lecuire, Kim-Lecuire-Ohshika, Hossein-Souto and Rees. See [Mi] and BCM].

(6) The Density Theorem was conjectured by Bers, Sullivan and Thurston. Theorem 7.3 is one of very many results, many of them recent, needed to 
build a proof. Major contributions were made by Alhfors, Bers, Kra, Marden, Maskit, Mostow, Prasad, Sullivan, Thurston, Minsky, Masur-Minsky, Brock-Canary-Minsky, Ohshika, Kelineidam-Souto, Lecuire, Kim-Lecuire-Ohshika, Hossein-Souto, Rees, Bromberg and Brock-Bromberg.

(7) The rest of this section is devoted to proving Theorems 7.7, 7.1 and 7.3

Given the manifold $N$ with neutering $N_{0}$ and end $\mathcal{E}$ of $N_{0}$ we explain how to find a relative end-manifold $M$ containing $\mathcal{E}$.

Definition 7.9. If $A$ is a cod-0 submanifold of a manifold with boundary, then the frontier $\delta A$ of $A$ is the closure of $\partial A \backslash \partial M$. If $(R, \partial R) \subset\left(N_{0}, P\right)$ is a mapped surface (resp. $R$ is a properly mapped surface in $N$ whose ends exit the cusps), then a $P$-essential annulus for $R$ is annulus (resp. half-open annulus) $A$ with one component mapped to an essential simple curve of $R$ which cannot be homotoped within $R$ into $\partial R$ (resp. an end of $R$ ) and another component (resp. the end of A) mapped into $P$ (resp. properly mapped into a cusp). Let $C_{0}$ be a 3 -manifold relative core of $N_{0}$. Using $\mathrm{Mc}$ we can assume that $C_{0}$ is of the form $H_{0} \cup 1$ handles where $P_{0}=C_{0} \cap P=H_{0} \cap P$ is a core of $P$ consisting of annuli and tori and $H_{0}$ is a compact 3-manifold with incompressible frontier. Furthermore, $\delta H_{0}$ has no $P$-essential annuli disjoint from $\operatorname{int}\left(H_{0}\right)$. Define $\delta_{\mathcal{E}} C_{0}$ to be the component of $\delta C_{0}$ which faces $\mathcal{E}$ and $\delta_{\mathcal{E}} H_{0}$ to be the components of $\delta H_{0}$ which face $\mathcal{E}$. Define $M$ to be the closure of the component of $N_{0}$ split along $\delta_{\mathcal{E}} H_{0}$ which contains $\mathcal{E}$. Define $\partial_{p} M=P \cap M$ and $\partial_{h} M=\delta_{\mathcal{E}} H_{0}$. We call $M$ a relative end-manifold. By construction $\partial_{h} M$ has no $P$-essential annuli lying in $M$. By slightly thickening $\partial_{h} M$ and retaining the 1-handles of $C_{0} \cap M$ we obtain a core $C$ of $M$. If $W$ is a codimension- 0 submanifold of $M$, then $\partial_{p} W$ (resp. $\partial_{h} W$ ) denotes $W \cap \partial_{p} M$ (resp. $\left.W \cap \partial_{h} M\right)$.

By passing to the $\pi_{1}(M)$ cover of $N$ we reduce to the case that in : $M \rightarrow N_{0}$ is a homotopy equivalence; furthermore, for each component $R$ of $\partial_{h} M$, the inclusion of $R$ into the corresponding component of $N_{0} \backslash \operatorname{int}(M)$ is a homotopy equivalence.

By passing to a subsequence we can assume that $\Delta=\left\{\delta_{i}\right\}$ is a collection of geodesics escaping $\mathcal{E}$ and is weakly 1000-separating. As in Lemma 5.5 in [Ca] we slightly perturb the hyperbolic metric in the 1-neighborhood of $\Delta$ to a metric $\mu$ such that for each $i, \delta_{i}$ is $\epsilon$-homotopic to a simple geodesic $\gamma_{i}$ and $\mu$ has pinched negative curvature in $(-1.01,-.99)$ and is 1.01-bilipshitz equivalent to the hyperbolic metric. Let $\Gamma$ be the resulting collection of simple closed curves.

Lemma 7.10. Let $M$ be a relative end-manifold in the complete hyperbolic 3manifold $N$. Given a sequence $\Gamma$ of homotopically essential closed curves we can pass to an infinite subsequence also called $\Gamma$ which is the disjoint union $\gamma_{1} \cup \gamma_{2} \cup \gamma_{3} \cup$ ... where $\gamma_{1}$ has finitely many components and the other $\gamma_{i}$ 's have one component. If $\Gamma_{i}$ denotes $\bigcup_{j=1}^{i} \gamma_{j}$, then there exists a manifold $\mathcal{W}$ open in $M$, exhausted by a sequence of compact manifolds $\left\{W_{i}\right\}$ with the following properties.

(1) $\mathcal{W}$ is $\pi_{1}$ and $H_{1}$-injective (in $\mathbb{Z}$ and $\mathbb{Z} / 2 \mathbb{Z}$ coefficients) in $M$ and hence in $N$.

(2) For all $i, \partial_{h} W_{1}=\partial_{h} W_{i}$ and is a union of components of $\partial_{h} M$. At most one component of $\partial_{p} W_{1}$ can lie in a component of $\partial_{p} M$. For all $i, \partial_{p} W_{i}$ is a union of essential annuli, each of which contains a component of $\partial_{p} W_{1}$. 
The frontier $\delta W_{i}$ is connected, separates $\Gamma_{i}$ from $\mathcal{E}$ and is 2-incompressible rel $\Gamma_{i}$.

(3) There exists a compact submanifold core $F \subset W_{1}$ of $\mathcal{W}$ such that each $\Gamma_{i}$ can be homotoped into $F$ via a homotopy supported in $W_{i} . \quad F$ is of the form $\left(W_{1} \cap \partial M\right) \times I$ with 1-handles attached to the 1-side. Finally $|\chi(\delta F)| \leq|\chi(\delta C)|$.

Proof. Except for the last inequality, this lemma is just the relative form of that part of Theorem 5.21 which was used to prove Theorem 0.9. Let $J$ be a connected compact set and $V_{1} \subset V_{2} \subset V_{3} \subset \cdots$ an exhaustion of $M$ such that $\partial_{h} M \subset \partial V_{1}$ and the $\partial_{p} V_{i}$ are the tori of $M$ and essential annuli which meet each annular component of $\partial_{p} M$ in exactly one component. Define the relative end-reduction $\mathcal{W}_{J}$ of $J$ to be the manifold exhausted by submanifolds $\left\{W_{i}\right\}$ where $V_{i}$ passes to $W_{i}$ via the operations of compression, 2-handle addition, deletion and isotopy, where the compressions and 2-handle additions are done only to $\delta V_{i}$ and its successors. The same arguments as before show that $\mathcal{W}_{J}$ is both $\pi_{1}$ and $H_{1}$-injective and as before we can define relative notions of end-nonseparable and end-engulfing respectively for finite and locally finite infinite collections of homotopy essential pairwise disjoint closed curves. Similarly, since $\mathcal{W}$ is a relative end-manifold, its core can be taken to be of the form stated in $\S 3$. The last inequality is the relative version of inequality (*) from the proof of Theorem 0.9 .

We now show that each $W_{i}$ is an atoroidal Haken manifold with negative Euler characteristic by showing that every embedded torus incompressible in $W_{i}$ is boundary parallel and some component of $\partial W_{i}$ is not a 2 -sphere. If $T$ is an embedded torus in $N$, then either $T$ cuts off a rank-2 cusp or $T$ is compressible. Therefore, if each component of $\partial W_{i}$ is a torus incompressible in $N$, then $N$ has finite volume and Theorem 7.7 holds. The proof of the Claim of $\S 6$ shows that no component of $\partial W$ is a torus compressible in $N$. Therefore, some component of $\partial W_{i}$ has genus $\geq 2$. Since tori incompressible in $N$ cut off rank-2 cusps, any nonboundary parallel torus $T$ in $W_{i}$ must be compressible in $N$. To show that $T$ is compressible in $W_{i}$, it suffices to show that it is compressible in $W_{i}^{P}$, the parabolic extension of $W_{i}$ in $N$. Now $\partial W_{i}^{P}$ is a finite union of properly embedded surfaces in $N$ which are incompressible in $N \backslash \Gamma_{i}$ and $N$ has pinched negative sectional curvature. The proof of the Claim now applies to show that every embedded torus in $W_{i}^{P}$ which is compressible in $N$ is also compressible in $W_{i}^{P}$.

Lemma 7.11. If $(E, \partial E) \subset\left(N_{0}, P\right)$ is a compact $\Gamma$-minimal surface (possibly nonembedded), then $E$ cannot be homotoped rel $\partial E$ into $P$.

Suppose $f: R \rightarrow N$ is a properly mapped $\Gamma$-minimal surface such that for each $\epsilon>0, f^{-1}\left(N_{0}^{\epsilon}\right)$ is compact and $R$ has no $P$-essential annuli disjoint from $\Gamma$. If $f$ is transverse to $N_{0}^{\epsilon}$, then each component of $R \backslash \operatorname{int}\left(f^{-1}\left(N_{0}^{\epsilon}\right)\right)$ is either a compact disc or a half-open annulus.

Proof. If such a homotopy exists, then the lift $\tilde{E}$ of $E$ to $\mathbb{H}^{3}$ has the property that there exists a closed horoball $H$ with $\partial \tilde{E} \subset \operatorname{int}(H)$ and $E \cap \partial H \neq \emptyset$. This violates the maximum principle.

Therefore if $\sigma$ is a component of $f^{-1}(P)$ which bounds a disc $D$ in $R$, then $f(D) \cap N_{0} \subset P$. If $\sigma$ is essential in $R$, then $\sigma$ can be homotoped into an end of $R$, since there are no $P$-essential annuli for $R$ disjoint from $\Gamma$. Again the maximum 
principle implies that the entire annular region bounded by $\sigma$ is mapped into a component of $N \backslash \operatorname{int}\left(N_{0}\right)$.

If $W \subset N$ is a codimension-0 submanifold, we say that $W$ has standardly embedded cusps if the restriction of $W$ to each Margulis tube neighborhood of a cusp of $N$ is either the entire cusp, or is a finite union of products of the form annulus $\times \mathbb{R}^{+}$ where the product structure is compatible with the product structure on the cusp. If $N$ is obtained from a complete hyperbolic manifold by neutering, then this neutering should restrict to a neutering of $W$.

Here is the parabolic version of Lemma 2.3. The reader may want to refresh their memory by first rereading Lemma 2.3.

Lemma 7.12 (Parabolic construction lemma). Let $\mathcal{E}$ be an end of the complete open orientable irreducible Riemannian 3-manifold $N$ with metric $\mu$, with finitely generated fundamental group, and neutering $N_{0}$ with parabolic locus $P$. Let $W \subset N$ be a submanifold such that $\partial W \cap \operatorname{int}(N)$ separates $W$ from $\mathcal{E}$, and whose ends are standardly embedded cusps in the cusps of $N$. Let $\Delta_{1} \subset N_{0} \backslash \partial W$ be a finite collection of simple closed geodesics with $\Delta=W \cap \Delta_{1}$ a nonempty proper subset of $\Delta_{1}$. Suppose furthermore that $\partial W$ is 2-incompressible rel. $\Delta$ and has no $P$ essential annuli disjoint from $\Delta_{1}$.

Let the Riemannian metric $\mu$ on $N$ agree with a hyperbolic metric outside tubular neighborhoods $N_{\epsilon}\left(\Delta_{1}\right)$ and inside tubular neighborhoods $N_{\epsilon / 2}\left(\Delta_{1}\right)$, having $\Delta_{1}$ as core geodesics, and such that $\mu$ is a metric with sectional curvature pinched between -1.01 and -0.99 .

Let $G$ be a finitely generated subgroup of $\pi_{1}(W)$, and let $X$ be the covering space of $W$ corresponding to $G$. Let $\Sigma$ be the preimage of $\Delta$ in $X$ with $\hat{\Delta} \subset \Sigma$ a subset which maps homeomorphically onto $\Delta$ under the covering projection, and let $B \subset \hat{\Delta}$ be a nonempty union of geodesics. Suppose there exists a properly embedded surface $S \subset X \backslash B$ of finite topological type, whose ends are standard cusps in the cusps of $X$ such that $S$ is 2-incompressible rel. $B$ in $X$ and has no $P$-essential annuli disjoint from $B$, and which separates every component of $B$ from $\partial X$.

Then $\partial W$ can be properly homotoped to a $\Delta_{1}$-minimal surface which, by abuse of notation, we call $\partial W^{\prime}$, and the map of $S$ into $N$ given by the covering projection is properly homotopic to a map whose image $T^{\prime}$ is $\Delta_{1}$-minimal and whose ends exit the cusps of $N$.

Also, $\partial W^{\prime}$ (resp. $\left.T^{\prime}\right)$ can be perturbed by an arbitrarily small perturbation to be an embedded (resp. smoothly immersed) surface $\partial W_{t}$ (resp. $T_{t}$ ) bounding $W_{t}$ with the following properties:

(1) There exists a proper isotopy from $\partial W$ to $\partial W_{t}$ which never crosses $\Delta_{1}$, and which induces a proper isotopy from $W$ to $W_{t}$, and a corresponding deformation of pinched negatively curved manifolds $X$ to $X_{t}$ which fixes $\Sigma$ pointwise.

(2) There exists a proper isotopy from $S$ to $S_{t} \subset X_{t}$ which never crosses $B$, such that $T_{t}$ is the projection of $S_{t}$ to $N$.

(3) Each of the limit surfaces $F \in\left\{\partial W^{\prime}, T^{\prime}\right\}$ relatively exits the manifold as its restriction exits the neutered part. That is, if $\mathcal{C}$ is a rank 1 cusp foliated by totally geodesic 2-dimensional cusps $C \times \mathbb{R}$ perpendicular to the boundary annulus $S^{1} \times \mathbb{R}$, then if the intersection of $F$ with $\partial \mathcal{C}$ is contained in the 
region $S^{1} \times[t, \infty)$, the intersection of $F$ with $\mathcal{C}$ is contained in the region $C \times[t, \infty)$, and similarly if the intersection is contained in $S^{1} \times(-\infty, t]$.

Proof. The essential differences between the statements of Lemma 2.3 and Lemma 7.12 are firstly that the metric in the parabolic case is pinched, so that the geodesics can be chosen to be simple; secondly that the surfaces in question are all properly embedded, and the isotopies and homotopies are all proper; and thirdly that the limit surfaces relatively exit the manifold as their restriction exits the neutered part.

These issues are all minor and do not introduce any real complications in the proof. The only question whose answer might not be immediately apparent is how to perturb the metric $\mu$ to the $g_{t}$ metrics near cusps; it turns out that this is straightforward to do, and technically easier than deformations along geodesics, since the perturbed metrics actually have curvature bounded above by 0 .

We will find an exhaustion of $N$ by increasingly larger neutered spaces $N_{0}^{t}$, each endowed with a metric $g_{t}$, which is obtained from the $\mu$-metric by deforming it along the geodesics $\Delta_{1}$ and along $\partial N_{0}^{t}$. Our $\partial W_{t}$ will restrict to $g_{t}$-area minimizing representatives of the isotopy class of $\partial W \cap N_{0}^{t}$. The convergence and regularity of the limit surface $\partial W^{\prime}$ near the geodesics will proceed exactly as in $\S 1$ and $\S 2$. The convergence and regularity in the cusps will follow from $\S 1$ using the absence of $P$-essential annuli disjoint from $\Delta_{1}$.

To describe the deformed geometry along the cusps, we first recall the usual hyperbolic geometry of the (rank 1 ) cusps. We parameterize a rank 1 cusp $\mathcal{C}$ as $S^{1} \times[1, \infty) \times \mathbb{R}$, where the initial $S^{1} \times[1, \infty)$ factor is a 2 -dimensional cusp $C$. With the hyperbolic metric, the three coordinate vector fields are orthogonal; we denote these by $\frac{\partial}{\partial \theta}$, $\frac{\partial}{\partial z}$ and $\frac{\partial}{\partial y}$ respectively, so that $\theta \in S^{1}, z \in[1, \infty)$ and $y \in \mathbb{R}$. An orthonormal basis in the hyperbolic metric is $z \frac{\partial}{\partial \theta}, z \frac{\partial}{\partial z}, z \frac{\partial}{\partial y}$. Let $h: \mathbb{R}^{+} \rightarrow \mathbb{R}^{+}$ be a monotone increasing function with $h(z)=z$ for $z<1$, and $h(z)=2$ for $z \geq 3$. Then let

$$
h_{t}(z)=\frac{1}{1-t} h((1-t) z)
$$

and define $g_{t}$ on $\mathcal{C}$ to be the metric with orthonormal basis $h_{t}(z) \frac{\partial}{\partial \theta}, h_{t}(z) \frac{\partial}{\partial z}, h_{t}(z) \frac{\partial}{\partial y}$. Notice that the group of Euclidean symmetries of the boundary $\partial \mathcal{C}$ extends to an isometry of $\mathcal{C}$ for the $g_{t}$ metric, for all $t$. In particular, the surface

$$
H_{s}=S^{1} \times[1, \infty) \times s
$$

is totally geodesic for the $g_{t}$ metric and therefore acts as a barrier surface for all $t$.

Moreover, as $t \rightarrow 1$, the $g_{t}$ metrics converge to the hyperbolic metrics on compact subsets, and in fact for every compact $K \subset \mathcal{C}$, there is an $s>0$ such that the $g_{t}$ and the hyperbolic metrics agree for $t \leq s$. Finally, for each $t>0$, the subset $S^{1} \times[3 /(1-t), \infty) \times \mathbb{R} \subset \mathcal{C}$ is isometric to a Euclidean product, for the $g_{t}$ metric, and therefore the surface

$$
F_{t}=S^{1} \times \frac{3}{1-t} \times \mathbb{R}
$$

is totally geodesic for the $g_{t}$ metric and also acts as a barrier surface.

Finally, notice that the $g_{t}$ metrics lift to a family of isometric metrics on $\mathbb{H}^{3}$ and, by the symmetries above, therefore have uniformly pinched sectional curvatures, and are uniformly bilipschitz to the hyperbolic metric in the region bounded away from the cusps by $F_{t}$. 
Let $N_{0}^{t}$ be the neutered space whose boundary consists of the surfaces of type $F_{t}$ constructed above. Endow $N_{0}^{t}$ with the $g_{t}$ metric. Now apply [MSY], as in Lemma 2.3. to the surface $\partial W \cap N_{0}^{t}$ to obtain the surface $\partial W_{t}^{1}$ which is $g_{t}$ least area among all surfaces properly isotopic to $\partial W \cap N_{0}^{t}$. By extending $\partial W_{t}^{1}$ vertically we obtain the surface $\partial W_{t} \subset N$ which is properly isotopic to $\partial W$. As in Lemma 2.3 these surfaces weakly converge geometrically to a surface $\partial W^{\prime}$. We will show that there is a proper isotopy of $\partial W$ to $\partial W^{\prime}$.

Let $N_{0}^{\epsilon}$ denote a fixed neutered space transverse to $\partial W^{\prime}$ and countably many $\partial W_{t}$ 's which converge to $\partial W^{\prime}$. Define $\partial W_{t}^{\epsilon}$ to be $\partial W_{t} \cap N_{0}^{\epsilon}$ together with the disc components of $\partial W_{t} \backslash N_{0}^{\epsilon}$. Since $\operatorname{area}_{g_{t}}\left(\partial W_{t}^{\epsilon}\right)$ is uniformly bounded, and the hyperbolic area form is dominated on all 2-planes by $g_{t}$, the hyperbolic area of $\partial W_{t}^{\epsilon}$ is uniformly bounded. We show that a disc $D$ of $\partial W_{t} \backslash N_{0}^{\epsilon}$ cannot stray too far into the cusp and hence for all $t, \partial W_{t}^{\epsilon} \subset N_{0}^{\eta}$ for some sufficiently small $\eta$. Indeed, the lift $\tilde{D}$ to the universal cover $\tilde{N}$ of $N$ is an embedded disc of uniformly bounded area. If $t$ is very close to 1 , then $d_{\rho}\left(\partial N_{0}^{\epsilon}, \partial N_{0}^{t}\right)=d_{t}>0$. If $x \in \tilde{D}$ and $d_{\rho}\left(x, \partial N_{0}^{\epsilon} \cup N_{0}^{t}\right)=d_{t} / 2$, then $\operatorname{area}_{\rho}\left(\tilde{D} \cap\left(N_{0}^{\epsilon}-N_{0}^{t}\right)\right)>\pi d_{t}^{2} / 4$. Therefore, for $t$ sufficiently large, $\tilde{D}$ and hence $D$ has uniformly bounded $\rho$-diameter.

Therefore, if $\epsilon<\eta$, then the $\partial W_{t}^{\epsilon}$ 's converge weakly to the surface $N_{0}^{\epsilon} \cap W^{\prime}$, which we define to be $\partial W_{\epsilon}^{\prime}$. For $t$ sufficiently large, $\partial W_{t}^{\epsilon}$ and $\partial W_{\epsilon}^{\prime}$ are of the same topological type and very close geometrically. By Lemma 7.11, $\partial W_{\epsilon}^{\prime} \cap N_{0}^{\epsilon}$ has no components which can be homotoped rel boundary into $\partial N_{0}^{\epsilon}$; hence $\partial W_{t}^{\epsilon}$ shares the similar property. Since $\partial W$ has no $P$-essential annuli disjoint from $\Delta_{1}$ it follows that each component of $\partial W_{t}^{\epsilon} \backslash$ int $N_{0}$ can be properly homotoped in $\partial W_{t}^{\epsilon}$ into an end of that surface. Therefore, if some nondisc component of $\partial W_{t}-\operatorname{int} N_{0}^{\epsilon}$ was not a half-open annulus, then one can find a component $E$ of $\partial W_{t} \cap N_{0}^{\epsilon}$ which can be homotoped rel $\partial E$ into $\partial N_{0}^{\epsilon}$, which is a contradiction. Note that $\partial W_{\epsilon}^{\prime}$ is of the same topological type as $\partial W \cap N_{0}$ and and that the $\partial W_{\epsilon}^{\prime \prime s}$ form an exhaustion of $\partial W^{\prime}$. By arguing as in the proof of Lemma 1.25, there exists a homotopy $F: \partial W \times I \rightarrow N$ with the property that $F(\partial W \times 0)=\partial W$, for infinitely many $t<1, F(\partial W \times t)=\partial W_{t}$ and $F(\partial W \times 1)=\partial W^{\prime}$.

If $\partial W^{\prime}$ intersects $\partial \mathcal{C}$ in the subset $S^{1} \times[s, \infty)$ for some $s$, then for $t$ sufficiently large $\partial W_{t}$ must intersect $\partial \mathcal{C}$ in the subset $S^{1} \times[s-\epsilon, \infty)$. Since projection to the barrier surface $H_{s-\epsilon}$ along horoannuli and horotori is area reducing, this implies that $\partial W_{t} \cap \mathcal{C}$ is contained in $S^{1} \times[1, \infty) \times[s-\epsilon, \infty)$, which in turn implies that $W^{\prime} \cap \mathcal{C} \subset S^{1} \times[1, \infty) \times[s, \infty)$. As in Lemma 2.3. the surfaces $\partial W_{t}$ converge on compact subsets to $\partial W^{\prime}$. The main results of $\S 1$ imply that $\partial W^{\prime}$ is $\Delta_{1}$-minimal.

A similar argument proves similar facts about $S_{t}, T_{t}$ and $T^{\prime}$.

Let $G_{i}$ denote $\operatorname{in}_{*}\left(\pi_{1}(F)\right) \subset \pi_{1}\left(W_{i}\right)$. Fix a basepoint $f \in F$. Let $X_{i}$ denote the covering space of $W_{i}$ (based at $f$ ) with group $G_{i}$. The homotopy of $\Gamma_{i}$ into $F$ supported in $W_{i}$ lifts to $X_{i}$, hence provides us with a canonical $\hat{\Gamma}_{i}$ of closed lifts of $\Gamma_{i}$ in 1-1 correspondence with $\Gamma_{i}$. Since $W_{i}$ is an atoroidal Haken manifold with nonzero Euler characteristic, it follows by Thurston that $\operatorname{int}\left(X_{i}\right)$ is topologically tame (see Proposition 3.2 in $\mathrm{Ca}$ ). By Tu2 a compactification $\bar{X}_{i}$ of $\operatorname{int}\left(X_{i}\right)$ extends $\operatorname{int}\left(X_{i}\right) \cup \partial_{h} \hat{F} \cup \partial_{p} \hat{F}$, where $\hat{F}$ is the lift of $F$ to $X_{i}$. Since $\hat{F}$ is a core of $\bar{X}_{i}$ it follows that $\bar{X}_{i}$ is a union of a closed (possibly disconnected or empty) 
orientable surface $\times I$ with 1 -handles attached to the surface $\times 1$ side. Let $\bar{S}_{i}$ denote the unique boundary component of $\bar{X}_{i}$ which is not a closed component of $\hat{F}$. Push $\bar{S}_{i} \backslash \operatorname{int}\left(\hat{F} \cap \partial \bar{X}_{i}\right)$ slightly to obtain a properly embedded surface $\hat{S}_{i} \subset X_{i}$ with $\partial \hat{S}_{i}=\partial \delta \hat{F}$ via a homotopy disjoint from $\hat{\Gamma}_{i}$. Being connected with the same Euler characteristic and the same number of boundary components, $\hat{S}_{i}$ is of the same topological type as $\delta F$. Let $\hat{Z}_{i}^{\prime \prime} \subset \bar{X}_{i}$ be the compact region with frontier $\hat{S}_{i}$. Let $\chi:=\left|\chi\left(\hat{S}_{i}\right)\right|=|\chi(\delta F)|$. Define $\partial_{p} X_{i}$ and $\partial_{h} X_{i}$ to be the respective preimages of $\partial_{p} W_{i}$ and $\partial_{h} W_{i}$.

Let $W_{i}^{\prime}$ denote $W_{i}$ together with the components of $N_{0} \backslash \operatorname{int}(M)$ which hit $\partial W_{1}$. Let $Y_{i}$ be the cover of $W_{i}^{\prime}$ with $\pi_{1}\left(Y_{i}\right)=G_{i}$. As in the parabolic free case, $X_{i}$ naturally embeds in $Y_{i}$ and the inclusion is a homotopy equivalence. Define $\partial_{p} Y_{i}$ to be the preimage of $\partial_{p} W_{i}^{\prime}$. Note that $\delta W_{i}$, the frontier of $W_{i} \subset M$, equals $\delta W_{i}^{\prime}$, the frontier of $W_{i}^{\prime}$ in $N_{0}$.

If possible compress $\hat{Z}_{i}^{\prime \prime}$ along $\delta \hat{Z}_{i}^{\prime \prime}=\hat{S}_{i}$ via compressions that hit $\hat{\Gamma}_{i}$ at most once. Continue in this manner to obtain the region $\hat{Z}_{i}^{\prime}$ whose frontier is 2-incompressible rel $\hat{B}^{i}:=\hat{\Gamma}_{i} \backslash\left\{\gamma_{i_{1}}, \cdots, \gamma_{i_{m}}\right\}$, where both $m$ and $\left|\chi\left(\delta \hat{Z}_{i}^{\prime}\right)\right| \leq \chi$. Since $X_{i} \backslash \hat{\Gamma}_{i}$ is irreducible, we can assume that no component of $\partial \hat{Z}_{i}^{\prime}$ is a 2-sphere.

Before we shrinkwrap $\delta W_{i}^{\prime}$ and $\delta \hat{Z}_{i}^{\prime}$ we need to annulate them, i.e., compress them along essential annuli into $P$ and $\partial_{p} Y_{i}$. Geometrically we are eliminating accidental parabolics so that we can invoke Lemma 7.12 .

Let $L_{1}, \cdots, L_{k}$ be a maximal collection of pairwise disjoint, embedded, essential annuli in $N_{0}$ disjoint from $\Gamma_{i}$ such that for each $j, \partial L_{j}$ has one component on $\delta W_{i}^{\prime}$ and one component on $P$. Furthermore assume that $\operatorname{int}\left(L_{i}\right) \cap \delta W_{i}^{\prime}=\emptyset$. Now annulate $\delta W_{i}^{\prime}$ along each $L_{i}$ to obtain the surface $\delta W_{i}^{L}$. So if $L_{i}$ lies to the outside of $W_{i}^{\prime}$, then the effect on $W_{i}$ is to add $N\left(L_{i}\right)$. If $L_{i} \subset W_{i}^{\prime}$ and $L_{i} \times I$ is a product neighborhood, then this annulation deletes $L_{i} \times \operatorname{int}(I)$ from $W_{i}^{\prime}$. There are no $P$-essential annuli for $\delta W_{i}^{L}$ disjoint from $\Gamma_{i}$, and $\partial W_{i}^{L}$ is 2-incompressible rel $\Gamma_{i}$. Indeed, since $\delta W_{i}^{L}$ is embedded and 2-incompressible, we need only consider embedded $P$-essential annuli, by the generalized loop theorem. The modification $W_{i}^{\prime} \rightarrow W_{i}^{L}$ induces a modification of $Y_{i}$ as follows. If $L_{j}$ annulates $W_{i}^{\prime}$ to the outside, then enlarge $Y_{i}$ in the natural way. This will enlarge the parabolic boundary $\partial_{p} Y_{i}$. If $\delta W_{i}^{\prime}$ gets annulated to the inside, then do not change $Y_{i}$. By abuse of notation, we relabel the space obtained from $Y_{i}$ as $Y_{i}$. Let $W_{i}^{l}$ denote $W_{i}$ modified only along outer $P$-essential annuli. Note that $Y_{i}$ is a covering space of $W_{i}^{l}$. In like manner, annulate $\delta \hat{Z}_{i}^{\prime}$ along a maximal collection of pairwise disjoint annuli which are disjoint from $\hat{B}_{i}$. Let $\hat{Z}_{i} \subset Y_{i}$ denote the result of annulating $\hat{Z}_{i}^{\prime}$. Note that $\hat{Z}_{i}$ can be constructed so that there are no $\partial_{p} Y_{i}$-essential annuli for $\delta \hat{Z}_{i}$ disjoint from $\hat{B}_{i}$.

Now fix $i$. Let $\gamma \in \Gamma \backslash W_{i}^{L}$. Apply Lemma 7.12 using the following dictionary between our setting and the setting of Lemma $7.12, \delta \hat{Z}_{i}$ corresponds to the surface $S, W_{i}^{L}$ corresponds to $W, Y_{i}$ corresponds to $X, \gamma \cup \Gamma_{i}$ corresponds to $\Delta_{1}, \Gamma_{i}$ corresponds to $\Delta$, and $\hat{B}^{i}$ corresponds to $B$. We conclude that if $S^{i}$ denotes the projection of $\delta \hat{Z}_{i}$ into $N$, then $S^{i}$ is homotopic to a $\mathrm{CAT}(-1)$ surface $T^{i}$ with the following properties. The surface $T^{i}$ is homotopic to a surface $P^{i} \subset W_{i}^{\text {new }}$, where $W_{i}^{\text {new }}$ is isotopic to $W_{i}^{l}$ and $P^{i}$ lifts to an embedded surface $\hat{P}^{i} \subset Y_{i}^{\text {new }}$, where $Y_{i}^{\text {new }}$ is the corresponding cover of $W_{i}^{\text {new }}$. The isotopy of $W_{i}^{\text {new }}$ to $W_{i}^{l}$ induces 
a deformation of spaces $Y_{i}^{\text {new }}$ to $Y_{i}$ which fixes $\hat{B}^{i}$ pointwise. Futhermore, $\hat{P}^{i}$ is isotopic to the corresponding $\delta \hat{Z}_{i}$ via an isotopy disjoint from $\hat{B}^{i}$. Given $\epsilon>0$, the $P^{i}$ can be chosen so that the homotopy of $T^{i}$ to $P^{i}$ restricted to $N_{0}^{\epsilon}$ lies in an $\epsilon$-neighborhood of $P^{i} \cap N_{0}^{\epsilon}$. By abuse of notation we will view $\hat{P}^{i}$ as bounding the region $\hat{Z}_{i} \subset Y_{i}^{\text {new }}$ and we will drop the superscripts "new", etc.

Let $\left\{\alpha_{i}\right\}$ be a locally finite collection of embedded proper rays in $N_{0}$ to $\mathcal{E}$ emanating from $\left\{\gamma_{i}\right\}$.

Let $\pi: Y_{i} \rightarrow N$ be the composition of the covering map to $W_{i}^{l}$ and inclusion. Let $B^{i}=\pi\left(\hat{B}^{i}\right)$. If $b \in B^{i}$ and is disjoint from $N\left(P^{i}, 1\right)$, then some component of $T^{i}$ homologically separates $b$ from $\mathcal{E}$. Indeed if $\alpha_{b}$ is the ray from $b$ to $\mathcal{E}$, then $\pi^{-1}\left(\alpha_{b}\right) \cap \hat{Z}_{i}$ is a finite union of compact segments. If both endpoints lie in $\partial Y_{i}$, then it contributes nothing to the algebraic intersection number $\left\langle\alpha_{b}, P^{i}\right\rangle$. Otherwise it has one endpoint in $\pi^{-1}\left(\alpha_{b}\right)$ and one in $\partial Y_{i}$ and hence contributes +1 . Therefore

$$
\left\langle\alpha_{b}, T^{i}\right\rangle=\left\langle\alpha_{b}, P^{i}\right\rangle>0 .
$$

Since $\left|B^{i}\right| \rightarrow \infty$, the $B^{i}$ 's are weakly 1000 separating and the $T^{i}$ 's have uniformly bounded area, it follows that for $i$ sufficiently large, some $b \in B^{i} \backslash B^{j_{i}}$ is disjoint from $N\left(P^{i}, 1\right)$, where $j_{i}<i$ and $\lim _{i \rightarrow \infty} j_{i}=\infty$. Therefore some subsequence of components of $\left\{T^{i}\right\}$ exits $\mathcal{E}$.

By reducing $\epsilon$, if necessary, we can assume that $\partial N_{0}^{\epsilon}$ is transverse to all the $T^{i}$,s. By Lemma 7.11 for each $i$, each component of $T^{i} \cap\left(N \backslash \operatorname{int}\left(N_{0}\right)\right)$ is either a disc or a half-open annulus. Therefore, the restriction of each component of $T^{i}$ to $N_{0}$ is a connected surface.

Lemma 7.13. Let $M$ be a relative end-manifold with core $C$ of the form $\partial_{h} M \times$ $I \cup 1$-handles. Let $Z$ denote the closure of $M-C$ with $\partial_{p} Z=\partial_{p} M \cap Z$ and $\partial_{\mathcal{E}} Z=\partial_{\mathcal{E}} C$.

(1) $Z$ is Thurston norm-minimizing in $H_{2}\left(Z, \partial_{p} Z\right)=\mathbb{Z}$.

(2) If $R$ is a Thurston norm-minimizing surface (in either the singular or embedded norms), representing $\left[\partial_{\mathcal{E}} Z\right] \in H_{2}\left(Z, \partial_{p} Z\right)$, then for each component $Q$ of $\partial_{p} Z$ we have $|R \cap Q|=1$. In particular, $R$ has no $P$-essential annuli in $Z$.

Corollary 7.14. Let $N$ be a complete hyperbolic 3-manifold with neutering $N_{0}$ and relative core $C$ for $N_{0}$. Let $R \subset N_{0}-C$ be a primitive Thurston norm-minimizing surface representing an element of $H_{2}\left(N_{0}-C, \partial_{p} N_{0}\right)$. Then every homotopy of an accidental parabolic of $R$ into $\partial_{p} N_{0}$ must cross $C$.

Proof of Lemma. The proof of (1) is similar to that of Lemma 6.1. Recall that since $C$ is a core, the inclusion $\left(\partial_{\mathcal{E}} Z, \partial \partial_{\mathcal{E}} Z\right) \rightarrow\left(Z, \partial_{p}(Z)\right)$ is an isomorphism.

Now let $R$ be a possibly singular Thurston norm-minimizing surface representing $\left[\partial_{\mathcal{E}} Z\right]$. By [G1], $\chi(R)=\chi\left(\partial_{\mathcal{E}} Z\right)$, so if $R$ hits $\partial_{p}(Z)$ in extra components, then $\operatorname{genus}(R)<\operatorname{genus}\left(\partial_{\mathcal{E}} Z\right)$. Let $S \subset Z$ be an embedded surface representing $\left[\partial_{\mathcal{E}} Z\right]$ such that $R$ lies in $\operatorname{int}\left(Z^{\prime}\right)$, where $Z^{\prime}$ is the compact submanifold cut off by $S$. If $\left\{a_{1}, \cdots, a_{2 g}\right\}$ is a basis of cycles in $H_{1}\left(\partial_{\mathcal{E}} Z, \partial \partial_{\mathcal{E}} Z\right)$ which are disjoint from $\partial \partial_{\mathcal{E}} Z$, then there exist surfaces $A_{1}, \cdots, A_{2 g}$ with $\partial A_{i} \subset \partial_{\mathcal{E}} Z \cup S$ and $\left[A_{i} \cap \partial_{\mathcal{E}} Z\right]=n_{i}\left[a_{i}\right] \in$ $H_{1}\left(\partial_{\mathcal{E}} Z, \partial \partial_{\mathcal{E}} Z\right)$ where $n_{i} \neq 0$. For each $i$, let $\left[A_{i} \cap S\right]=b_{i} \in H_{1}(S, \partial S)$. Since the subgroup of $H_{1}(S, \partial S)$ which restricts trivially to $\partial S$ is of rank $<2 g$, it follows that $b_{1}, \cdots, b_{2 g}$ are linearly dependent. This implies that the inclusion $\left(\partial_{\mathcal{E}} Z, \partial \partial_{\mathcal{E}} Z\right) \rightarrow$ 
$\left(Z, \partial_{p}(Z)\right)$ is not $H_{1}$-injective, a contradiction. If $R$ had a $P$-essential annulus, then we can construct a norm-minimizing surface $R^{\prime}$ with $\left|\partial R^{\prime}\right|=|\partial R|+2$.

We next show that if some component $T$ of $T^{i}$ has the property that $T \cap N_{0}$ homologically separates $C$ from $\mathcal{E}$, then $T=T^{i}$ is homeomorphic to $\partial_{\mathcal{E}} C$ and represents the class $\left[\partial_{\mathcal{E}} C\right] \in H_{2}\left(N_{0}, P\right)$. Suppose that $\left[T \cap N_{0}\right]=n\left[\partial_{\mathcal{E}} C\right] \in H_{2}\left(N_{0}, P\right)$. By Lemma 17.11, after a homotopy supported in a small neighborhood of the cusps we can push the disc components of $T \cap N \backslash \operatorname{int}\left(N_{0}\right)$ into $N_{0}$ and get $|\chi(F)| \geq \chi \geq$ $|\chi(T)|=\left|\chi\left(T \cap N_{0}\right)\right|$. By Lemma 7.10, $\mid \chi\left(\partial_{\mathcal{E}} C|\geq| \chi(F) \mid\right.$. On the other hand,

$$
|\chi(T)| \geq x_{s}\left(n\left[\partial_{\mathcal{E}} C\right]\right)=x\left(n\left[\partial_{\mathcal{E}} C\right]\right)=n x\left(n\left[\partial_{\mathcal{E}} C\right]\right)=n\left|\chi\left(\partial_{\mathcal{E}} C\right)\right|
$$

where the $x$ and $x_{s}$ respectively denote the Thurston and singular Thurston norms and the inequality is, by definition, the first equality by G1], the second by [T2] and the third by Lemma 7.13. The only possibility is that $n=1$ and $|\chi(T)|=$ $\left|\chi\left(\partial_{\mathcal{E}} C\right)\right|=\chi$ and hence $T=T^{i}$. By Lemma 7.13, $T$ and $\partial_{\mathcal{E}} C$ have the same number of boundary components and hence $T=T^{i}$ is homeomorphic to $\partial_{\mathcal{E}} C$. In particular no compressions or annulations occurred to $\hat{S}^{i}$.

We claim that the sequence $\left\{T^{i} \cap N_{0}\right\}$ exits $N_{0}$. Otherwise, there exists an $m$ with $1 \leq m \leq \chi$, a subsequence $T^{i_{1}}, T^{i_{2}}, \cdots$ and a compact connected submanifold $K_{1} \subset N_{0}$ such that $C \subset K_{1}$ and for each $j, m$ components of $T^{i_{j}}$ nontrivially intersect $K_{1}$ and if $R^{i_{j}}$ are the components of $T^{i_{j}}$ which miss $K_{1}$, then $R^{i_{j}} \cap N_{0}$ is an exiting sequence. Since each component $T$ of $T^{i_{j}}$ has $T \cap N_{0}$ connected, it follows from the bounded diameter lemma that there exists a compact set $K_{2}$ such that for all $j$, if $T$ is a component of $T^{i_{j}}$ with $T \cap K_{1} \neq \emptyset$, then $T \cap N_{0} \subset K_{2}$. Let $N$ be so large that $\gamma_{N} \cap \alpha_{N} \cap N_{2}\left(K_{2}\right)=\emptyset$ and $\gamma_{N} \subset B_{i_{j}}$ for infinitely many values of $j$. Let $\beta_{N}$ be a path from $\gamma_{N}$ to $K_{2}$. Since $R^{i_{j}}$ exits $N_{0}$ it follows that for $j$ sufficiently large $\left(\gamma_{N} \cup \beta_{N}\right) \cap N_{2}\left(T^{i_{j}}\right)=\emptyset$. This implies that some component $T$ of $T^{i_{j}}$ homologically separates $\gamma_{N}$ and hence $C$ from $\mathcal{E}$. Therefore $|\chi(T)|=\chi$. Since $T \cap \alpha_{N} \neq \emptyset$, this implies that $T \subset R^{i_{j}}$ and hence $m=0$, which is a contradiction.

Since the sequence $\left\{T^{i} \cap N_{0}\right\}$ exits $N_{0}$ it follows from the previous paragraphs that for $i$ sufficiently large, $T^{i}$ is homeomorphic to $\hat{S}^{i}$, and $T^{i} \cap N_{0}$ represents the class $\left[\partial_{\mathcal{E}} C\right] \in H_{2}\left(N_{0}, P\right)$. Since $\left\{T^{i}\right\}$ exits $\mathcal{E}$, if $B$ is a cusp of $N$ parametrized by $S^{1} \times[0, \infty) \times \mathbb{R}$, then by Proposition 7.12 , given $n \in \mathbb{R}, T^{i} \cap B \subset S^{1} \times[0, \infty) \times$ $(n, \infty)$.

Remark 7.15. Since for $i$ sufficiently large, $T^{i}$ is of topological type of $\partial_{\mathcal{E}} C$, it follows a posteriori that no compressions or annulations occurred in the passage from $\bar{S}^{i}$ to $\partial \hat{Z}_{i}$. This mirrors the similar phenomena seen in the proofs of Canary's theorem and Theorem 0.9 .

Proof of Theorem 7.3 . Tameness of the ends of $N_{0}$ follows as in the proof of Theorem 0.4. In particular, if the end $\mathcal{E}$ of $N_{0}$ is not geometrically finite, then by applying the proof of [So, Theorem 2] to $\left\{T^{i}\right\}$ (with the disc components of $\left\{T^{i}\right\} \cap$ (cusps) pushed into $N_{0}$ ) it follows that $\mathcal{E}$ is tame. Alternatively, as in the proof that Criteria (1)-(4) imply tameness, we can use the hyperbolic surface interpolation technique and basic 3 -manifold topology to prove that $\mathcal{E}$ is tame. Finally, tameness of $N_{0}$ implies tameness of $N$. 
Proof of Theorem 7.1. It suffices to prove Theorem 7.1 for orientable manifolds which have the homotopy type of a relative end-manifold. It follows from Theorems 7.7 and 7.3 that a parabolic extension $U_{P}$ of a neighborhood $U$ of $\mathcal{E}$ is topologically of the form $\operatorname{int}(T) \times[0, \infty)$, where $T$ is a surface homeomorphic to $\partial_{\mathcal{E}} C$ and $C$ is a core of $N_{0}$. By Proposition 7.12, if $\left(T^{i} \cap \partial N_{0}\right) \subset \operatorname{int}(T) \times[t, \infty)$, then $T^{i} \backslash \operatorname{int} N_{0} \subset$ $\operatorname{int}(T) \times[t, \infty)$. Therefore $\left\{T^{i}\right\}$ exits compact sets in $\operatorname{int}(T) \times[0, \infty)$. Since for $i$ sufficiently large, $T^{i}$ is properly immersed in $\operatorname{int}(T) \times[0, \infty)$ and homologically separates $\operatorname{int}(T) \times 0$ from $\mathcal{E}$, it follows that the projection $T_{i}$ to $\operatorname{int}(T) \times 0$ is a proper degree-1 map of a surface of finite type to itself and hence is properly homotopic to a homeomorphism.

\section{ACKNOWLEDGEMENTS}

The first author is grateful to Nick Makarov for some useful analytic discussions. The second author is grateful to Michael Freedman for many long conversations in Fall 1996 which introduced him to the Tame Ends conjecture. He thanks Francis Bonahon, Yair Minsky and Jeff Brock for their interest and helpful comments. Part of this research was carried out while he was visiting Nara Women's University, the Technion and the Institute for Advanced Study. He thanks them for their hospitality. We thank the referees for their many thoughtful suggestions and comments.

\section{REFERENCES}

[Ag] I. Agol, Tameness of hyperbolic 3-manifolds, eprint math.GT/0405568.

[A1] L. Ahlfors, Finitely generated Kleinian groups, Amer. J. Math. 86 (1964), 413-429. MR.0167618 (29:4890)

[A2] Fundamental polygons and limit point sets of Kleinian groups, Proc. Nat. Acad. Sci. 55 (1966), 251-254. MR0194970(33:3175)

[Ath] I. Athanasopoulos, Coincidence set of minimal surfaces for the thin obstacle, Manuscripta Math. 42 (1983), no. 2-3, 199-209. MR0701203 (85a:49048)

[B] W. Ballmann, Lectures on spaces of nonpositive curvature, Birkhäuser, DMV seminar 25 (1995). MR1377265 (97a:53053)

[Be] L. Bers, Local behavior of solutions of general elliptic equations, Comm. Pure. Appl. Math. 8 (1955), 473-496. MR0075416 (17:743a)

[Bo] F. Bonahon, Bouts des variétés de dimension 3, Annals of Math. 124 (1986), 71-158. MR.0847953 (88c:57013)

[BH] M. Bridson \& A. Haefliger, Metric spaces of non-positive curvature, Springer-Verlag, Grundlehr. der Math. Wiss. 319 (1999). MR1744486 (2000k:53038)

[BT1] M. Brin \& T. L. Thickstun, Open, irreducible 3-manifolds which are end 1-movable, Topology, 26, 211-233. MR0895574 (89f:57018)

[BT2] , 3-Manifolds which are end 1-movable, Mem. Amer. Math. Soc. 81 (1989), No. 411. MR0992161 (90g:57015)

[BB] J. Brock \& K. Bromberg, On the density of geometrically finite Kleinian groups, Acta Math. 192 (2004), no. 1, 33-93. MR2079598(2005e:57046)

[BBES] J. Brock, K. Bromberg, R. Evans \& J. Souto, Tameness on the boundary and Ahlfors' measure conjecture, Publ. Math. Inst. Hautes Études Sci. No. 98 (2003), 145-166. MR2031201 (2004k:30105)

[BCM] J. Brock, R. Canary \& Y. Minsky, The classification of Kleinian surface groups II: The ending lamination conjecture, eprint math.GT/0412006.

[BS] J. Brock \& J. Souto, Algebraic limits of geometrically finite manifolds are tame, preprint.

[BF] E. Brown \& C. D. Feustel, On properly embedding planes in arbitrary 3-manifolds, Proc. Amer. Math. Soc., 94, 173-178. MR0781077 (86e:57011)

[Caf] L. Caffarelli, Further regularity in the Signorini problem, Comm. in PDE 4 (1979), 10671076. MR0542512 (80i:35058) 
[Ca] R. Canary, Ends of hyperbolic 3-manifolds, J. Amer. Math. Soc. 6 (1993), 1-35. MR.1166330 (93e:57019)

[Ca2] A covering theorem for hyperbolic 3-manifolds and its applications, Topology, 35 (1996), 751-778. MR1396777 (97e:57012)

[CaM] R. Canary \& Y. N. Minsky, On limits of tame hyperbolic 3-manifolds and deformation theory of Kleinian groups, J. Diff. Geom. 43 (1996), 1-41. MR.1424418 (98f:57021)

[Car] L. Carleson, Selected problems on exceptional sets, Van Nostrand Mathematical Studies, 13, Princeton (1967). MR0225986 (37:1576)

[CLLM] A. Champanerkar, J. Lewis, M. Lipyanskiy \& S. Meltzer, Exceptional regions and associated exceptional hyperbolic 3-manifolds, preprint.

[CiSc] H. Choi \& R. Schoen, The space of minimal embeddings of a surface into a threedimensional manifold of positive Ricci curvature, Invent. Math. 81 (1985), 387-394. MR.0807063 (87a:58040)

[CM] T. Colding \& W. Minicozzi II, Minimal Surfaces, CIMS Lecture Notes 4 (1999). MR 1683966 (2002b:49072)

[EM] D. Epstein \& A. Marden, Convex hulls in hyperbolic space, a theorem of Sullivan, and measured pleated surfaces, London Math. Soc. Lecture Notes, 111 (1986), 113-254. MR 0903852 (89c:52014)

[Ev] R. Evans, Tameness persists in weakly type-preserving limits, Amer. J. Math. 126 (2004), no. 4, 713-737. MR2075479 (2005d:57023)

[Fed] H. Federer, Geometric measure theory, Springer-Verlag, Die Grundlehr. der math. Wiss. 153 (1969). MR0257325(41:1976)

[FF] B. Freedman \& M. Freedman, Kneser-Haken finiteness for bounded 3-manifolds, locally free groups, and cyclic covers, Topology 37, (1998), 133-147. MR1480882 (99h:57036)

[FHS] M. Freedman, J. Hass \& P. Scott, Least area incompressible surfaces in 3-manifolds, Invent. Math. 71 (1983), no. 3, 609-642. MR0695910 (85e:57012)

[Fre] J. Frehse, Two dimensional variational problems with thin obstacles, Math. Z. 143 (1975), 279-288. MR0380550(52:1450)

[G1] D. Gabai, Foliations and the topology of 3-manifolds, J. Diff. Geom. 18 (1983), 445-503. MR0723813 (86a:57009)

[G2] D. Gabai, On the geometric and topological rigidity of hyperbolic 3-manifolds, J. Amer. Math. Soc. 10 (1997), 37-74. MR1354958 (97h:57028)

[GMT] D. Gabai, R. Meyerhoff \& N. Thurston, Homotopy hyperbolic 3-manifolds are hyperbolic, Annals of Math. 157 (2003), 335-431. MR.1973051 (2004d:57020)

[Gr] M. Gromov, Metric structures for Riemannian and non-Riemannian spaces, Birkhäuser, Progress in Mathematics 152 (1999). MR1699320(2000d:53065)

[HS] J. Hass \& P. Scott, The existence of least area surfaces in 3-manifolds, Trans. Amer. Math. Soc. 310 (1988), 87-114. MR0965747 (90c:53022)

[Ha1] A. Hatcher, Homeomorphisms of sufficiently large $P^{2}$-irreducible 3-manifolds, Topology 15 (1976), 343-347. MR0420620 (54:8633)

[He] J. Hempel, 3-Manifolds Ann. of Math. Studies 86, Princeton University Press, Princeton, N. J. MR0415619 (54:3702)

[Jo] K. Johannson, Homotopy equivalences of 3-manifolds with boundaries, Springer LNM $\mathbf{7 6 1}$ (1979). MR0551744 (82c:57005)

[JR] K. N. Jones \& A. Reid, Vol3 and other exceptional hyperbolic 3-manifolds, Proc. Amer. Math. Soc. 129 (2001), 2175-2185. MR1825931 (2002e:57024)

[Js] J. Jost, Riemannian Geometry and Geometric Analysis, Springer-Verlag, Berlin; 3rd edition (2002) MR.1871261 (2002i:53001)

[K] D. Kinderlehrer, The smoothness of the solution of the boundary obstacle problem, J. Math. Pures et Appl. 60 (1981), 193-212. MR0620584 (84j:49011)

[KS] G. Kleineidam \& J. Souto, Ending laminations in the Masur domain, Kleinian Groups and Hyperbolic Manifolds, Proceedings, Cambridge University Press, 2003. MR2044547 (2005e:57048)

[Lew] H. Lewy, On the coincidence set in variational inequalities, J. Diff. Geom. 6 (1972) 497-501. MR0320343 (47:8882)

[Li] M. Lipyanskiy, A computer assisted application of Poincaré's fundamental polyhedron theorem, preprint. 
[Ma] A. Marden, The geometry of finitely generated Kleinian groups, Annals of Math. 99 (1974), 383-462. MR0349992 (50:2485)

[Mv] A.I. Malcev, On isomorphic matrix representations of infinite groups, Mat. Sb. (N. S.), 8 (50), (1940), 405-421. MR0003420 (2:216d)

[Mc] D. McCullough, Compact submanifolds of 3-manifolds with boundary, Quart. J. Math. Oxford Ser. (2), 37 (1986), 299-307. MR0854628 (88d:57012)

[MMS] D. McCullough, A. Miller \& G. A. Swarup, Uniqueness of cores of noncompact 3manifolds, J. London Math. Soc., 32 (1985), 548-556. MR0825931(87e:57018)

[MSY] W. Meeks, L. Simon \& S.-T. Yau, Embedded minimal surfaces, exotic spheres, and manifolds with positive Ricci curvature, Annals of Math. (2) 116 (1982), no. 3, 621-659. MR:0678484 (84f:53053)

[MY] W. Meeks \& S.-T. Yau, The classical Plateau problem and the topology of threedimensional manifolds, Topology 21 (1982), 409-442. MR0670745 (84g:53016)

[Me] G. R. Meyerhoff, A lower bound for the volume of hyperbolic 3-manifolds, Canadian J. of Math., 39 (1987), 1038-1056. MR0918586 (88k:57049)

[Mi] Y. Minsky, The classification of Kleinian surface groups I: Models and bounds, eprint math.GT/0302208.

[Mo] J. Morgan, On Thurston's uniformization for three-dimensional manifolds, The Smith Conjecture (New York 1979), Academic Press, Mathematics 112, 37-138. page 71. MR.0758464

[Mor] C. B. Morrey Jr., Multiple integrals in the calculus of variations, Springer, (1966). MR.0202511 (34:2380)

[Mw] G. Mostow, Quasiconformal mappings in n-space and the rigidity of hyperbolic space, Pubs. IHES 34 (1968), 53-104. MR0236383 (38:4679)

[My] R. Myers, End reductions, fundamental groups, and covering spaces of irreducible open 3-manifolds, Geom. Topol. 9 (2005), 971-990. MR2140996

[N] J. Nitsche, Variational problems with inequalities as boundary conditions or How to Fashion a Cheap Hat for Giacometti's Brother, Arch. Rational Mech. Anal. 35 (1969), 83-113. MR0248585 (40:1837)

[Oh] K. Ohshika, Kleinian groups which are limits of geometrically finite groups, Mem. Amer. Math. Soc. 177 (2005), no. 834. MR2154090

[Oss] R. Osserman, A survey of minimal surfaces, Second edition. Dover Publications (1986). MR0852409 (87j:53012)

[Re] Yu. Reshetnyak, Two-dimensional manifolds of bounded curvature, in Geometry IV, Encyclopaedia of Mathematical Sciences, vol. 70, Springer-Verlag (1989), 3-165. MR.1263964

[Ri] D. Richardson, Variational problems with thin obstacles, Thesis, UBC (1978).

[S] R. Schoen, Estimates for stable minimal surfaces in three dimensional manifolds, Annals of Math. Stud. 103, (1983), 111-126. MR0795231 (86j:53094)

[SY] R. Schoen and S.-T. Yau, Existence of incompressible minimal surfaces and the topology of three-dimensional manifolds with nonnegative scalar curvature, Ann. of Math. (2) 110, no. 1, (1979), 127-142. MR0541332 (81k:58029)

[Sc] G. P. Scott, Compact submanifolds of 3-manifolds, J. London Math. Soc. 7 (1973), 246250. MR 0326737 (48:5080)

[Som] T. Soma, Existence of polygonal wrappings in hyperbolic 3-manifolds, preprint.

[So] J. Souto, A note on the tameness of hyperbolic 3-manifolds, Topology, 44 (2005), no. 2, 459-474. MR 2114957 (2005m:57032)

[St] J. Stallings, On fibering certain 3-manifolds, Topology of 3-manifolds and related topics, Prentice-Hall, (1962), 95-100. MR0158375 (28:1600)

[T] W. P. Thurston, Geometry and topology of 3-manifolds, 1978, Princeton Math. Dept. Lecture Notes.

[T2] _ A norm for the homology of 3-manifolds, Mem. Amer. Math. Soc., 59 (1986), no. 339, 99-130. MR0823443 (88h:57014)

[Tu] T. Tucker, Non-compact 3-manifolds and the missing boundary problem, Topology, 13 (1974), 267-273. MR0353317 (50:5801)

[Tu2] - On the Fox-Artin sphere and surfaces in noncompact 3-manifolds, Quart. J. Math. Oxford Ser. (2), 28 (1977), 243-253. MR0448355 (56:6662) 
[Ve] I. N. Vekua, Systems of differential equations of the first order of elliptic type and boundary value problems, with an application to the theory of shells (Russian) Mat. Sbornik N. S. 31 (73) (1952), 217-314. MR0057444 (15:230a)

[Wa] F. Waldhausen, On irreducible 3-manifolds which are sufficiently large, Annals of Math. (2) 103 (1968), 56-88. MR0224099 (36:7146)

Department of Mathematics, Caltech, Pasadena, California 91125

Department of Mathematics, Princeton University, Princeton, New Jersey 08544 